\title{
THE IMPACTS OF SEX AND MYOGENIC CELL TRANSPLANTATION ON COLLATERAL
}

\section{CAPILLARY ARTERIOGENESIS}

\author{
A Thesis \\ presented to \\ the Faculty of California Polytechnic State University, \\ San Luis Obispo
}

In Partial Fulfillment

of the Requirements for the Degree

Master of Science in Biomedical Engineering

by

Padon Mary Sivesind

March 2018 
(C) 2018

Padon Mary Sivesind ALL RIGHTS RESERVED 


\section{COMMITEE MEMBERSHIP}

TITLE:

The Impacts of Sex and Myogenic Cell Transplantation on Collateral Capillary Arteriogenesis

AUTHOR:

DATE SUBMITTED:

COMMITTEE CHAIR:

COMMITTEE MEMBER:

COMMITTEE MEMBER:
Padon Mary Sivesind

March 2018

Trevor Cardinal, Ph.D.

Professor of Biomedical Engineering

Kristen Cardinal, Ph.D.

Professor of Biomedical Engineering

Lily Laiho, Ph.D.

Professor of Biomedical Engineering 


\title{
ABSTRACT
}

The Impacts of Sex and Myogenic Cell Transplantation on Collateral Capillary Arteriogenesis

\author{
Padon Mary Sivesind
}

Current treatments for peripheral arterial occlusive disease (PAOD) have limited success, so there is a need to develop more effective treatments. Because patients with native collaterals have a better prognosis, promoting collateral arteriogenesis is a potential PAOD treatment. Additionally, female PAOD patients have a worse response to treatment and a worse prognosis compared to males, which could be due to impaired collateralization. Cell transplantation is a potential treatment option to promote collateral arteriogenesis. Bone marrow derived stem cells are the main cell type that has been investigated, but they have had limited clinical success. Delivering a stem cell type native to the tissue like myogenic stem cells could have improved outcomes. In this study, the lateral spinotrapezius feed artery was ligated in male and female Balb/C mice to induce collateral capillary arteriogenesis, and 7 days post ligation arterialized collateral capillary (ACC) number and diameter were determined. There were no differences between sexes, which could be because young, healthy mice were used in this study rather than aged and diseased models. Because we observed no sex differences, we then assessed the effect of myogenic cell transplantation in male mice only. Immediately following ligation of the spinotrapezius feed artery, mice were treated with myogenic cells, thrombin, or vehicle, and 7 days post ligation ACC number and diameter were determined. Thrombin increased ACC number, but myogenic cells had no effect. However, myogenic cells increased ACC diameter, and both myogenic cells and thrombin decreased ACC number in the region of the muscle with the largest collateral, and increased the maximum ACC diameter. Another factor that could affect ACC formation is a pre-existing collateral (PEC), which only some $\mathrm{Balb} / \mathrm{C}$ mice have, so we also separated mice into PEC and non-PEC groups for analysis. In mice with a PEC, thrombin increased ACC number, and both myogenic cells and thrombin increased ACC diameter. There was a trend toward smaller arterialized capillaries in mice with a PEC, which could be because the majority of the blood flow is redirected through the PEC, so the PEC was the main vessel to remodel. These results are consistent with previous studies that indicated that thrombin augments arteriogenesis as well as increasing V-CAM, and suggest that myogenic cells have a similar effect possibly by secreting arteriogenic factors such as VEGF and MMPs. Because myogenic cells increase arteriogenesis, and macrophages are an essential regulator of arteriogenesis, we also tested the hypothesis that myogenic cells would increase macrophage content. Macrophage number increased with ligation, but there was no difference in macrophage number between any of the treatment groups. The lack of difference in macrophage number could be because the day 7 timepoint was too late, as macrophage content peaks at day 3. Because myoblasts increased arteriogenesis, they also may have increased the number M2 macrophages, which are the main macrophage contributor to arteriogenesis, but we used a general macrophage marker and could not detect an increase in M2 macrophages. In future studies, to determine if there is an increase in M2 macrophages a stain specific to M2 macrophages like CD206 could be added. Additionally, a diabetic Balb/C strain could be used to determine if arteriogenesis is impaired in males compared to females in a diseased model.

Keywords: collateral capillary arteriogenesis, sexual dimorphism, cell transplantation 


\section{ACKNOWLEDGMENTS}

I would like to thank Dr. Cardinal for giving me the opportunity to participate in research and for his support and guidance throughout this project.

I would also like to thank my parents for being my cheerleaders, and my Aunt Ella, Uncle Matt, and my cousin's Jane and Posey for welcoming me into their home and keeping me entertained while I was writing this.

Finally, thanks the members of the MAVR lab for all your support and friendship.

"Just because we don't understand doesn't mean the explanation doesn't exist" -Madeleine L'Engle 


\section{TABLE OF CONTENTS}

Page

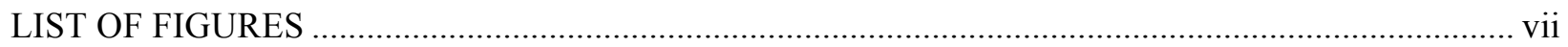

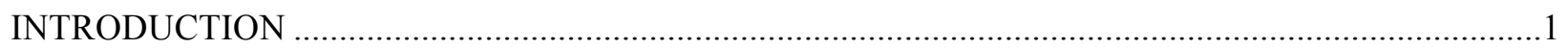

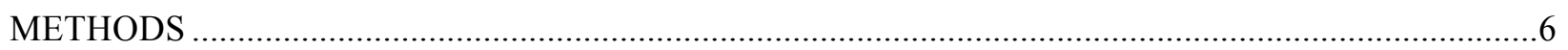

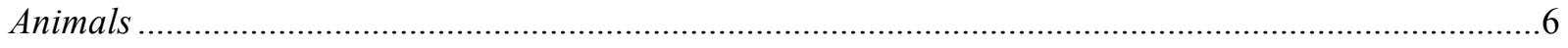

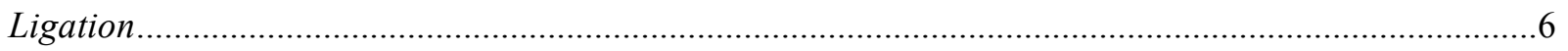

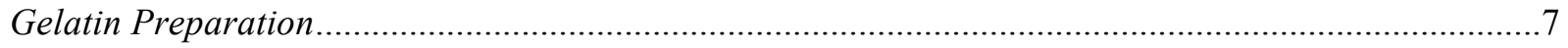

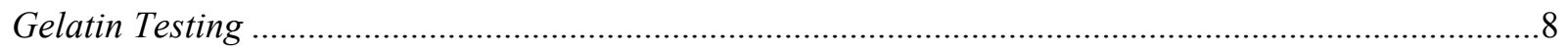

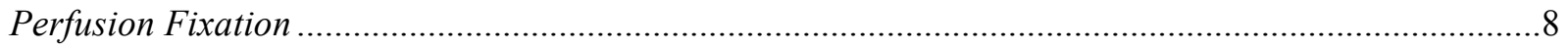

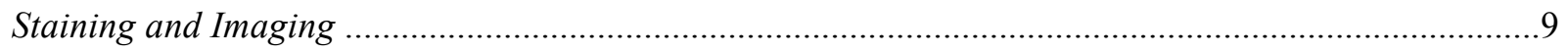

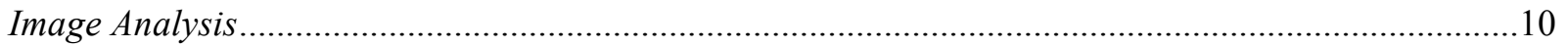

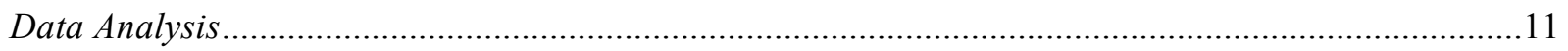

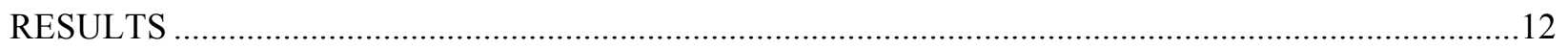

Female versus Male Collateral Capillary Arteriogenesis.............................................................12

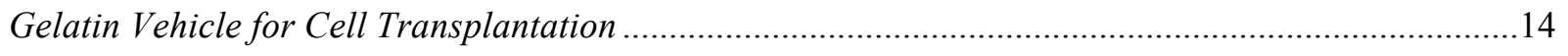

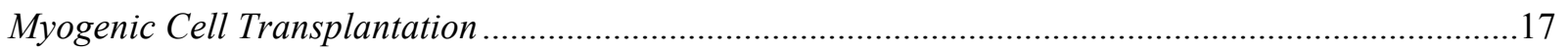

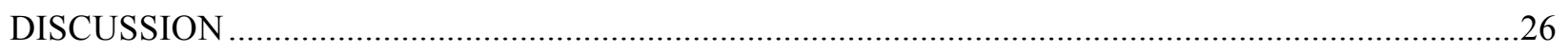

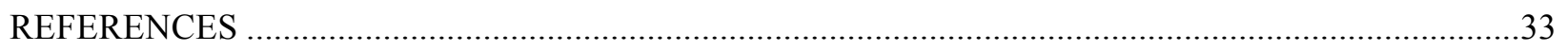

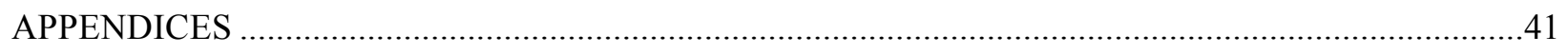

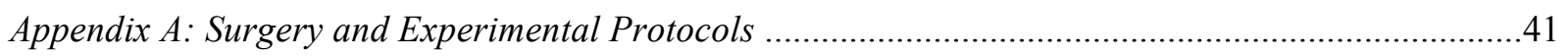

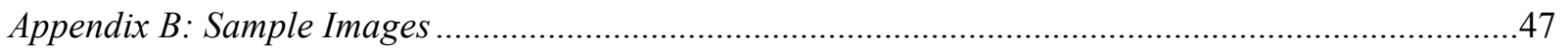

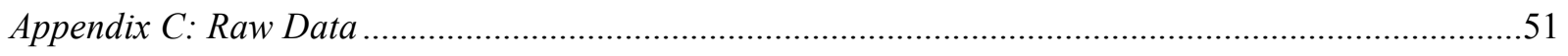

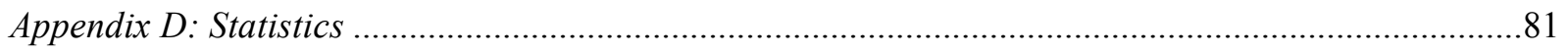




\section{LIST OF FIGURES}

Figure 1. Current Treatments for PAOD .......

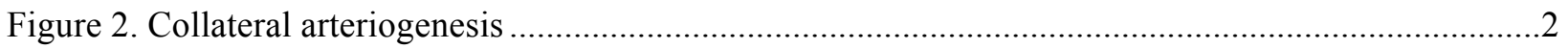

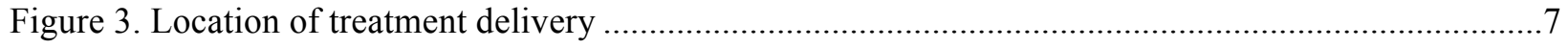

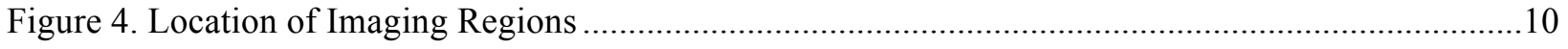

Figure 5. Confocal images of arterialized collateral capillaries ..........................................................12

Figure 6. No difference in number of alpha-smooth muscle positive vessels between males and

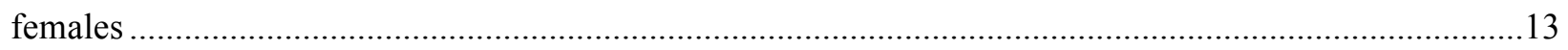

Figure 7. No difference between males and females in arterialized collateral capillary diameter ..............14

Figure 8. Cross-linked gelatin vehicle remained polymerized for 7 days in vivo ......................................15

Figure 9. Fibroblasts have normal morphology after one day of culture on gelatin vehicle ......................16

Figure 10. Confocal images of arterialized collateral capillary region....................................................17

Figure 11. Thrombin increases number of arterialized collateral capillaries............................................18

Figure 12. Thrombin decreases number of arterialized collateral capillaries in region with largest

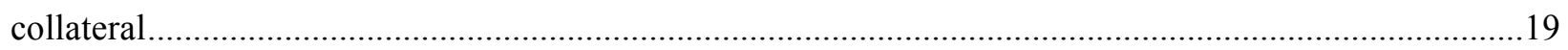

Figure 13. Myogenic cells increase arterialized collateral capillary size ...............................................20

Figure 14. Myogenic cells increase maximum arterialized collateral capillary diameter .........................21

Figure 15. Thrombin increases number of arterialized collateral capillaries in mice without PECs..........22

Figure 16. Myogenic cells increase arterialized collateral capillary diameter in mice without PECs.........23

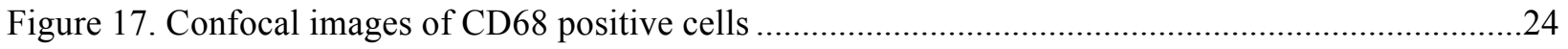

Figure 18. Myogenic cell transplantation has no effect on CD68 cell count...........................................25

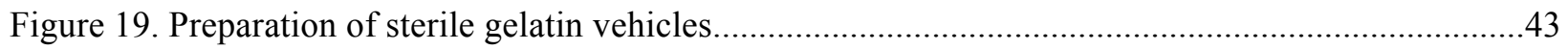




\section{INTRODUCTION}

Peripheral arterial occlusive disease (PAOD) affects 8 to 12 million people in the United States and can lead to critical limb ischemia (CLI) and amputation [1]. Current treatments include stenting and bypass grafting (Figure 1), but only $50 \%$ of CLI patients are eligible, and after 1 year, only $25 \%$ of revascularization attempts in these patients are successful [2]. Amputation is the only option for ineligible patients, and within 6 months, about $40 \%$ of ineligible patients have a limb amputated and $20 \%$ die [2]. The insufficiency of current treatments suggests that promoting collateral arteriogenesis may be a desirable treatment option. Collaterals are anastomoses between two arterioles that provide an alternative route for blood flow to bypass an arterial occlusion. Patients with pre-existing collaterals in their coronary circulation have better myocardial viability, a reduced risk for a repeat cardiac event, and a lower hospital mortality rate following heart attack or revascularization $[3,4,5]$. In the peripheral circulation, a welldeveloped collateral circulation improves 6 minute walk test results, and slows performance decline over time [6,7]. Unfortunately, not all humans have a native collateral network, and about $30 \%$ of patients never develop angiographically-identifiable collaterals $[8,9]$.
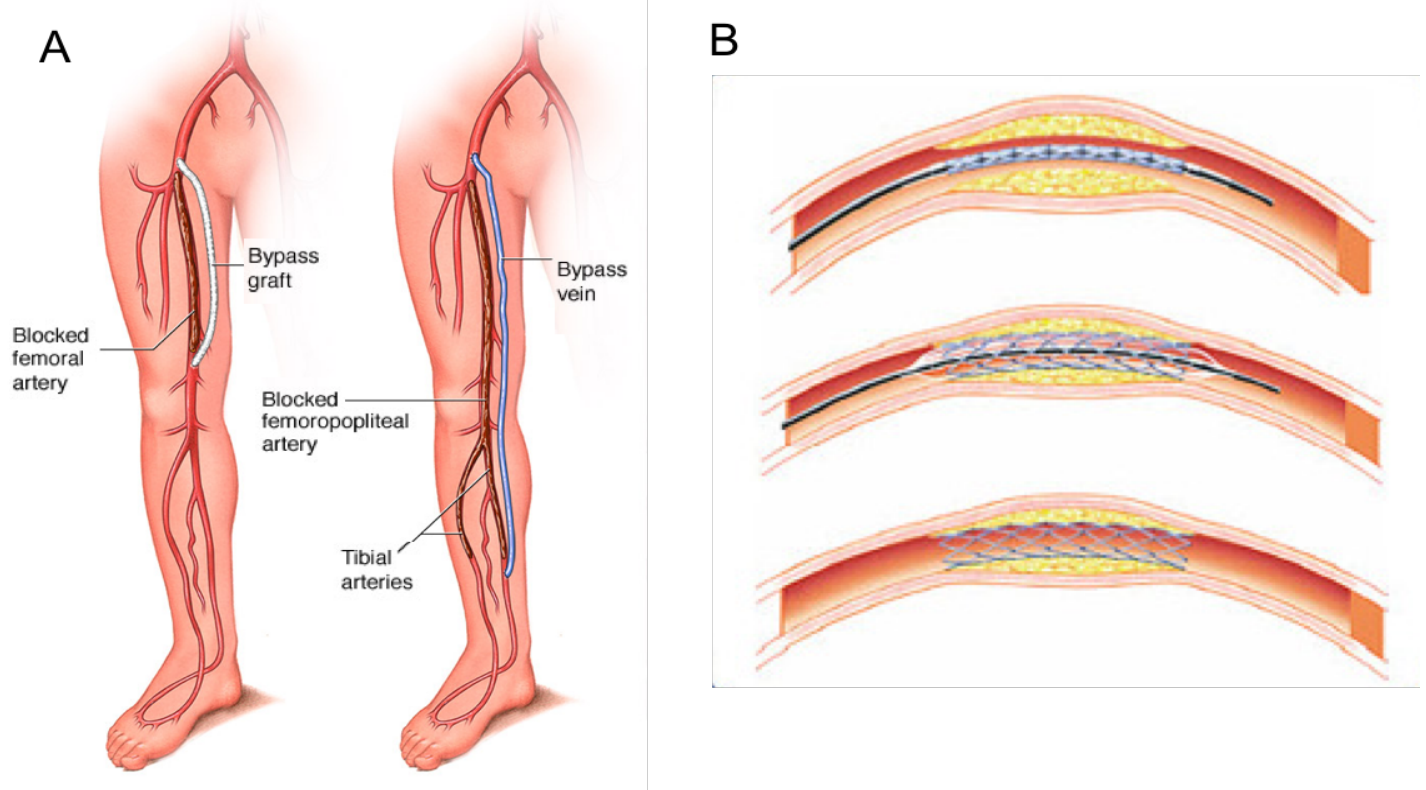

Figure 1. Current Treatments for PAOD. A) Both bypass grafting (left) and inserting a bypass vein (right) are common treatments for PAD to allow bloodflow around the occlusion. Adapted from [10] B) Stenting is also used to reopen the occluded artery. Adapted from [11] 
Similar to humans, animals also have varying degrees of collateral formation in response to an occlusion. For example, mice from the $\mathrm{C} 57 \mathrm{Bl} / 6$ strain have many pre-existing collaterals that readily enlarge, while mice from the Balb/C strain have fewer pre-existing collaterals that have impaired enlargement [12]. When pre-existing collaterals are present, an occlusion causes the pressure gradient to increase across the collateral, which elevates blood flow, increases the shear stress, activates endothelial cells, and leads to macrophage recruitment $[13,14]$. Macrophages secrete matrix metalloproteinases to degrade the matrix, while endothelial cells and smooth muscle cells proliferate in response to growth factors secreted by macrophages and expand the arterial diameter until the shear stress is normalized (Figure 2) $[13,14]$. In tissues of Balb/C mice without pre-existing arteriolar collaterals, an arterial occlusion causes arterialization in capillaries that connect ischemic an non-ischemic terminal arterioles [15]. These collateral capillaries outwardly remodel and gain a smooth muscle cell layer either by recruiting smooth muscle cells from an upstream vessel or through perivascular cell differentiation [15].

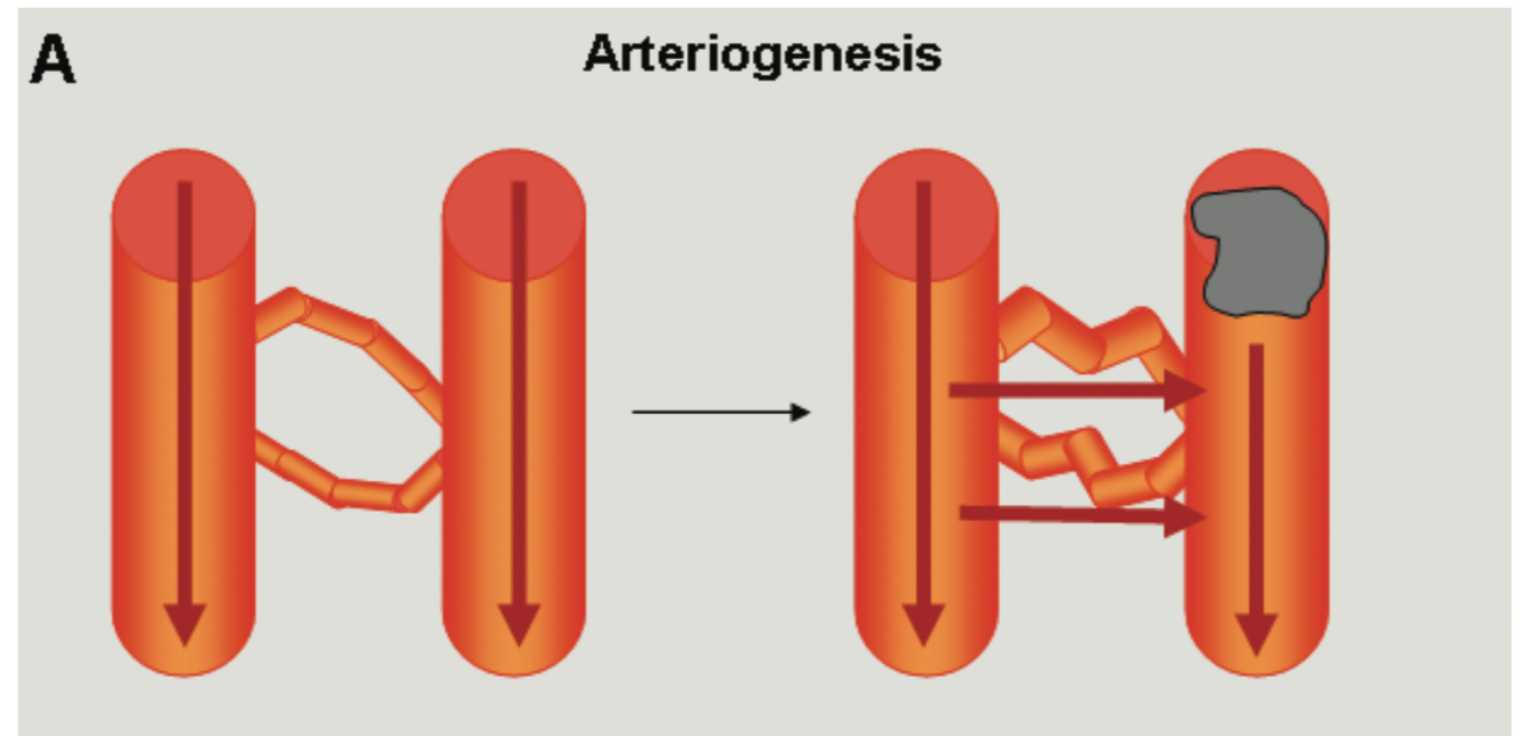

Figure 2. Collateral arteriogenesis. Before an occlusion, blood flows through both arterioles and there is a limited pressure gradient through the collaterals (left). Following an occlusion (grey), blood flow is blocked in one of the arterioles, and the pressure gradient in the collaterals increases, which leads to elevated blood flow and increased shear stress and remodeling in the collateral vessels (right). Adapted from Heil et al [16].

The first approach to target arteriogenesis as a treatment for PAOD involved delivering single factors known to contribute to arteriogenesis, which were injected either intramuscularly in the limb 
requiring treatment or intravenously in a major vein $[17,18]$. The factors investigated included VEGF, FGF and HGF as both gene and protein therapy, and the transcription factor HIF-1 $\alpha$ as gene therapy $[17,18,19,20,21]$. In early clinical studies and animal models, VEGF, FGF, HIF-1 $\alpha$ and HGF effectively increased perfusion, ulcer healing, and limb salvage $[17,18,19,20,21]$. However, when these factors were tested in larger blinded, randomized controlled trials, the treatments had no effect on end points such as ankle-brachial index, rest pain, exercise performance, or ulcer healing $[22,23,24,25,26]$.

The failure of single factor treatments led to the investigation of cell transplantation to stimulate arteriogenesis as a treatment for PAOD. Because arteriogenesis signaling involves multiple growth factors, delivering cells as a treatment may be more effective than delivering a single growth factor $[27,28]$. The first cell type investigated was bone marrow mononuclear cells (MNCs). In early clinical trials, intramuscular injections of bone marrow MNCs increased ulcer healing and amputation free survival, but had no effect on limb perfusion, pain free walking distance or ankle-brachial index $[29,30,31]$. These results were positive enough to perform larger clinical trials, but in randomized controlled trials, bone marrow MNCs had no effect on amputation rate, ankle-brachial index or ulcer healing $[32,33,34]$. Another limitation with bone marrow MNCs is that they cannot be cultured once isolated, and therefore a large volume of bone marrow must be obtained [35]. However, another bone marrow derived cell, bone marrow mesenchymal stem cells (MSCs), requires significantly less bone marrow because the cells remain potent when expanded after isolation [36]. Bone marrow MSCs also increased perfusion in the rat hindlimb more than bone marrow MNCs [37], but similar to bone marrow MNCs, they have had mixed clinical outcomes. When injected into the heart, bone marrow MSCs had no effect on left ventricle function at 18 months [38]. Additionally, intramuscular injections of bone marrow MSCs lead to a trend towards reduced amputation rates and rest pain, but had no effect on ankle brachial index $[39,40]$. Even though bone marrow cells have shown some promise in the clinic, they must be isolated through a bone marrow biopsy which can be painful for the patient and sometimes requires general anesthesia [41,42]. Isolation of endothelial progenitor cells (EPCs), another potential treatment for PAOD, is less painful than a bone marrow biopsy. EPCs can be isolated from the peripheral blood 
after dosing with granulocyte colony stimulating factor (G-CSF), which stimulates EPCs migration into the blood [43]. In non-placebo controlled clinical trials, intramuscular injections of EPCs increased partial oxygen pressure and pain free walking distance and decreased ulcer size, but there was no difference in ankle brachial index $[43,44]$. When a placebo control was used, there was a trend towards a lower amputation rate at 6 and 12 months but no difference in ankle brachial index, pain free walking distance, or wound healing [45].

Although bone marrow-derived cells do not appear to be a viable alternative to revascularization treatments, exercise is already a successful PAOD treatment. Exercise treatment is as good or better than revascularization as a treatment for PAOD [46,47], and regimes that include eccentric contractions had increased walking ability [48]. However, exercise treatment is only effective when performed under supervision $[49,50]$. Therefore, a potential treatment for PAOD is delivering a cell type that is activated during eccentric exercise such as satellite cells [51]. Satellite cells recruit monocytes in response to muscle injury [52,53], and macrophages are more efficient at paracrine signaling in muscle tissue when satellite cells are present [54]. Satellite cells also have angiogenic properties [55,56], but their effect on arteriogenesis has not been investigated. Additionally, tissue resident stem cells may have an advantage over other cell types because they may be able to communicate within the 'myo-vascular' niche more effectively than non-tissue resident stem cells. For example, tissue resident mesenchymal stem cells more effectively differentiate into muscle and cartilage than bone marrow derived mesenchymal stem cells $[57,58,59]$. The stem cell niche is important in maintaining stem cell properties including how they communicate with surrounding cells $[60,61]$. Therefore, bone marrow-derived cells, which have been removed from their niche, may communicate less effectively within the 'myo-vascular' niche than tissue resident stem cells, which could potentially contribute to their reduced efficacy as a revascularization treatment. Another advantage of muscle derived stem cells is that the isolation only requires a muscle biopsy $[62,63]$ rather than additional treatment with G-CSF that is required to isolate EPCs or a bone marrow biopsy that is required to isolated bone marrow MSCs and MNCs. 
To develop effective new PAOD treatments, differences in efficacy of current treatments must be understood so new treatments do not have the same weaknesses. For example, women do not seem to respond to treatment as well as men. There is a higher incidence of wound complications after bypass grafting in women [64], a higher rate of hospital mortality [65], and less positive results from exercise treatment than in males [66]. Additionally, females with PAOD appear to have a worse prognosis than males, with a shorter walking distance [67], and a higher rate of critical limb ischemia [68]. The sexual dimorphism seen in humans could be explained through investigation of processes related to PAOD in animal models. For example, bone marrow $\mathrm{MNCs}$ reduce aortic plaque in male, but not female, $\mathrm{ApoE}^{-/-}$ knockout mice [69]. Impaired collateralization could be another cause of the clinical difference between males and females. In patients with an occluded coronary artery, females were less likely than males to have an angiographically identifiable collateral [70], suggesting that arteriogenesis might be impaired in females. Since the number of female patients with PAOD is at least equal to the number of males [71], and the number of women with the PAOD is expected to increase [72], it is important to understand any sex differences so women with PAOD can be treated effectively.

In summary, clinically, females appear to have a worse prognosis and response to treatment than males, which could be due to impaired collateralization in females. Additionally, myogenic stem cells have angiogenic properties [55,56], but their effect on arteriogenesis is unknown. Therefore, we tested the hypotheses that collateral capillary arteriogenesis is impaired in females compared to males, and that myogenic cell transplantation would enhance collateral capillary arteriogenesis and macrophage recruitment. To support the second hypothesis, we developed a gelatin hydrogel to use as a vehicle for myogenic cell transplantation. 


\section{METHODS}

Animals

Male and Female Balb/C mice (Taconic Farms; Oxnard, CA and Jackson Labs; Sacramento, CA) between 8 and 14 weeks, and Male ICR mice (Cal Poly Vivarium) were housed 2 to 4 mice per microisolator cage with ad libetum access to food and water, plus enrichment (a mouse 'house' and a plastic tube). The cages were in a temperature controlled room with a 12-hour light/dark cycle. The male ICR mice were used during gelatin vehicle testing; $\mathrm{Balb} / \mathrm{C}$ mice were used for all other experiments. To determine if collateral capillary arteriogenesis is impaired in females, mice were divided into male $(n=8)$ and female ( $n=8)$ groups. For the myogenic cell transplantation study, only male mice were used. Mice were divided into 4 groups: myogenic cells $(n=6)$, vehicle $(n=6)$, thrombin $(n=6)$, and ligation only $(n=6)$. All procedures were approved by the California Polytechnic University Institutional Animal Care and Use Committee.

\section{Ligation}

Animals were anesthetized in an induction chamber with 5\% isofluorane in oxygen flowing at 3 $1 \cdot \min ^{-1}$. After anesthetization, isofluorane was reduced to $1-3 \%$ flowing at $0.5-1.01 \cdot \mathrm{min}^{-1}$. Prior to surgery, trimming clippers and depilatory cream were used to remove hair from the dorsal area of the mouse, which was then disinfected with chlorohexidine diacetate (Nolvasan). Animals were maintained at $35^{\circ} \mathrm{C}$ during surgery with a heat pad and a rectal temperature probe, and ophthalmic ointment was applied to the cornea to prevent desiccation. A small incision was made parallel to the spine over the lateral edge of the spinotrapezius. The fat pad overlying the spinotrapezius was undermined using blunt dissection to expose the fat pad underlying the lateral edge of the muscle that contains the feed artery/vein pair. The artery was separated from the vein, and two single strands from 6-0 silk suture were passed under the artery. The sutures were tied around the artery in two locations, and the artery was transected between the two sutures before closing the incision using 7-0 prolene suture. On the sham side, the same procedure was repeated up to the separation of the artery from the vein. 
In animals receiving either myogenic cells or vehicle, the spinotrapezius was undermined by gentle blunt dissection to create a pocket for placing the cell construct or the vehicle (Figure 1). A cylinder of the cell construct was removed with a $1.5 \mathrm{~mm}$ biopsy punch and inserted into the pocket. For mice receiving thrombin, $1 \mu \mathrm{L} \cdot \mathrm{g}^{-1}$ body weight of thrombin $(1 \mathrm{NIH}$ unit/ $\mu \mathrm{L})$ was superfused superficially and injected deep to the muscle following ligation (Figure 3).

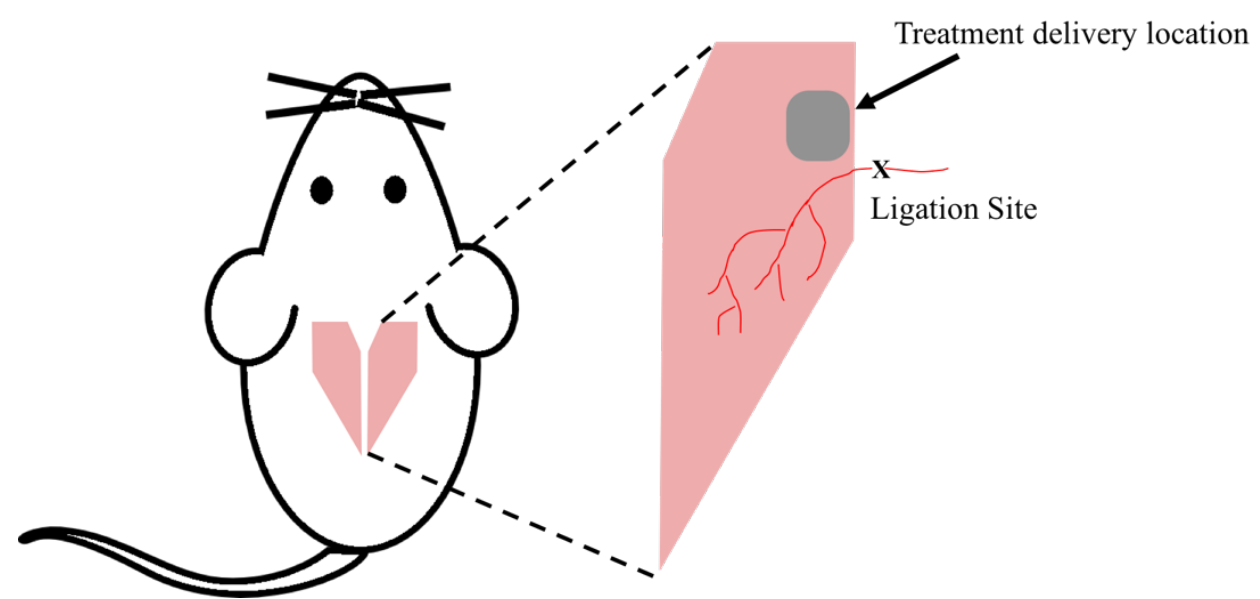

Figure 3. Location of treatment delivery. Following ligation of the feed artery, the spinotrapezius was undermined in the location shown (black arrow) by gentle blunt dissection to create pocket for placing the cell construct or the vehicle. For thrombin treatment delivery, $1 \mathrm{uL} \cdot \mathrm{g}^{-1}$ body weight of thrombin $(1 \mathrm{NIH}$ unit $/ \mu \mathrm{L})$ was superfused above and injected below the muscle in the location shown (grey area).

\section{Gelatin Preparation}

A $10 \%$ gelatin solution was prepared aseptically in an Erlenmeyer flask in a biological safety cabinet. On a hot plate outside the biological safety cabinet, the solution was boiled for 5 minutes to sterilize and solubilize the gelatin. After boiling, the gelatin solution was transferred to a 24 well plate and stored at room temperature until it polymerized. After polymerization, the gelatin was cross-linked with 6mM 1-Ethyl-3-(3-dimethylaminopropyl) carbodiimide (EDC) and stored overnight at $4{ }^{\circ} \mathrm{C}$. After storage, the gels were washed 3 times for 30 minutes with PBS.

Myoblasts isolated from Balb/C mice were dissociated with a Cell Dissociation Buffer (EDTA, ThermoFisher, Waltham, MA), centrifuged at $300 \mathrm{~g}$ for 5 minutes, suspended in growth media (20\% FBS, $2 \mu \mathrm{l} / \mathrm{ml}$ media bFGF (1 $\mu \mathrm{g} / \mathrm{ml}), 2 \mu \mathrm{l} / \mathrm{ml}$ media SB $203580(10 \mathrm{mmol})$ in Hams F-10 Nutrient Mixture with 
$1 \%$ Pen/Strep), and 100,000 cells were sodded onto one well of gelatin so that approximately $9.6 \times 10^{3}$ cells would be transplanted in the $1.5 \mathrm{~mm}$ diameter punch. Gelatin vehicles were stored in the incubator overnight to allow for cell adhesion.

\section{Gelatin Testing}

Both $4 \%$ and $10 \%$ gelatin vehicles were prepared aseptically in a $24-w e l l$ plate. After polymerization, vehicles were cross-linked with either $6 \mathrm{mM}$ or $12 \mathrm{mM}$ EDC. Gelatin vehicles without cross-linker were also prepared through a similar procedure, without the addition of EDC. The vehicles were incubated overnight at $4{ }^{\circ} \mathrm{C}$ to allow for crosslinking and washed 3 times for 30 minutes with phosphate buffered saline (PBS) after storage. For acute testing, vehicles were either implanted deep to the spinotrapezius or stored in the incubator overnight to determine which vehicle types remained polymerized at $37^{\circ} \mathrm{C}$. To test the cytotoxicity of cross-linked gelatin, $3 \mathrm{~T} 3$ fibroblasts were sodded onto each vehicle type. Cell morphology was observed 1, 3, and 12 hours after sodding. To test vehicle retention, both $4 \%$ and $10 \%$ vehicles cross-linked with $6 \mathrm{mM}$ EDC were implanted deep to the spinotrapezius ( $\mathrm{n}=2$ ). After seven days, the spinotrapezius was resected to determine if the vehicle remained in place.

\section{Perfusion Fixation}

At 7 days following ligation, mice were perfusion fixed with paraformaldehyde in preparation for immunofluorescence. Prior to fixation, overlying fascia was removed from the spinotrapezius muscles. Through a thoracotomy, a catheter was placed in the left ventricle, and $40 \mathrm{~mL}$ of vasodilator solution $\left(10^{-3}\right.$ SNP, $10^{-4}$ adenosine, and $1 \mathrm{U} / \mathrm{ml}$ heparin in PBS) was perfused through the circulation at $2 \mathrm{ml} \cdot \mathrm{min}^{-1}$ using a syringe pump to displace the blood and maximally dilate the vasculature. Then $5 \mathrm{~mL}$ of $4 \%$

paraformaldehyde (PFA) was perfused at $1 \mathrm{ml} \cdot \mathrm{min}^{-1}$ to fix the tissue. Following fixation, spinotrapezius muscles were undermined, resected, and underlying fascia was removed. Muscles were post-fixed overnight in $4 \%$ PFA at $4{ }^{\circ} \mathrm{C}$ and then transferred to PBS and stored at $4{ }^{\circ} \mathrm{C}$ until staining. 


\section{Staining and Imaging}

To determine arterialized collateral capillary (ACC) diameter, number, and smooth muscle cell coverage, the spinotrapezius muscles were stained with 1:200 1A4 clone alpha-smooth muscle actin (smooth muscle cells, Cy3, Sigma-Aldrich 1mg/mL) and 1:20 Isolectin GS-IB4 from GRI (endothelial cells, Alexa Fluor 647, Thermo Fisher Scientific $1 \mathrm{mg} / \mathrm{mL}$ ). To determine macrophage number, spinotrapezious muscles were stained with 1:100 CD68 FA-11 Clone (macrophages, Alexa Fluor 488, Bio-Rad $0.05 \mathrm{mg} / \mathrm{mL}$ ), which is a lysosomal/endosomal-associated membrane glycoprotein that is expressed on lysosomes and endosomes [73]. All antibodies were prepared in PBS with $0.1 \%$ Triton X100 and $2 \%$ BSA, and incubated at $4{ }^{\circ} \mathrm{C}$ for 72 hours. The muscles were then washed 3 times for 20 minutes with $0.1 \%$ Triton X-100 in PBS, and then for 30 minutes in PBS. Muscles were mounted on glass slides in a 50:50 PBS:glycerol solution.

Using a 20x objective on a confocal microscope (Olympus FV 1000, Olympus, Center Valley, Pennsylvania), three regions were imaged between ischemic and perfused arteriolar trees, where ACCs are located in operated muscle (Figure 4). To find the appropriate imaging area, the ischemic arteriolar tree was followed down to a terminal arteriole where ACCs were identified because they are similar in appearance to capillaries, but they are also surrounded by smooth muscle cells. Unlike typical capillaries, ACCs connect two terminal arterioles, are relatively straight, and their long axis is parallel to the muscle fibers. On the sham side, the appropriate imaging location was found by following the arteriolar tree that would be the ischemic tree in the operated muscle to a terminal arteriole until there was also a terminal arteriole from a different arteriolar tree in the field of view. At each location, a $150 \mu \mathrm{m}$ thick z-stack with $5 \mu \mathrm{m}$ thick slices was obtained. 


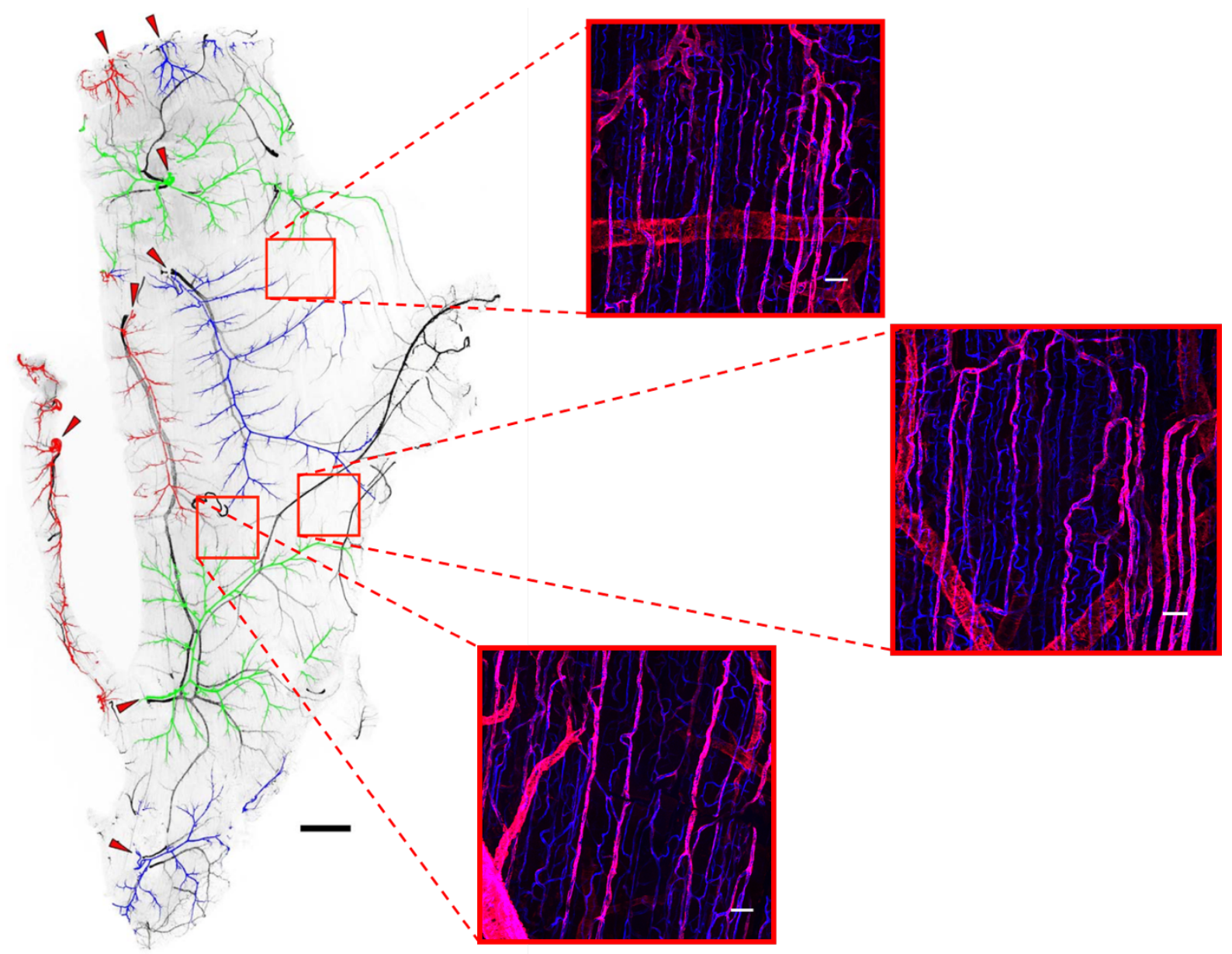

Figure 4. Location of Imaging Regions. Each spinotrapezius muscle was imaged using a 20x objective on a confocal microscope. The muscles were imaged at three locations between arteriolar trees (red squares) where ACCs are located on ligated muscles. Red staining is alpha-smooth muscle actin. Blue staining is lectin. Scale bar on montage is $1 \mathrm{~mm}$. Scale bars on regional images are $50 \mu \mathrm{m}$. Figure adapted from Mac Gabhann et al [15].

\section{Image Analysis}

To determine the number and diameter of capillaries and ACCs, the z-stacks were compressed and the channels were separated using ImageJ software. Alpha-smooth muscle positive vessels (ACCs) and lectin positive vessels (capillaries) were counted in a $200 \mu \mathrm{m}^{2}$ region in the compressed image. Inner diameters of three alpha-smooth muscle positive vessels and lectin positive vessels were also measured in each region.

To determine the maximum ACC diameter, the diameter of the largest collateral was measured in each replicate. To determine ACC number in the region of the largest collateral, all alpha-smooth muscle 
actin positive vessels were counted along a line drawn perpendicular to the ACCs in the region with the largest collateral.

CD68 positive cells were counted by finding large oval or elliptical areas of that were oriented around an ACC. To determine overall macrophage number, CD68 positive cells were counted within the same $200 \mu \mathrm{m}^{2}$ region as the ACCs. To determine vessel associated macrophage number, CD68 positive cells that were within $50 \mu \mathrm{m}^{2}$ of an ACC were counted along the entire length of the ACC.

\section{Data Analysis}

Differences in vessel number and diameter in males and females and in ligated and sham muscles were determined by independent t-tests. Differences in number and diameter of vessels in the four groups (myogenic cells, vehicle, thrombin, and ligation only) and in ligated and sham muscles were determined using One-Way ANOVA.

Differences in maximum diameter and ACC number in the four groups (myogenic cells, vehicle, thrombin, and ligation only) and in ligated and sham muscles were determined using One-Way ANOVA. Differences in number of CD68 positive cells in the four groups (myogenic cells, vehicle, thrombin, and ligation only) and in ligated and sham muscles were determined using One-Way ANOVA. Data are presented as mean $\pm \mathrm{SE}$. 


\section{RESULTS}

Female versus Male Collateral Capillary Arteriogenesis

To test the hypothesis that collateral capillary arteriogenesis is impaired in female mice as compared to male mice, we used confocal microscopy (Figure 5) to count and measure the diameters of collateral capillaries and arterialized collateral capillaries (ACCs) that connect ischemic and perfused arteriolar trees.

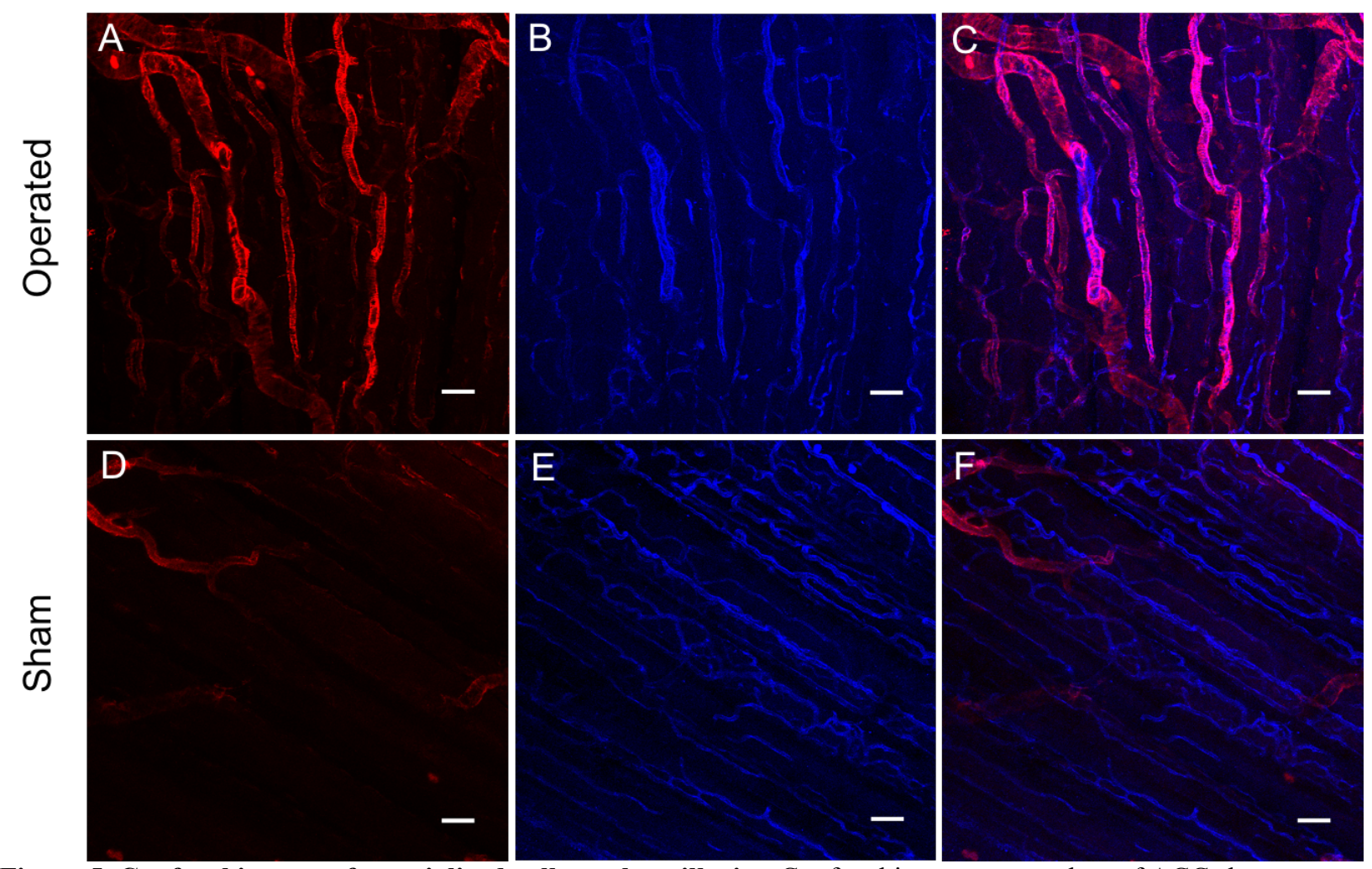

Figure 5. Confocal images of arterialized collateral capillaries. Confocal images were taken of ACCs between the ligated arteriolar tree and an adjacent, perfused arteriolar tree using a 20x objective. (A,D) Alpha-smooth muscle actin positive vessels show arterioles and ACCs. (B,E) Lectin positive vessels show both capillaries and ACCs. $(\mathrm{C}, \mathrm{F}) \mathrm{A}$ composite image combining $\mathrm{A}$ and $\mathrm{B}$ or $\mathrm{E}$ and $\mathrm{F}$. A-C are from an operated muscle and D-F are from a sham muscle. Scale bars are $50 \mu \mathrm{m}$.

There was no difference in the overall number of vessels between male and female mice or between ligated and sham muscles $(10.8 \pm 0.4$ female operated, $11.2 \pm 0.3$ female sham, $11.6 \pm 0.6$ male operated, $11.8 \pm 0.5$ male sham). To determine the number of ACCs, we counted the number of alphasmooth muscle actin positive vessels in the capillary position. As expected, the number of alpha-smooth muscle actin positive vessels increased in operated muscles (zero for both male and female sham, $4.6 \pm$ 
0.1 female operated, $4.9 \pm 0.1$ male operated). Additionally, there was a trend $(p=0.08)$ towards an increase in presumptive ACCs in males (Figure 6), but qualitatively it did not seem like there was a difference between males and females. There was also limited variation in ACC number between imaging regions, which suggests that 3 regions were sufficient for imaging.

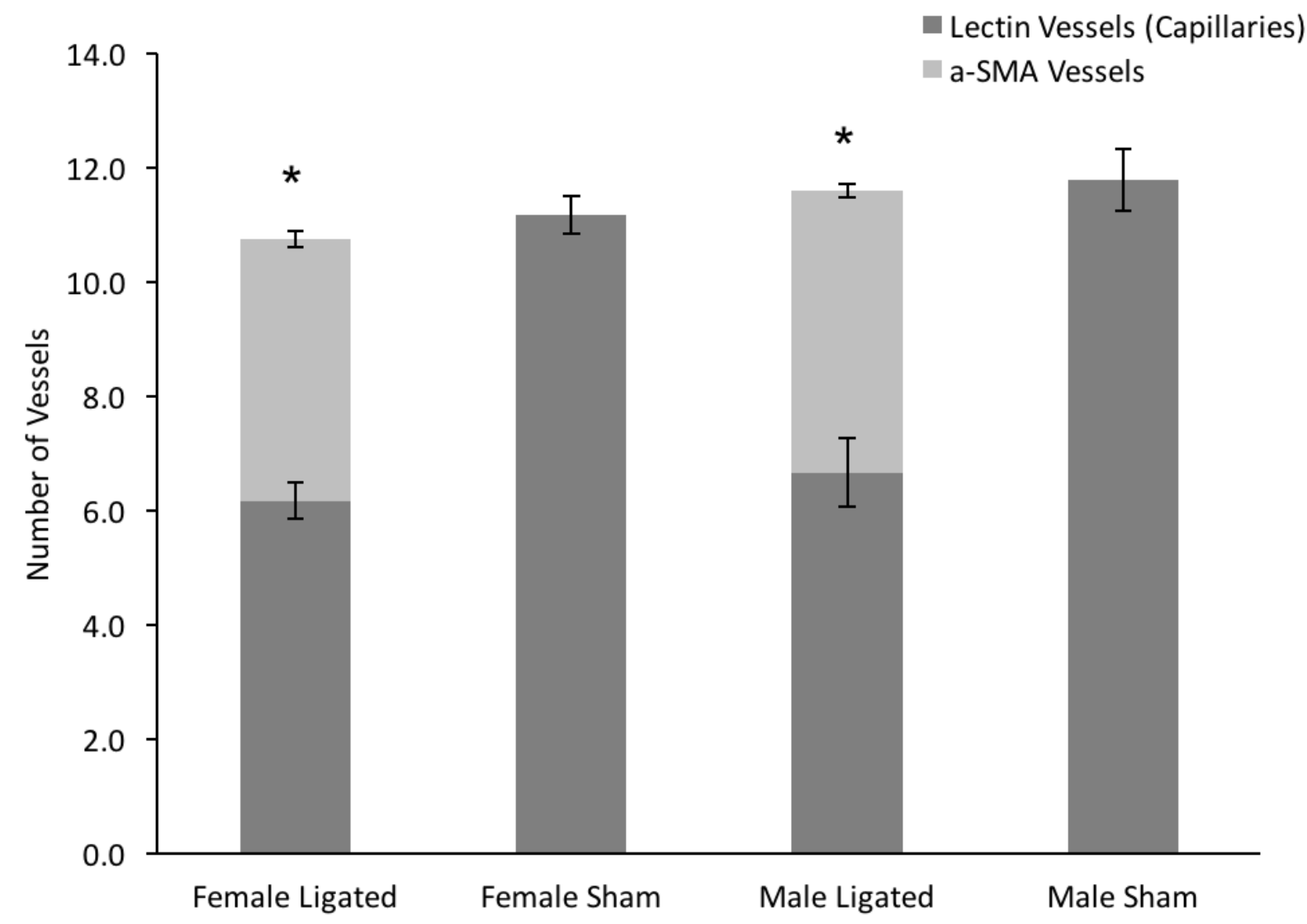

Figure 6. No difference in number of alpha-smooth muscle positive vessels between males and females.

Capillaries and ACCs were counted between ischemic and perfused arteriolar trees on both male $(\mathrm{n}=6)$ and female $(\mathrm{n}=5)$ replicates at day 7 using a 20x objective on a confocal microscope. The number of alpha-smooth muscle actin positive vessels increased in operated muscles. There was no difference between males and females in the number of ACCs. There was also no difference between males and females or ligated and sham in capillary number. $*^{*}, \mathrm{p}<0.05$ for operated versus sham)

As expected, ACCs had a larger diameter $(10.8 \pm 0.5 \mu \mathrm{m}$ in females and $11.2 \pm 0.3 \mu \mathrm{m}$ in males $)$ than capillaries $(4.7 \pm 0.2 \mu \mathrm{m}$ in females and $4.6 \pm 0.1 \mu \mathrm{m}$ in males), indicating outward remodeling occurred, but there was no difference in male and female ACC diameter or in capillary diameter between 
ligated and sham muscles (Figure 7). There was also limited variation in ACC diameter between imaging regions, which suggests that 3 regions were sufficient for imaging.

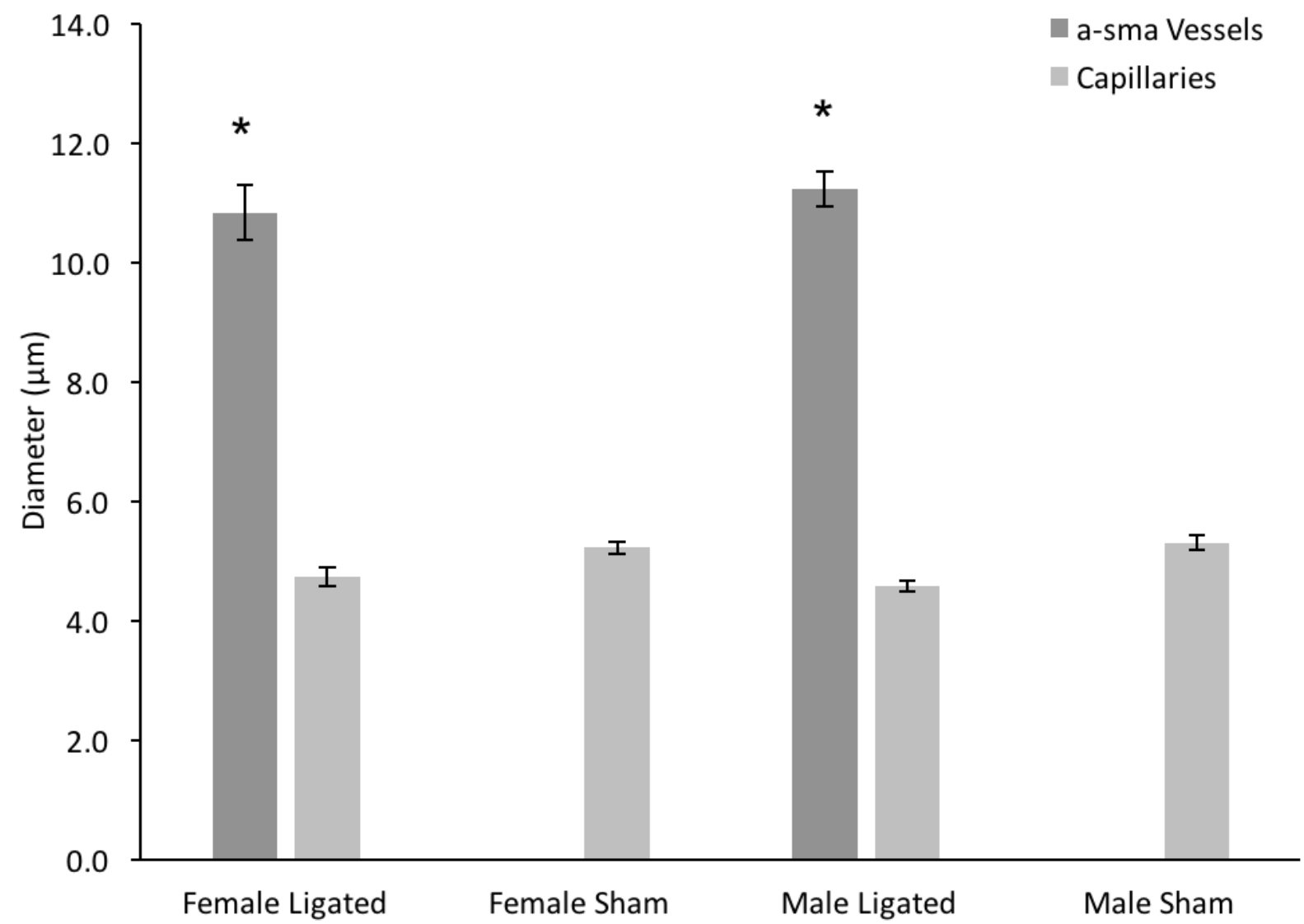

Figure 7. No difference between males and females in arterialized collateral capillary diameter. Capillary diameter and ACC diameter were measured between ischemic and perfused trees on both male $(n=6)$ and female $(n=5)$ replicates at day 7 using a 20x objective on a confocal microscope. Alpha-smooth muscle actin positive vessels had an increased diameter compared to capillaries. There was no difference between males and females in ACC diameter. There was also no difference between males and females or ligated and sham in capillary diameter. $\left(^{*}, \mathrm{p}<0.05\right.$ for alpha-smooth muscle actin positive vessels versus capillaries)

\section{Gelatin Vehicle for Cell Transplantation}

Because there was no difference in collateral capillary arterialization in males and females, we evaluated the ability of myogenic cell transplantation to augment arteriogenesis only in male mice. Myogenic cell transplantation is a potential solution to the need for improved PAOD treatments. Previous cell transplantation attempts in our laboratory utilized type I rat tail collagen as the vehicle.

Unfortunately, rat-tail collagen polymerized inconsistently- in some instances, the vehicles polymerized easily, while in others no polymerization ever occurred (unpublished observations). Therefore, to 
transplant myogenic cells in the spinotrapezius, we needed to develop a more consistent cell vehicle.

Gelatin was chosen as the vehicle material because it was easy to prepare, inexpensive, and biocompatible [74]. A gelatin solution was prepared, heated aseptically and then aliquoted into a 24-well plate for polymerization.

In addition to developing a protocol for sterile gelatin preparation, we also developed the vehicle composition. The first two iterations of the vehicle, $4 \%$ and $10 \%$ gelatin vehicles, depolymerized immediately when implanted beneath the spinotrapezius. Therefore, we added a crosslinking agent (1Ethyl-3-(3-dimethylaminopropyl) carbodiimide (EDC)) to gelatin preparations. To test the cross-linked gelatin acutely, both $4 \%$ and $10 \%$ gelatin vehicles cross-linked with either $6 \mathrm{mM}$ or $12 \mathrm{mM}$ EDC were incubated overnight at $37^{\circ} \mathrm{C}$ with $5 \% \mathrm{CO}_{2}$; all vehicles remained polymerized. Since both cross-linker concentrations remained polymerized, $6 \mathrm{mM}$ EDC was used in subsequent vehicle preparation to minimize the amount of cross-linker. With crosslinking maintaining gelatin polymerization, vehicles ( $4 \%$ and $10 \%$ gelatin cross-linked with $6 \mathrm{mM}$ EDC) were implanted deep to the spinotrapezius for 7 days $(\mathrm{n}=$ 2). After 7 days only the $10 \%$ gelatin vehicles remained polymerized, and there were no signs of infection or inflammation (Figure 8). Thus, we continued using the $10 \%$ gelatin cross-linked with $6 \mathrm{mM}$ EDC as the cell transplantation vehicle.
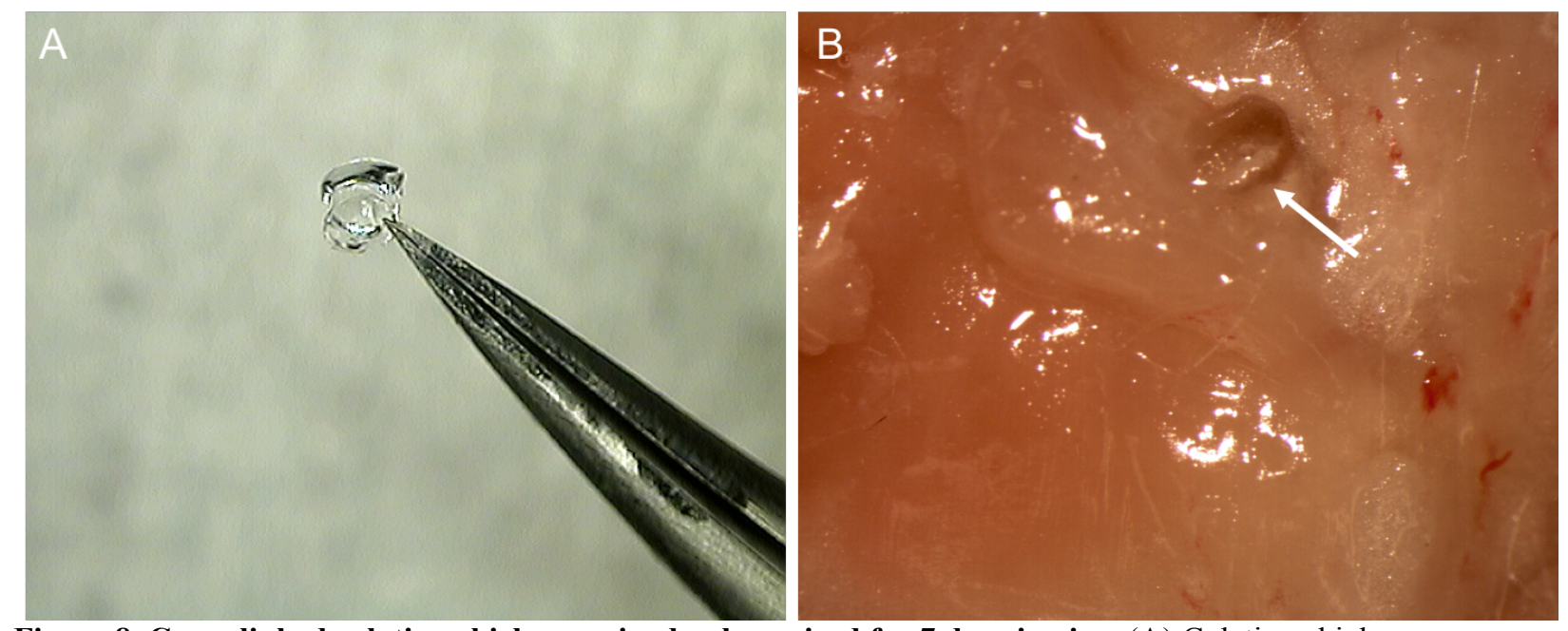

Figure 8. Cross-linked gelatin vehicle remained polymerized for 7 days in vivo. (A) Gelatin vehicles were punched with a $1.5 \mathrm{~mm}$ biopsy punch and implanted under the spinotrapezius. (B) Seven days later, the spinotrapezius was excised and the vehicle (white arrow) was still intact and there was no infection or inflammation. 
Finally, to assess the cytotoxicity of the cross-linked gelatin, we seeded them with fibroblasts and cultured for 12 hours, which is the same duration the myogenic cells would be cultured on the vehicle before transplantation. The 12-hour incubation time was chosen to allow the myogenic cells time to adhere to the gelatin while minimizing culture time in an attempt to possibly prevent the cells from differentiating any further because myogenic stem cells differentiate when they are cultured outside of their niche. After 12 hours, the fibroblasts had normal morphology and appeared adherent to the gelatin (Figure 9).

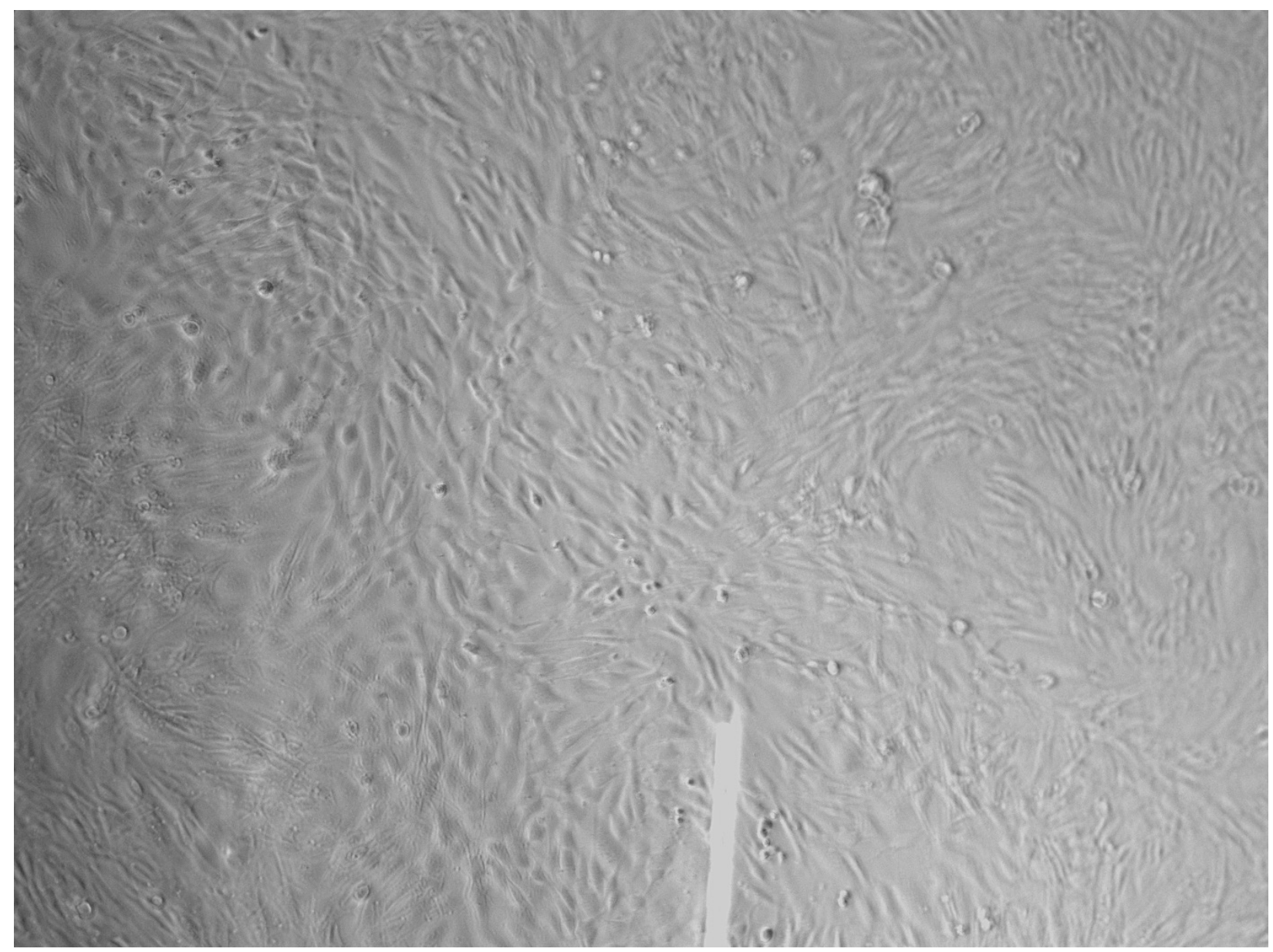

Figure 9. Fibroblasts have normal morphology after one day of culture on gelatin vehicle. Fibroblasts were cultured on the gelatin vehicle to evaluate cytotoxicity. After 24 hours, the cells had normal morphology and they appeared to have adhered to the vehicle. 


\section{Myogenic Cell Transplantation}

To test the hypothesis that myogenic cell transplantation increases collateral capillary arterialization we used confocal microscopy (Figure 10) to count and measure the diameter of collateral capillaries and ACCs between ischemic and perfused arteriolar trees.

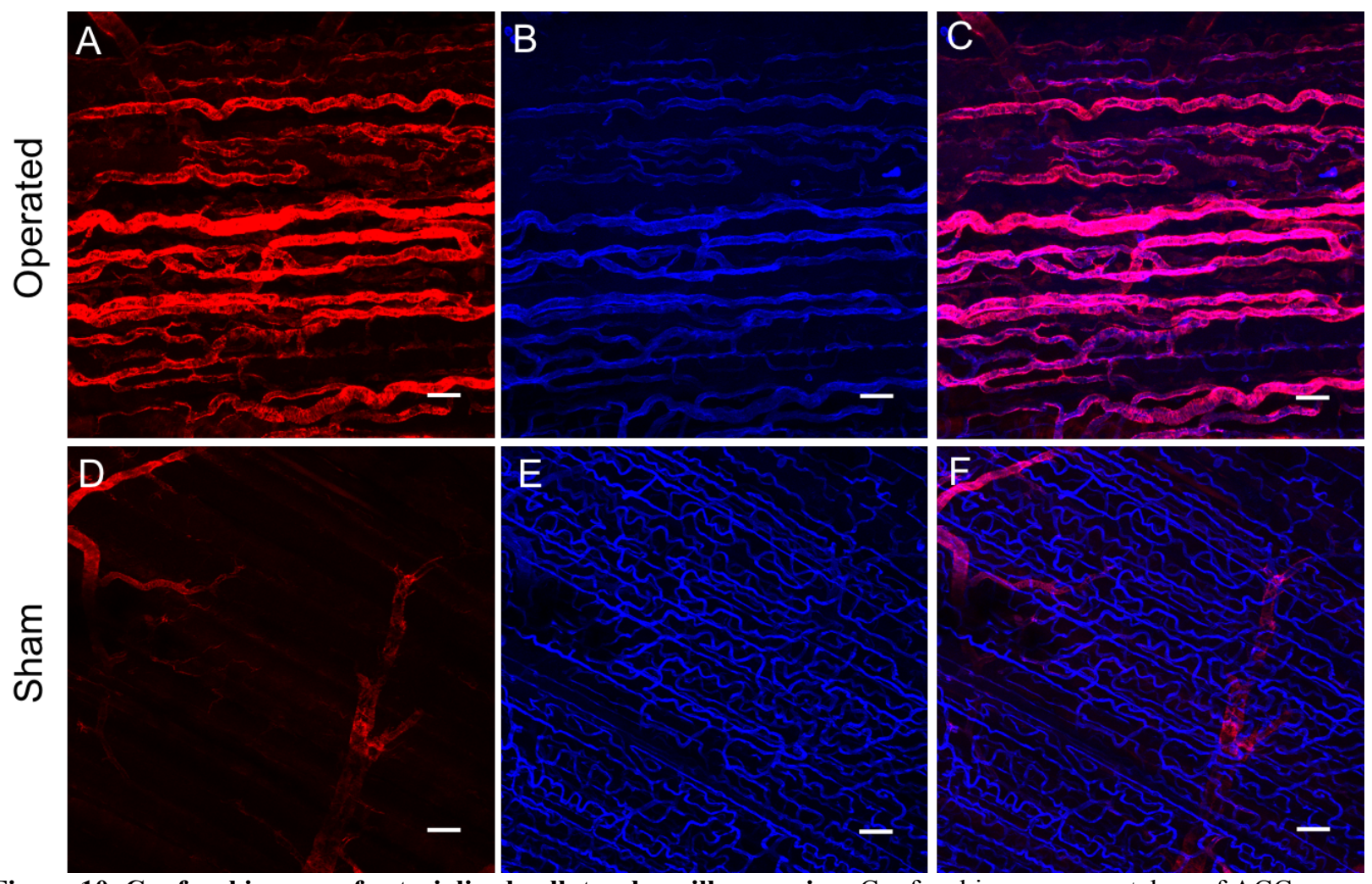

Figure 10. Confocal images of arterialized collateral capillary region. Confocal images were taken of ACCs between the ligated arteriolar tree and an adjacent arteriolar tree using a 20x objective. (A,D) Alpha-smooth muscle actin positive vessels show arterioles and ACCs. (B,E) Lectin positive vessels include both capillaries and ACCs. $(\mathrm{C}, \mathrm{F})$ Composite images combining $\mathrm{A}$ and $\mathrm{B}$ or D and E. A-C are images of an operated muscle and D-F are images of a sham muscle. Scale bars are $50 \mu \mathrm{m}$.

There was no difference in the total number of vessels between treatment groups $(12.4 \pm 0.6$

vessels with thrombin, $13.6 \pm 0.5$ vessels with myogenic cells, $12.5 \pm 0.3$ with vehicle, $13.7 \pm 0.6$ vessels with ligation only, and $13.6 \pm 0.3$ vessels in sham muscles) (Figure 11). To determine if myogenic cell transplantation increases ACC number, we counted alpha-smooth muscle actin positive vessels.

Thrombin, which enhances collateral capillary arteriogenesis [75], increased the ACC number $(9.3 \pm 0.5$ vessels versus $7.1 \pm 0.4$ vessels with myogenic cells, $6.6 \pm 0.1$ vessels with vehicle, and $7.5 \pm 0.3$ vessels 
with ligation only) (Figure 11). There was limited variation in ACC number between imaging regions, which suggests that 3 regions were sufficient for imaging.

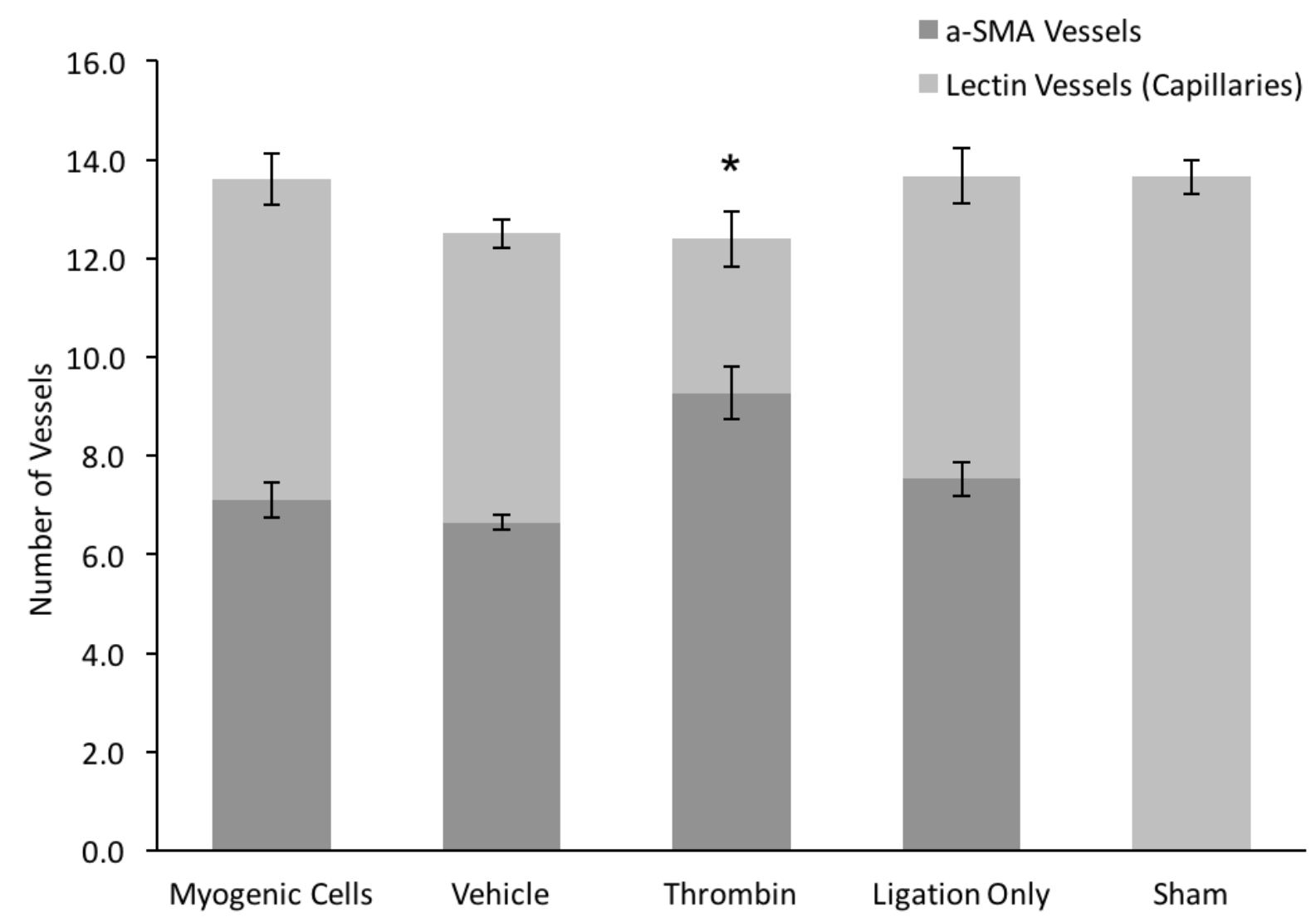

Figure 11. Thrombin increases number of arterialized collateral capillaries. Capillaries and ACCs were counted between ischemic and perfused trees in myogenic cells $(n=6)$, vehicle $(n=6)$, thrombin $(n=5)$, ligation only $(n=5)$, and sham $(n=24)$ replicates replicates at day 7 using a $20 x$ objective on a confocal microscope. Thrombin increased the number of ACCs. There was no difference in the total number of vessels between groups. $\left(^{*}, \mathrm{p}<0.05\right.$ for a-SMA positive vessels in thrombin treated versus all other groups)

Previously, thrombin decreased the number of ACCs in the region with the largest collateral [75]. To determine if myogenic cells have a similar effect, ACCs were counted in the region of the muscle with the largest collateral. While the previous ACC numbers were determined by counting ACCs in regions with average diameter ACCs (Figure 11), these measurements were determined by finding each replicate's largest ACC and counting the ACCs in that region. Both thrombin and myogenic cells decreased ACC number in this region $(8.5 \pm 1.2$ vessels and $9.7 \pm 1.2$ vessels versus $14.8 \pm 0.9$ vessels with vehicle and $13.7 \pm 0.9$ vessels with ligation only) (Figure 12). 

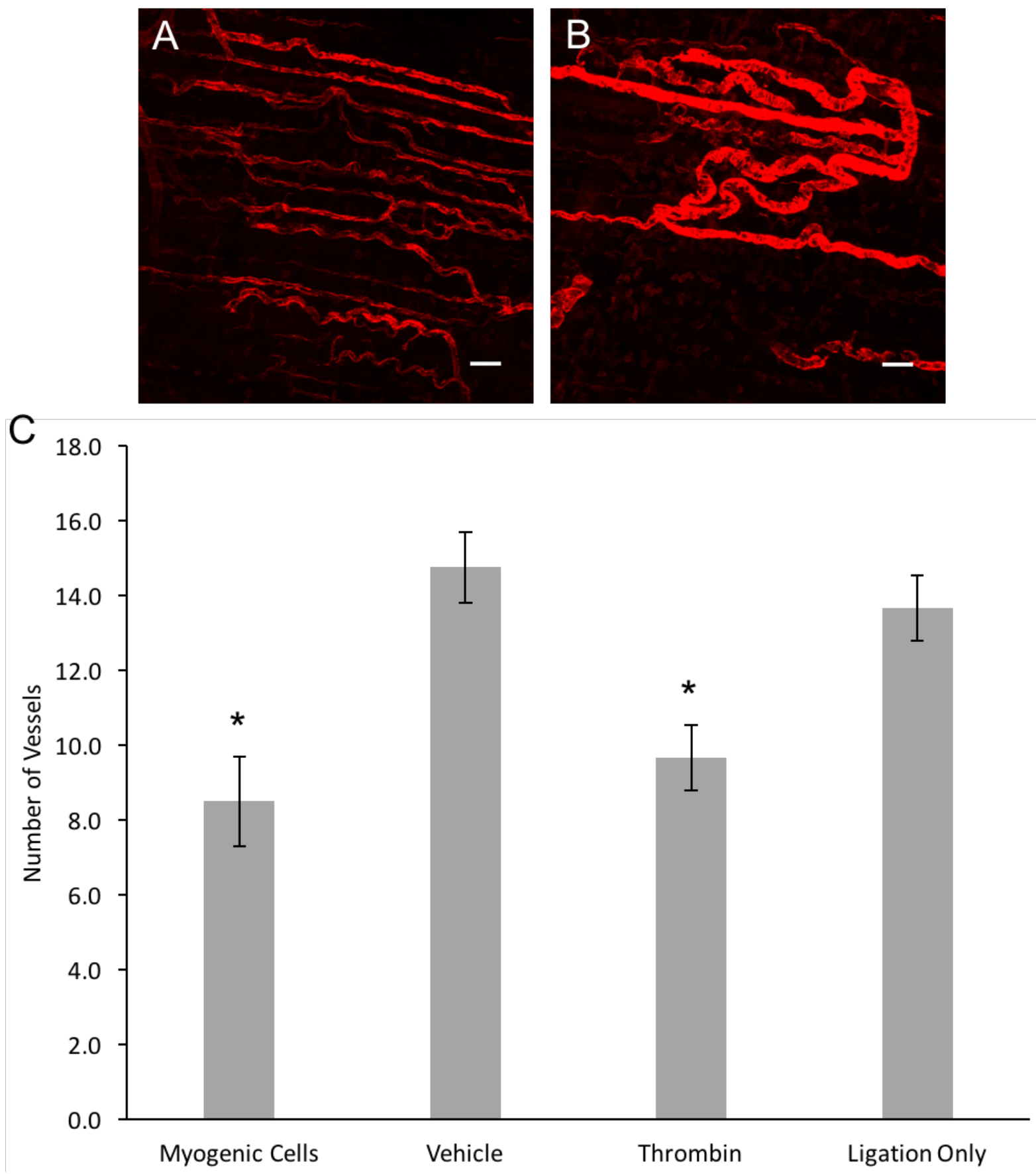

Figure 12. Thrombin decreases number of arterialized collateral capillaries in region with largest collateral. ACCs were counted between ischemic and perfused trees in mice without PECs in myogenic cells $(n=4)$, vehicle $(\mathrm{n}=3)$, thrombin $(\mathrm{n}=3)$, and ligation only $(\mathrm{n}=3)$ replicates replicates at day 7 using a $20 \mathrm{x}$ objective on a confocal microscope. (A) Example image of a region of average diameter ACCs (B) Example image of ACCs in the region with the largest collateral. (C) Myogenic cells and thrombin decreased the number of ACCs in the region with the largest collateral. $(*, p<0.05$ vs vehicle and ligation only) 
The decrease in vessel number suggests that myogenic cells could be affecting only selected collaterals, allowing them to enlarge slightly more than surrounding collaterals which increases the shear stress in that collateral and allows it to continue to enlarge faster than its neighbors. Therefore, to determine if myogenic cell transplantation increases overall ACC diameter, alpha-smooth muscle actin positive vessel diameters were measured. Myogenic cells increased ACC diameter $(12.1 \pm 0.8 \mu \mathrm{m}$ and $11.9 \pm 0.5 \mu \mathrm{m})$ compared to the vehicle $(10.1 \pm 0.4 \mu \mathrm{m})$ and ligation only $(10.5 \pm 0.4 \mu \mathrm{m})$, but not compared to thrombin $(11.9 \pm 0.5 \mu \mathrm{m})$ (Figure 10). There was no difference in capillary size between the ligated and sham muscles or between treatment groups (Figure 13). There was also limited variation in ACC diameter between imaging regions, which suggests that 3 regions were sufficient for imaging.

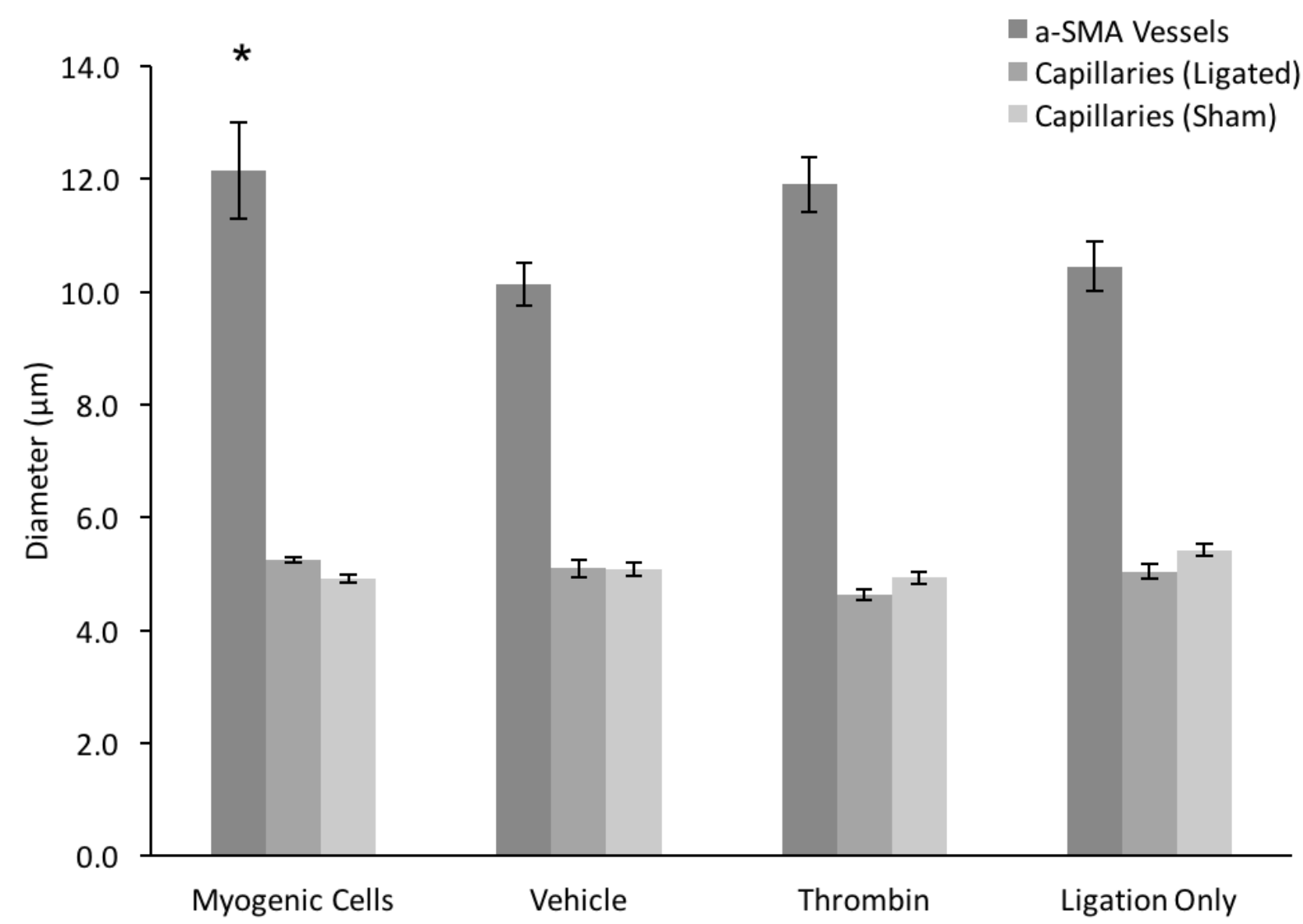

Figure 13. Myogenic cells increase arterialized collateral capillary size. Capillary and ACC diameters were measured between two arterial trees in myogenic cells $(n=6)$, vehicle $(n=6)$, thrombin $(n=5)$, and ligation only $(n=5)$ replicates replicates at day 7 using a 20x objective on a confocal microscope. Myogenic cells increased ACC diameter compared to vehicle and ligation only. There was no difference in capillary size between ligated and sham muscles or between treatment groups. $(*, p<0.05$ versus vehicle and ligation only) 
Even though thrombin did not increase the average ACC diameter, previous work demonstrated that thrombin increases the maximum ACC diameter [75], so we determined if myogenic cells have a similar effect. Both myogenic cells and thrombin increased the maximum ACC diameter $(29.9 \pm 3.4 \mu \mathrm{m}$ with myogenic cells and $27.9 \pm 2.0 \mu \mathrm{m}$ with thrombin versus $17.9 \pm 1.7 \mu \mathrm{m}$ with vehicle and $18.9 \pm 1.1$ $\mu \mathrm{m}$ with ligation only) (Figure 14).

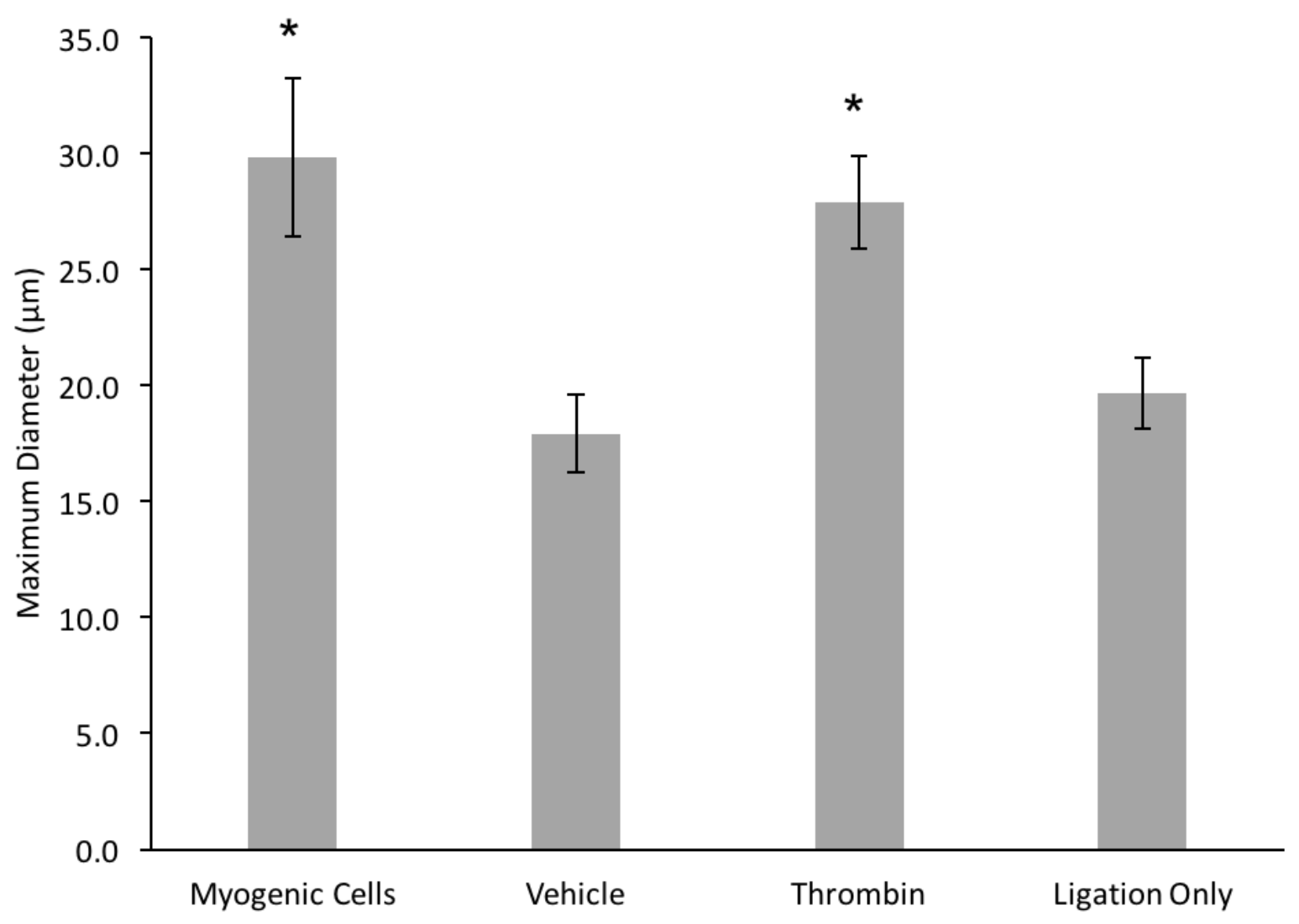

Figure 14. Myogenic cells increase maximum arterialized collateral capillary diameter. The largest ACC diameter was measured in myogenic cells $(n=4)$, vehicle $(n=4)$, thrombin $(n=3)$, and ligation only $(n=3)$ replicates at day 7 using a 20x objective on a confocal microscope. Myogenic cells and thrombin replicates increased the maximum diameter ACC compared to the other two groups. ( ${ }^{*}, \mathrm{p}<0.05$ vs gelatin and ligation only)

Some Balb/C mice have pre-existing collaterals (PECs), which may affect arteriogenesis because blood flows along the path of least resistance [76], and arteriogenesis occurs in response to increased shear stress due to increased blood flow. Therefore, it is possible that a PEC 'steals' flow and the majority of the remodeling happens in the PEC. To determine if PECs influenced the number of ACCs, mice were analyzed in two groups: mice with PECs ( $\mathrm{n}=2$ for all groups) and mice without PECs ( $\mathrm{n}=4$ for 
myogenic cells and vehicle, $\mathrm{n}=3$ for thrombin and ligation only). In mice without PECs, thrombin increased the number of ACCs $(9.9 \pm 0.5$ vessels versus $7.1 \pm 0.5$ vessels with myogenic cells, $6.5 \pm 0.2$ vessels with vehicle, and $7.7 \pm 0.1$ vessels with ligation only) (Figure 15). There was no difference in the number ACCs between mice without PECs and mice with PECs for all groups (Figure 15).

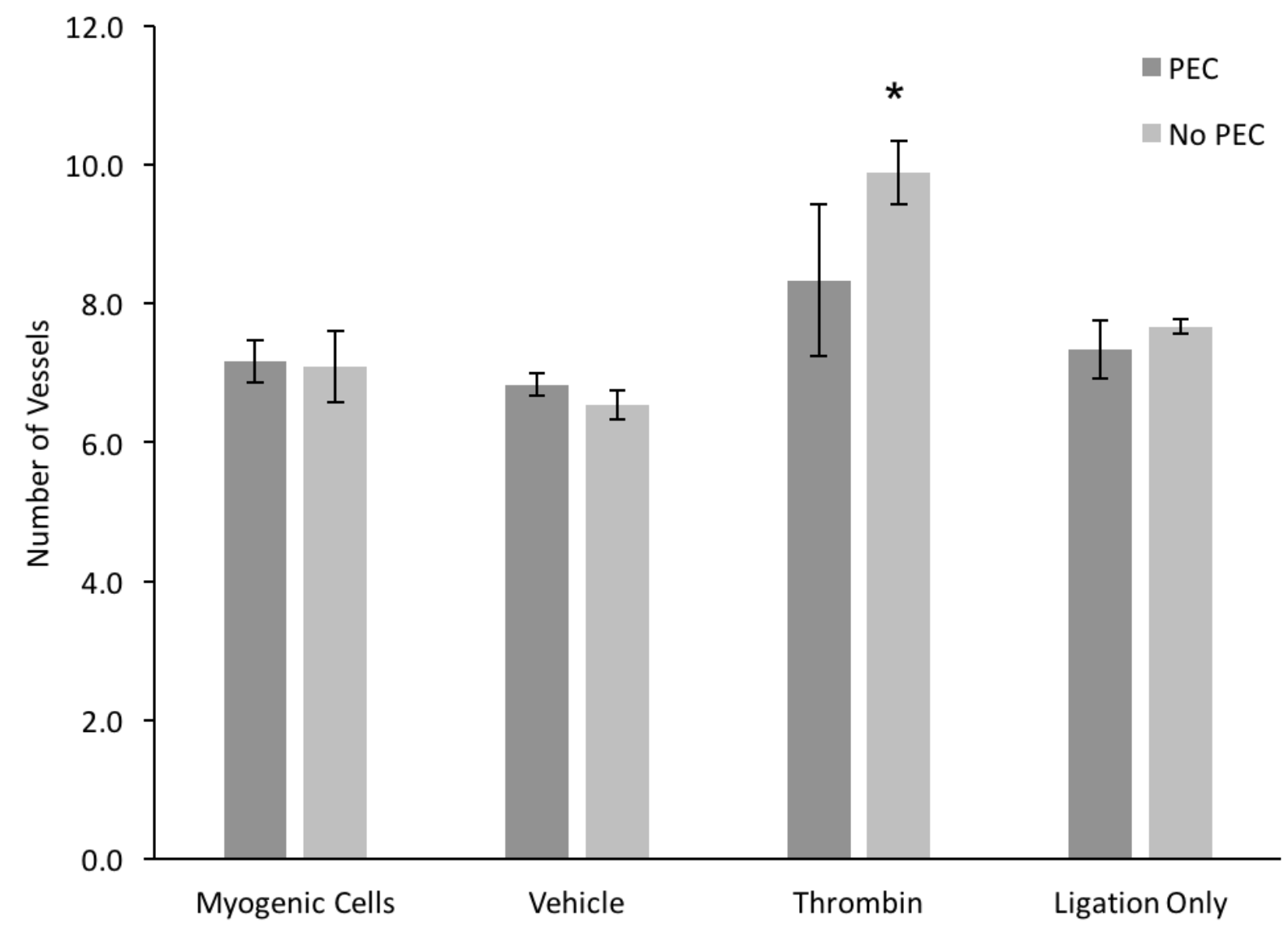

Figure 15. Thrombin increases number of arterialized collateral capillaries in mice without PECs. ACCs were counted between ischemic and perfused trees in myogenic cells (PEC: $n=2$, No PEC: $n=4$ ), vehicle (PEC: $n=2$, No PEC: $n=4$ ), thrombin (PEC: $n=2$, No PEC: $n=3$ ), and ligation only (PEC: $n=2$, No PEC: $n=3$ ) replicates at day 7 using a 20x objective on a confocal microscope. There was no difference in the number of ACCs in mice with a PEC and mice without a PEC. In mice without a PEC, thrombin increased the number of ACCs. $\left(^{*}, p<0.05\right.$ versus all other groups)

To determine if PECs influence ACC size, ACC diameters were compared in mice with PECs and in mice without PECs. Mice without PECs trended towards a larger ACC diameter than mice without PECs (Figure 16). Additionally, in mice without a PEC, myogenic cells increased ACC diameter compared to other treatment groups $(13.7 \pm 1.2 \mu \mathrm{m}$ with myogenic cells, $10.5 \pm 0.5 \mu \mathrm{m}$ with vehicle, 13.4 
$\pm 0.6 \mu \mathrm{m}$ with thrombin, and $12.0 \pm 0.5 \mu \mathrm{m}$ with ligation only), and thrombin increased diameter compared to vehicle (Figure 16).

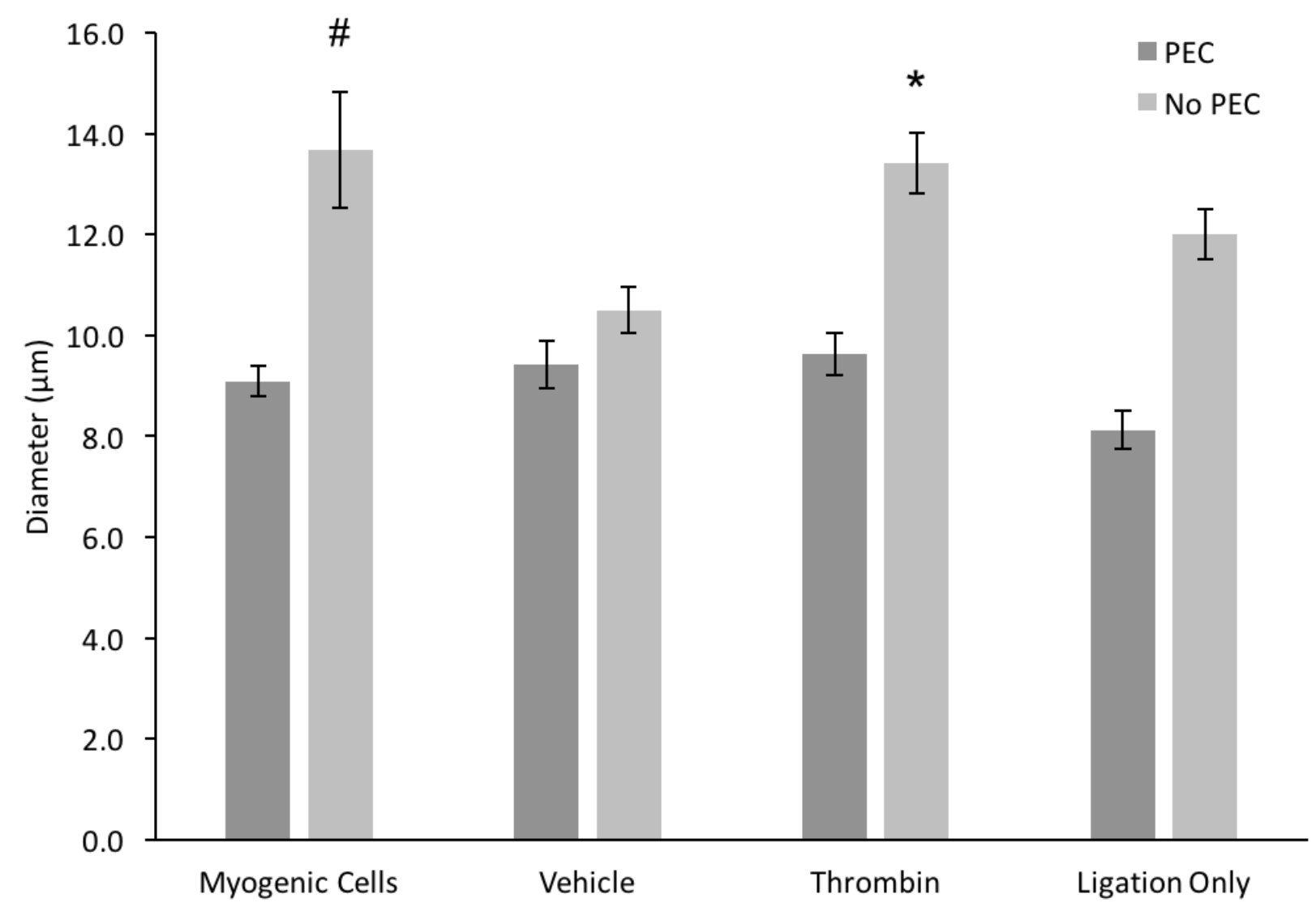

Figure 16. Myogenic cells increase arterialized collateral capillary diameter in mice without PECs. Capillary and ACC diameters were measured between two arterial trees in myogenic cells (PEC: $n=2$, No PEC: $n=4$ ), vehicle (PEC: $n=2$, No PEC: $n=4$ ), thrombin (PEC: $n=2$, No PEC: $n=3$ ), and ligation only (PEC: $n=2$, No PEC: $n=3$ ) replicates at day 7 using a 20x objective on a confocal microscope. There was a trend toward an increased ACC size in mice without a PEC. Myogenic cell transplantation increased ACC diameter. (\#, $\mathrm{p}<0.05$ versus vehicle, thrombin, and ligation only; *, $\mathrm{p}<0.05$ versus vehicle)

Because myogenic cells increase arteriogenesis and macrophages are an important contributor to arteriogenesis $[77,78,79]$, we tested the hypothesis that myogenic cells increase ACC diameter by increasing macrophage recruitment. We used confocal microscopy to count the number of CD68 positive cells in the region in which ACCs form anastomoses between the ischemic and perfused arteriolar trees as well as to count the number of vessel associated CD68 cells (Figure 17). 


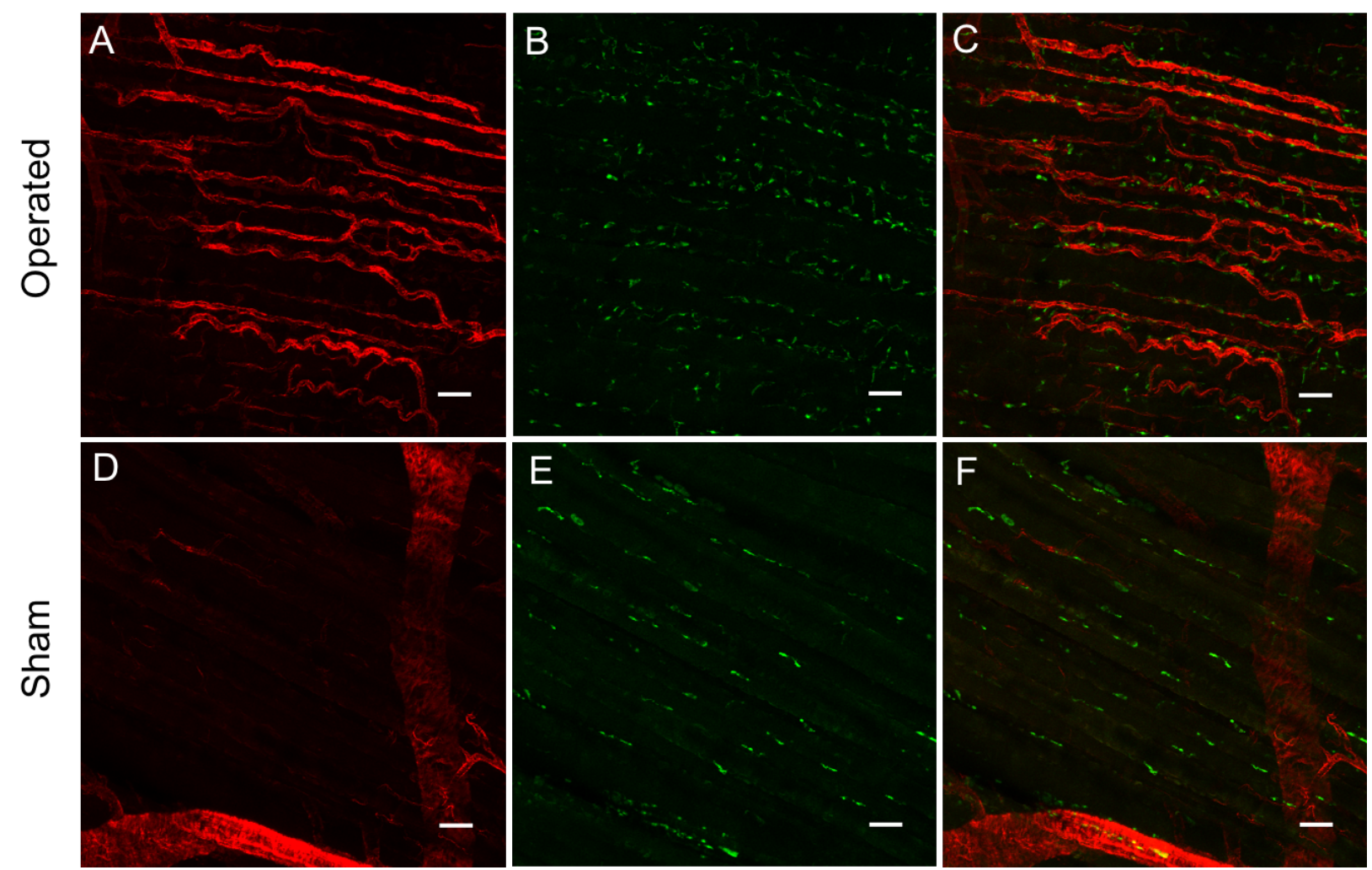

Figure 17. Confocal images of CD68 positive cells. Confocal images were taken of CD68 positive cells between the ligated arteriolar tree and an adjacent arteriolar tree using a 20x objective. (A,D) Alpha-smooth muscle actin positive vessels show arterioles and ACCs. (B,E) CD68 positive cells indicate macrophages. (C,F) Composite images combining $\mathrm{A}$ and $\mathrm{B}$ or $\mathrm{D}$ and $\mathrm{E}$. A-C are images of an operated muscle and D-F are images of a sham muscle. Scale bar is $50 \mu \mathrm{m}$.

Ligation increased CD68 positive cells (Figure 18), but myogenic cells and thrombin did not enhance this effect $(16.0 \pm 0.5$ cells with myogenic cells, $15.8 \pm 0.4$ cells with vehicle, $15.3 \pm 0.5$ with thrombin, and $15.5 \pm 0.5$ with ligation only) (Figure 18). Myogenic cells also did not affect the number of vessel associated cells $(16.9 \pm 0.8$ cells with myogenic cells, $14.6 \pm 0.7$ cells with vehicle, $16.1 \pm 0.7$ with thrombin, and $17.1 \pm 0.8$ with ligation only) (Figure 18). There was limited variation in CD68 content between imaging regions, which suggests that 3 regions were sufficient for imaging. 


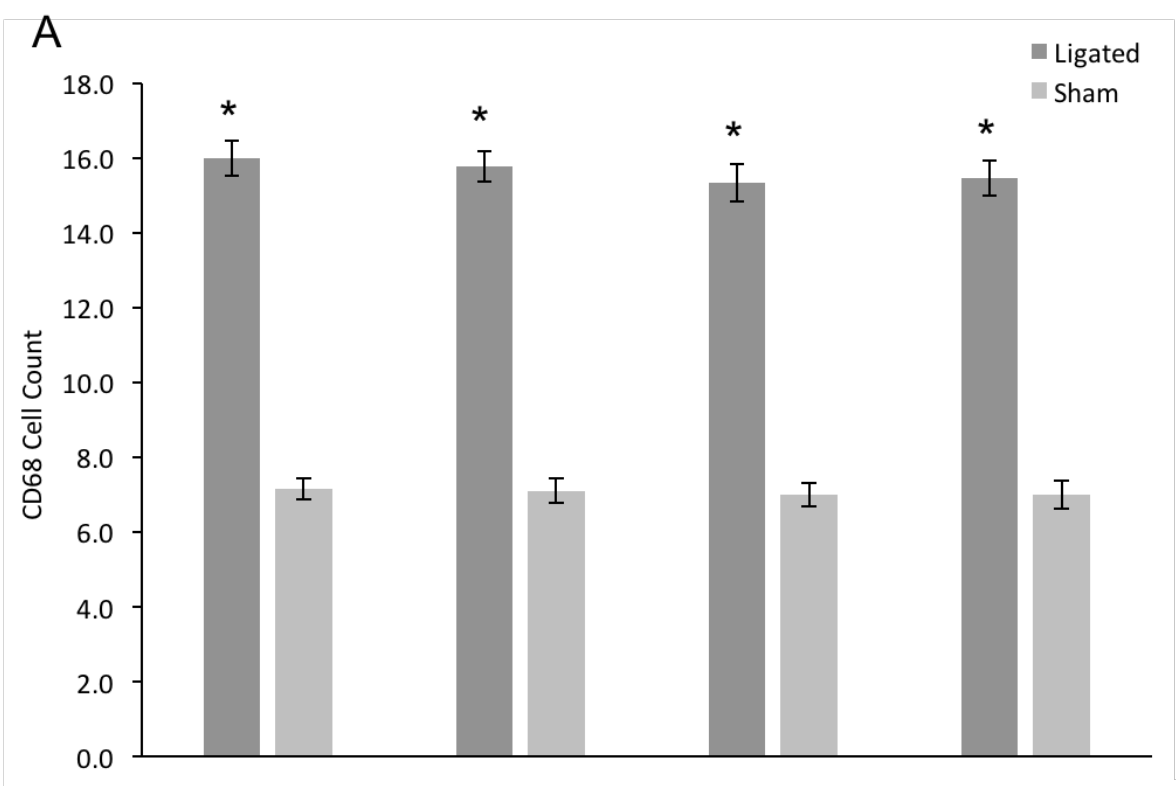

B

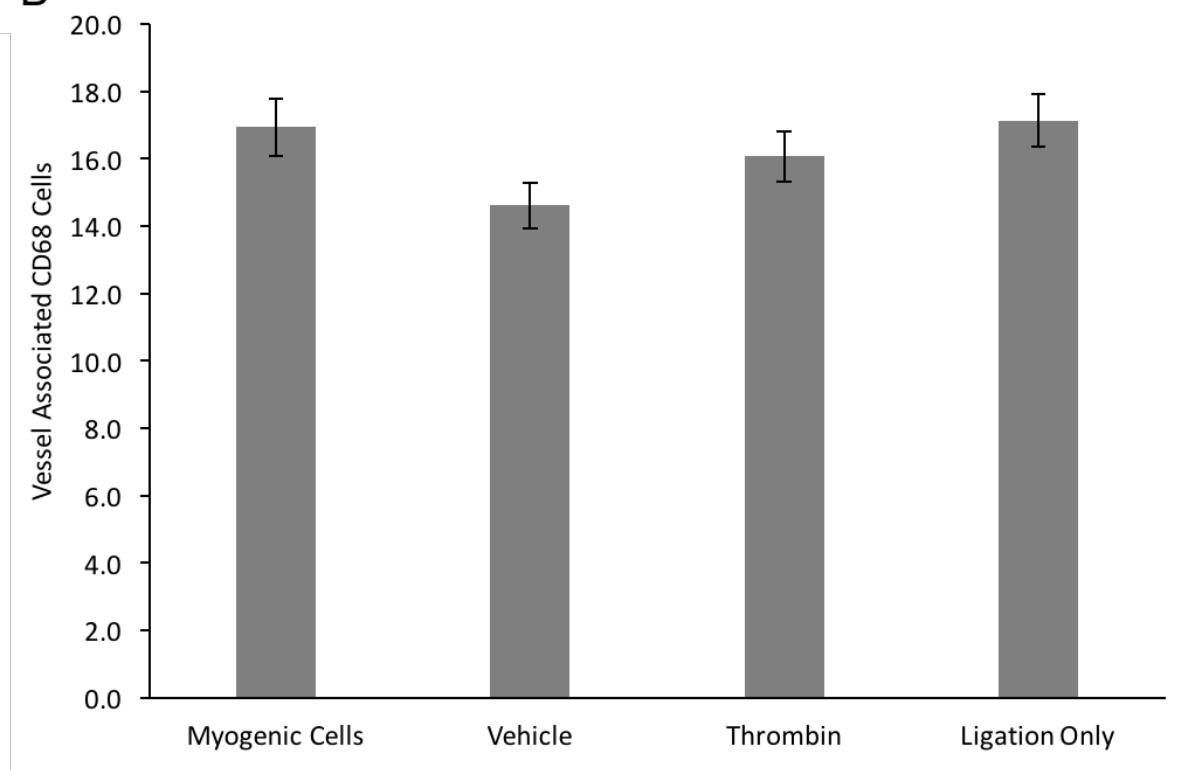

Figure 18. Myogenic cell transplantation has no effect on CD68 cell count. CD68 positive cells were counted between two arterial trees in myogenic cells $(n=6)$, vehicle $(n=6)$, thrombin $(n=5)$, and ligation only $(n=5)$ replicates replicates at day 7 using a 20x objective on a confocal microscope. There was no difference in the number of CD68 cells between groups. $(*, p<0.05$ vs sham) 


\section{DISCUSSION}

Pre-existing collaterals improve the prognosis in patients with ischemic diseases, but some patients never develop angiographically-identifiable collateral vessels [3,4,5,8,9]. Additionally, females appear to have a worse prognosis than males for peripheral arterial occlusive disease (PAOD) $[64,65,66]$, possibly due to impaired collateral development [70]. Collateral vessels develop through arteriogenesis in response to increased shear stress that triggers a cascade of events involving endothelial cell activation, macrophage recruitment, matrix degradation, and smooth muscle/endothelial cell proliferation.

Arteriogenesis results in a collateral arteriole with an expanded diameter that helps restore blood flow to the ischemic area. Because current treatments for severe PAOD have limited positive outcomes [2], alternative treatments to promote arteriogenesis are needed. Cell transplantation is a promising treatment for PAOD, but there has been limited clinical success for multiple cell types including bone marrow mononuclear cells, bone marrow mesenchymal cells, and endothelial progenitor cells $[29,30,39,40,44,45]$. Tissue resident stem cells, such as myogenic stem cells, have not been investigated as a treatment for PAOD, but they have potential to promote arteriogenesis $[46,51,55,56]$. Therefore, we determined if arterialized collateral capillary (ACC) formation was impaired in female mice compared to male mice, developed a myogenic cell transplantation protocol, and tested the hypotheses that myogenic cell transplantation enhanced arteriogenesis and macrophage recruitment.

Seven days post ligation, ACCs on the operated muscle were significantly larger in diameter than collateral capillaries in the sham muscle, demonstrating that outward remodeling occurred. There was also no difference in total number of vessels in the ligated and sham muscles, demonstrating that the ACCs developed through capillaries outwardly remodeling and recruiting smooth muscle cells, as observed previously $[15,80]$. Interestingly, there was no difference between males and females in ACC diameter or number. The lack of difference between male and female mice could have occurred because the mice used in this study were healthy, and clinical sex differences are observed in patients who are often elderly with comorbidities and other risk factors $[65,66,67]$. Unfortunately, we do not know of any arteriogenesis investigations in female mice with ischemic disease comorbidities, but arteriogenesis is 
impaired in both aged and diabetic mice compared to healthy models [81,82], and female ApoE ${ }^{-/-}$mice exhibit endothelial dysfunction compared to male mice $[83,84,85]$ and have larger atherosclerotic legions $[86,87]$. To determine if comorbidities affect arteriogenesis in female mice, a diabetic or aged mouse model could be investigated.

Seven days post ligation, thrombin increased the number of ACCs compared to all other treatment groups, including myogenic cells. However, the increase in ACC number with thrombin treatment is not necessarily an advantage because it may not result in a more effective restoration of blood flow. In the mouse hindlimb ischemia model, the density of blood vessels in the hindlimb peaks seven days post ligation, and as the number of vessels decreased, the hindlimb perfusion stayed the same [88], suggesting that the increased number of vessels is not necessary to restore bloodflow. With other arteriogenic factors, such as Fibroblast Growth Factor (FGF) and Vascular Endothelial Growth Factor (VEGF), the initial response to treatment is accelerated $[89,90,91]$, but at later timepoints, there was no difference in perfusion [92], and the excess vessels formed during the initial accelerated development appear to be leaky and therefore not functional [92] . Previous work has demonstrated that thrombin augments the remodeling process at early timepoints similar to FGF and VEGF $[75,93]$. The administration of thrombin may promote the growth of blood vessels that are unnecessary to restore shear stress in the collateral zone, and therefore will regress at a later timepoint. Although myogenic cells did not increase in the number of ACCs, increasing the number of ACCs may not be necessary for ischemic recovery. To determine if thrombin's effect is temporary, a study with longer endpoints such as 14 or 28 days could be performed.

Even though myogenic cells had no effect on the number of ACCs, both myogenic cells and thrombin increased ACC diameter. The maximum ACC diameter was also larger with both thrombin and myogenic cells. Additionally, in the region of the muscle with the largest collateral, both treatments decreased the number of ACCs compared to the other two treatment groups. Previous work has demonstrated that thrombin augments the remodeling of only certain collaterals [75]. The results from this study suggest that myogenic cell transplantation has a similar effect to thrombin injections, which 
supports our hypothesis that myogenic cells would augment arteriogenesis. Myogenic cells secrete factors such as matrix metalloproteinases (MMPs) and VEGF $[94,95]$ which are involved in arteriogenesis signaling and could be secreting these factors to accelerate arteriogenesis. VEGF is responsible for endothelial cell proliferation during arteriogenesis $[96,97,98]$, and MMPs are responsible for degrading the matrix during vessel remodeling [14]. In a future study, to determine if myogenic cells are increasing arteriogenesis by secreting arteriogenic factors, we could analyze conditioned media to determine what proteins the myoblasts are secreting.

Myogenic cells are not the only factor that can affect arteriogenesis in certain collaterals, preexisting collaterals (PECs), which are present in some mice from the Balb/C strain, could also affect which collaterals experience arteriogenesis. To determine if PECs affect collateral capillary arterialization, mice with PECs were analyzed separately from mice without PECs. There was a trend towards smaller ACCs in mice with PECs. Blood flows along the path of least resistance [76], so after a ligation blood flow would be redirected mainly through the PEC since it is the lowest resistance vessel. Because the PEC has the largest increase in blood flow and therefore the largest increase in shear stress, the PEC would be the main arteriole to undergo remodeling. While mice with a PEC appeared to have a trend towards reduced collateral capillary arteriogenesis, they did experience some collateral capillary arteriogenesis. Because the PEC was smaller than the original feed artery, it is possible that it could not normalize shear stress on its own, so some of the capillaries could have experienced an increase in shear stress causing some collateral capillary arteriogenesis [15]. In mice with a PEC, the PEC could have been the main collateral and therefore could have experienced the majority of the remodeling. While PECs may have an effect on ACC formation, including mice with a PEC in the initial vessel count and vessel diameter analysis may not have affected the results because the same conclusions about the effects of myogenic cells were drawn when mice with a PEC were excluded. To determine if the PEC experiences the largest increase in blood flow following ligation, we could utilize an imaging technique that allows for blood flow quantification such as laser speckle flowmetry [99]. In future studies, more replicates would 
be included to determine if there are any statistical differences between mice with PECs and mice without PECs.

In addition to secreting arteriogenic factors, myoblasts also recruit white blood cells $[52,53]$. To determine if myogenic cell transplantation increased macrophage recruitment, the number of CD68 positive cells were counted. As expected, ligation increased the number of macrophages in the region with ACCs. Following ligation, the increased shear stress in the collateral vessel activates the endothelium, causing it to secrete MCP-1 which activates monocytes, enhancing their adhesion to the endothelium [100]. Once activated, monocytes extravasate into the tissue and become macrophages [100]. Without macrophage recruitment, there is minimal arteriogenesis $[77,78,79]$, so it was expected that the ligated muscle would see an increase in macrophages. However, both myogenic cells and thrombin had no effect on macrophage recruitment. The lack of difference between the groups could have occurred because the seven day timepoint is too long after ligation to see macrophage number differences. Macrophages are recruited to the collateral site early in arteriogenesis, and the number of macrophages peaks three days after ligation and is significantly decreased by day $7[100,101]$. Additionally, in thrombin treated mice, at three days post ligation there is only a trend towards an increase in the number of macrophages when compared to a control injection [75]. This suggests that arteriogenic agents such as thrombin or myogenic cells may be able to increase macrophage recruitment. Therefore, there may have been a difference in the number of macrophages between groups at 3 days post ligation that was gone by the 7 day timepoint used in this study. In a future study, to determine if myogenic cells increase macrophage recruitment earlier than 7 days post ligation, a 3 day timepoint could be added.

Another possible reason for the failure of myogenic cells to affect macrophage number is that there may be differences in the numbers of each type of macrophage. Macrophages exist on a spectrum that ranges from classically activated to alternatively activated [102]. Classically activated macrophages are also referred to as M1 macrophages and are pro-inflammatory, while alternatively activated macrophages are also referred to as M2 macrophages and are involved in tissue remodeling [102]. M2 macrophages are the main contributor to arteriogenesis and the main type of macrophage at the collateral 
site $[103,104]$. Because a general macrophage marker, CD68, was used in this study, the number of each type of macrophage could not be determined. It is possible that myogenic cells increased the number of M2 macrophages, but this increase could not be observed. To determine if there are more M2 macrophages a stain like CD206 that is specific to M2 macrophages [73] could be used in addition to the CD68 stain.

There were a few limitations to this study. The sample size was small both for comparing mice with PECs to mice without PECs, and for comparisons made with only mice without PECs. When comparing mice with PECs to mice without PECs, only general trends could be observed. In the future, to draw statistical conclusions about the effect of a PEC on ACCs, a sample size of 6 mice with a PEC would be needed to have a power of 0.8 . In addition, 6 replicates would also be needed to have power of 0.8 for comparisons between treatment groups that were made only in mice without PECs, such as the maximum collateral diameter and the number of ACCs in the region with the largest collateral.

Since this study was the first cell transplantation study in the spinotrapezius performed in this lab, the protocol could still be optimized. It is possible that myogenic cells did not increase the number of ACCs like thrombin because the number of cells transplanted was not optimized. In this study, $9.3 \times 10^{3}$ cells was chosen based on results from previous work done in the lab in the mouse hindlimb, which used more cells but had disorganized blood vessel growth $[105,106]$, as well as the number of cells available at the time of surgery. This study was the first cell transplantation study performed in the spinotrapezius, so a cell dose response study may be necessary to determine the optimal number of cells and to determine if changing the number of cells affects the number of ACCs. To determine the optimal number of cells to transplant, we could perform a dose response study with 1,000 to 1 million cells based on previous cell transplantation studies $[88,107,108]$.

The vehicle could also be optimized for future cell transplantation studies. Gelatin was chosen for this initial cell transplantation study because it is biocompatible, easy to prepare, and inexpensive [74]. However, there are some disadvantages to gelatin. Ideally, the gelatin vehicle would have a modulus close to the modulus of muscle so that the myogenic cells are in an environment similar to their native ECM 
[109], but because gelatin must be boiled, it is difficult to create gelatin vehicles with a consistent gelatin concentration and therefore a consistent modulus. We chose the concentration of gelatin and EDC for the vehicles based on literature that measured the gelatin modulus [110,111,112,113,114], but were unable to determine if the modulus of the final vehicle was close to that of muscle. Another disadvantage to the gelatin vehicles was the use of a biopsy punch to create appropriately sized vehicles. Thick gelatin vehicles were used because they worked with the biopsy punch and thinner gels did not, but thinner gels may have been more beneficial. Also, because the biopsy punch was used during surgery when the cells were already on the gelatin vehicles, it was difficult to obtain an accurate cell count. In future studies, extra punches could be prepared and stained with BBI or a myogenic stem cell marker to estimate the number of transplanted myogenic stem cells. Additionally, during the follow-up procedure, the punches could be explanted and stained with the same stains to determine how many myogenic stem cells remained in the vehicle. It is also possible that the biopsy punch and the use of the forceps to place the gelatin vehicles beneath the spinotrapezius damaged some of the cells, despite performing both steps gently. To determine if implanting the vehicle damaged the cells, we could simulate the vehicle implantation and then use a live/dead stain on the vehicle. However, a construct that requires less interaction with surgical instruments would be ideal. A solution to the problems encountered with gelatin is the use of a thermoreversible hydrogel, which can be engineered with a specific modulus $[115,116,117]$, and the cells can be added to the exact injected volume of vehicle. Since the vehicle would be injected directly into the mouse, it can be designed such that the syringe minimally damages the cells during construct delivery [118]. Additionally, with the thermoreversible gel the cells would be dispersed throughout the gel rather than on the surface as they are with the gelatin. Since the cells would be throughout the gel, they may be more protected from damage during transplantation than they are with gelatin, but any secreted factors would have to diffuse through the gel to reach the muscle.

Myogenic cells increased ACC diameter and maximum ACC diameter, and decreased the number of ACCs in the region surrounding the maximum diameter ACC. This supports the hypothesis that myogenic cell transplantation is a potential arteriogenesis-enhancing treatment for PAOD. However, 
myogenic cells did not affect macrophage recruitment. The methods used in this study still need optimization to determine if myogenic cells affect macrophage recruitment at earlier time points or in specific populations. Additionally, we found no differences in arteriogenesis between males and females. In future studies, diseased animal models can be utilized to determine if impaired arteriogenesis is involved in the clinical sex differences seen with PAOD. 


\section{REFERENCES}

1. Allison, M. A. et al. Ethnic-Specific Prevalence of Peripheral Arterial Disease in the United States. Am J Prev Med 32, 328-333 (2007).

2. Norgren, L. et al. Inter-Society Consensus for the Management of Peripheral Arterial Disease ( TASC II ). J. Vasc. Surg. 45, S5A-S67A (2007).

3. Perez-Castellano, N. et al. Influence of Collateral Circulation on In-Hospital Death From Anterior Acute Myocardial Infarction. J. Am. Coll. Cardiol. 31, 512-518 (1998).

4. Sabia, P. et al. An Association Between Collateral Blood Flow and Myocardial Viability in Patients with Recent Myocardial Infarction. N. Engl. J. Med. 327, 1825-1831 (1992).

5. Nathoe, H. M. et al. Determinants and Prognostic Significance of Collaterals in Patients Undergoing Coronary Revascularization. Am. J. Cardiol. 98, 31-35 (2006).

6. Mcdermott, M. M. et al. Collateral vessel number, plaque burden, and functional decline in peripheral artery disease. (2014). doi:10.1177/1358863X14540362

7. Mcdermott, M. M. et al. Proximal Superficial Femoral Artery Occlusion, Collateral Vessels, and Walking Performance in Peripheral Artery Disease. 6, (2013).

8. Tatli, E., Altun, A. \& et al. Coronary collateral vessel development after acute myocardial infarction. Exp. Clin. Cardiol. 12, 97-99 (2007).

9. Fujita, M., Sasayama, S. \& et al. Coronary Collateral Development After Acute Myocardial Infarction. Clin. Cardiol. 11, 525-528 (1988).

10. Peripheral artery disease (PAD): Diagnosis and Treatment. Mayo Clinic (2017).

11. Treatment Options for Peripheral Artery Disease. Medtronic (2016).

12. Scholz, D. et al. Contribution of Arteriogenesis and Angiogenesis to Postocclusive Hindlimb Perfusion in Mice. J Mol Cell Cardiol 34, 775-87 (2002).

13. Helisch, A. \& Schaper, W. Arteriogenesis The Development and Growth of Collateral Arteries. Microcirculation 10, 83-97 (2003).

14. Carmeliet, P. Mechanisms of angiogenesis and arteriogenesis. Nat. Med. 6, 389-395 (2000).

15. Gabhann, F. Mac \& Peirce, S. M. Collateral capillary arterialization following arteriolar ligation in murine skeletal muscle. Microcirculation 17, 333-47 (2010).

16. Heil, M., Eitenmuller, I., Schmitz-Rixen, T. \& Schaper, W. Arteriogenesis versus angiogenesis: similarities and differences. J Cell Mol Med 10, 45-55 (2006).

17. Lazarous, D. F. et al. Basic Fibroblast Growth Factor in Patients With Intermittent Claudication: Results of a Phase I Trial. J. Am. Coll. Cardiol. 36, 1239-1244 (2000).

18. Rajagopalan, S. et al. Use of a Constitutively Active Hypoxia-Inducible Factor-1 $\smile$ Transgene as a Therapeutic Strategy in No-Option Critical Limb Ischemia Patients: Phase I Dose-Escalation 
Experience. Circulation 115, 1234-1243 (2007).

19. Baumgartner, I. et al. Constitutive Expression of phVEGF 165 After Intramuscular Gene Transfer Promotes Collateral Vessel Development in Patients With Critical Limb Ischemia. Circulation 97, 1114-1123 (1998).

20. Powell, R. J. et al. Safety and efficacy of patient specific intramuscular injection of HGF plasmid gene therapy on limb perfusion and wound healing in patients with ischemic lower extremity ulceration : Results of the HGF-025 trial. J. Vasc. Surg. 52, 1525-1530 (2010).

21. Gu, Y., Ding, D., Kim, J. \& Hahn, W. A phase I clinical study of naked DNA expressing two isoforms of hepatocyte growth factor to treat patients with critical limb ischemia. J. Gene Med. 13, 602-610 (2011).

22. Kusumanto, Y. H., Weel, V. V. A. N., Mulder, N. H., Smit, A. J. \& Dungen, J. J. A. M. V. A. N. D. E. N. Treatment with Intramuscular Vascular Endothelial Growth Factor Gene Compared with Placebo for Patients with Diabetes Mellitus and Critical Limb Ischemia: A Double-Blind Randomized Trial. Hum. Gene Ther. 17, 683-691 (2006).

23. Rajagopalan, S. et al. Regional Angiogenesis With Vascular Endothelial Growth Factor in Peripheral Arterial Disease. Circulation 108, 1933-1939 (2003).

24. Nikol, S. et al. Therapeutic Angiogenesis With Intramuscular NV1FGF Improves Amputation-free Survival in Patients With Critical Limb Ischemia. Mol. Ther. 16, 972-978 (2008).

25. Creager, M. A. et al. Effect of Hypoxia-Inducible Factor-1 $\backsim$ Gene Therapy Intermittent Claudication. Circulation2011 124, 1765-73 (2011).

26. Belch, J. et al. Effect of fibroblast growth factor NV1FGF on amputation and death: a randomised placebo-controlled trial of gene therapy in critical limb ischaemia. Lancet 377, 1929-1937 (2011).

27. Niagara, M. I. et al. Autologous skeletal myoblasts transduced with a new adenoviral bicistronic vector for treatment of hind limb ischemia. J. Vasc. Surg. 40, 774-785 (2004).

28. Furmston, J., Patel, A. \& et al. Angiogenic cell therapy for critical limb ischemia: an update on concepts and trials. J. Cardiovasc. Surg. (Torino). 55, 641-54 (2014).

29. Walter, D. H. et al. Intraarterial Administration of Bone Marrow Mononuclear Cells in Patients With Critical Limb Ischemia: A Randomized-Start, Placebo-Controlled Pilot Trial (PROVASA). Circ. Cardiovasc. Interv. 4, 26-37 (2011).

30. Idei, N. \& et al. Autologous Bone-Marrow Mononuclear Cell Implantation Reduces Long-Term Major Amputation Risk in Patients With Critical Limb Ischemia. Circ. Cardiovasc. Interv. 4, 14 25 (2011).

31. Powell, R. J. et al. Interim analysis results from the RESTORE-CLI, a randomized, double-blind multicenter phase II trial comparing expanded autologous bone marrow-derived tissue repair cells and placebo in patients with critical limb ischemia. J. Vasc. Surg. 54, 1032-1041 (2011).

32. Teraa, M., Sprengers, R. W., Schutgens, R. E. G., Tweel, I. Van Der \& Doevendans, P. A. Effect of Repetitive Intra-Arterial Infusion of Bone Marrow Mononuclear Cells in Patients with No- 
Option Limb Ischemia. Cardiovasc. Surg. 131, 851-60 (2015).

33. M, T. \& et al. Autologous bone marrow-derived cell therapy in patients with critical limb ischemia: a meta-analysis of randomized controlled clinical trials. Ann Surg 258, 922-929 (2013).

34. Weem, S. M. O. P., Teraa, M. \& Borst, G. J. De. Bone Marrow derived Cell Therapy in Critical Limb Ischemia: A Meta-analysis of Randomized Placebo Controlled Trials. Eur. J. Vasc. Endovasc. Surg. 50, 775-783 (2015).

35. Tateishi-Yuyama, E. et al. Therapeutic angiogenesis for patients with limb ischaemia by autologous transplantation of bone-marrow cells: a pilot study and a randomised controlled trial. Lancet 360, 427-435 (2002).

36. Pittenger, M. F. et al. Multilineage Potential of Adult Human Mesenchymal Stem Cells. Science (80-. ). 284, 143-148 (1999).

37. Iwase, T., Nagaya, N., Fujii, T., Itoh, T. \& Murakami, S. Comparison of angiogenic potency between mesenchymal stem cells and mononuclear cells in a rat model of hindlimb ischemia. Cardiovasc. Res. 66, 543-551 (2005).

38. Meyer, G. P. et al. Intracoronary Bone Marrow Cell Transfer After Myocardial Infarction: Eighteen Months' Follow-Up Data From the Randomized, Controlled BOOST (BOne marrOw transfer to enhance ST-elevation infarct regeneration) Trial. Circulation 113, 1287-94 (2006).

39. Benoit, E. et al. The role of amputation as an outcome measure in cellular therapy for critical limb ischemia: implications for clinical trial design. J. Transl. Med. 9, (2011).

40. Iafrati, M. D. et al. Early results and lessons learned from a multicenter, randomized, double-blind trial of bone marrow aspirate concentrate in critical limb ischemia. J. Vasc. Surg. 54, 1650-1658 (2011).

41. Hjortholm, N. \& Jaddini, E. Strategies of pain reduction during the bone marrow biopsy. Ann. Hematol. 92, 145-149 (2013).

42. Dong, Z., Chen, B., Fu, W., Wang, Y. \& Guo, D. Transplantation of purified CD34+ cells in the treatment of critical limb ischemia. J. Vasc. Surg. 58, 404-411 (2011).

43. Kawamoto, A. \& et al. Intramuscular Transplantation of G-CSF-Mobilized CD34+ Cells in Patients With Critical Limb Ischemia: A Phase I/IIa, Multicenter, Single-Blinded, Dose-Escalation Clinical Trial. Stem Cells 27, 2857-2864 (2009).

44. Kinoshita, M., Fujita, Y., Katayama, M., Baba, R. \& Shibakawa, M. Long-term clinical outcome after intramuscular transplantation of granulocyte colony stimulating factor-mobilized CD34 positive cells in patients with critical limb ischemia. Atherosclerosis 224, 440-445 (2012).

45. Losordo, D. \& et al. A randomized controlled pilot study of autologous CD34+ cell therapy for critical limb ischemia. Circ. Cardiovasc. Interv. 5, 821-830 (2013).

46. Murphy, T. P. et al. Supervised Exercise Versus Primary Stenting for Claudication Resulting From Aortoiliac Peripheral Artery Disease Six-Month Outcomes From the Claudication: Exercise Versus Endoluminal Revascularization (CLEVER) Study. Circulation 125, 130-9 (2012). 
47. Fakhry, F., Rouwet, E. V, Hoed, P. T. Den, Hunink, M. G. M. \& Spronk, S. Long-term clinical effectiveness of supervised exercise therapy versus endovascular revascularization for intermittent claudication from a randomized clinical trial. Br. J. Surg. 100, 1164-1171 (2013).

48. Parmenter, B. J., Dieberg, G. \& Smart, N. A. Exercise Training for Management of Peripheral Arterial Disease : A Systematic Review and Meta-Analysis. Sport. Med. 45, 231-244 (2015).

49. Bäck, M. et al. Home-based supervised exercise versus hospital-based supervised exercise or unsupervised walk advice as treatment for intermittent claudication. $J$ Rehabil Med 47, 801-808 (2015).

50. Gey, D. C. \& et al. Management of Peripheral Arterial Disease. Am. Fam. Physician 69, 525-32 (2004).

51. Hyldahl, R. D., Olson, T., Welling, T., Groscost, L. \& Parcell, A. C. Satellite cell activity is differentially affected by contraction mode in human muscle following a work-matched bout of exercise. Front. Physiol. 5, 1-11 (2014).

52. Arnold, L. et al. Inflammatory monocytes recruited after skeletal muscle injury switch into antiinfl ammatory macrophages to support myogenesis. J. Exp. Med. 204, 1057-1069 (2007).

53. Saclier, M., Cuvellier, S. \& et al. Monocyte/macrophage interactions with myogenic precursor cells during skeletal muscle regeneration. FEBS J. 280, 4118-4130 (2013).

54. Chazaud, B. et al. Satellite cells attract monocytes and use macrophages as a support to escape apoptosis and enhance muscle growth. J. Cell Biol. 163, 1133-43 (2003).

55. Christov, C., Chre, F., Bassez, G., Gherardi, R. K. \& Marne, P. X. De. Muscle Satellite Cells and Endothelial Cells: Close Neighbors and Privileged Partners. Mol. Biol. Cell 18, 1397-1409 (2007).

56. Rhoads, R. P. et al. Satellite cell-mediated angiogenesis in vitro coincides with a functional hypoxia-inducible factor pathway. Am. J. Physiol. - Cell Physiol. 296, C1321-8 (2009).

57. Sherwood, R. I. et al. Isolation of Adult Mouse Myogenic Progenitors: Functional Heterogeneity of Cells within and Engrafting Skeletal Muscle. Cell 119, 543-554 (2004).

58. Meligy, F. Y. \& Shigemura, K. The efficiency of in vitro isolation and myogenic differentiation of MSCs derived from adipose connective tissue, bone marrow, and skeletal muscle tissue. Vitr. Cell Dev. Biol. - Anim. 48, 203-215 (2012).

59. Ding, Z. \& Huang, H. Mesenchymal stem cells in rabbit meniscus and bone marrow exhibit a similar feature but a heterogeneous multi-differentiation potential : superiority of meniscus as a cell source for meniscus repair. 1-14 (2015). doi:10.1186/s12891-015-0511-8

60. Fuchs, E., Tumbar, T. \& Guasch, G. Socializing with the Neighbors : Stem Cells and Their Niche. Cell 116, 769-778 (2004).

61. Jones, D. L. \& Wagers, A. J. No place like home: anatomy and function of the stem cell niche. Nat. Rev. Mol. Cell Biol. 9, 1-21 (2008).

62. Alessandri, G. et al. Isolation and culture of human muscle-derived stem cells able to differentiate 
into myogenic and neurogenic cell. Lancet 364, 1872-73 (2004).

63. Tamaki, T., Uchiyama, Y., Hirata, M., Hashimoto, H. \& Moylan, J. S. Therapeutic isolation and expansion of human skeletal muscle-derived stem cells for the use of muscle-nerve-blood vessel reconstitution. Front. Physiol. 6, (2015).

64. Nguyen, L. L. et al. Female gender and oral anticoagulants are associated with wound complications in lower extremity vein bypass: An analysis of 1404 operations for critical limb ischemia. J. Vasc. Surg. 46, 1191-1198 (2007).

65. Lo, R. C. et al. Presentation, treatment, and outcome differences between men and women undergoing revascularization or amputation for lower extremity peripheral arterial disease. $J$. Vasc. Surg. 59, 409-418 (2014).

66. Gommans, L. N. M., Scheltinga, M. R. M. \& Sambeek, M. R. H. M. Van. Gender differences following supervised exercise therapy in patients with intermittent claudication. J. Vasc. Surg. 62, 681-688 (2015).

67. Collins, T. C., Suarez-almazor, M. \& Bush, R. L. Gender and Peripheral Arterial Disease. J. Am. Board Fam. Med. 19, 132-140 (2006).

68. Brevetti, G. \& et al. Women and peripheral arterial disease: same disease, different issues. $J$. Cardiovasc. Med. 9, 382-8 (2008).

69. Nelson, W. D. et al. Sex-Dependent Attenuation of Plaque Growth After Treatment With Bone Marrow Mononuclear Cells. Circ. Res. 101, 1319-27 (2007).

70. Yetkin, E., Topal, E. \& Erguzel, N. Diabetes mellitus and female gender are the strongest predictors of poor collateral vessel development in patients with severe coronary artery stenosis. Angiogenesis 18, 201-207 (2015).

71. Sigvant, B., Wiberg-hedman, K. \& Bergqvist, D. A population-based study of peripheral arterial disease prevalence with special focus on critical limb ischemia and sex differences. J. Vasc. Surg. 45, 1185-1191 (2007).

72. Teodorescu, V. J., Vavra, A. K., Kibbe, M. R. \& York, N. Peripheral arterial disease in women. J. Vasc. Surg. 57, 18S-26S (2013).

73. Bruce, A. C. et al. Monocytes are recruited from venules during arteriogenesis in the murine spinotrapezius ligation model. Arterioscler. Thromb. Vasc. Biol. 34, 2012-2022 (2014).

74. Lee, K. Y. \& Mooney, D. J. Hydrogels for Tissue Engineering. Chem. Rev. 101, 1869-79 (2001).

75. Bruce, A. C. \& Peirce, S. M. Exogenous Thrombin Delivery Promotes Collateral Capillary Arterialization and Tissue Reperfusion in the Murine Spinotrapezius Muscle Ischemia Model. Microcirculation 19, 143-154 (2012).

76. Shahid, M. \& Nunhuck, A. Physiology. (Mosby, 2008).

77. Arras, M. et al. Monocyte activation in angiogenesis and collateral growth in the rabbit hindlimb. J. Clin. Invest. 101, 40-50 (1998). 
78. Fung, E. \& Helisch, A. Macrophages in collateral arteriogenesis. Front. Physiol. 24, (2012).

79. Heil, M. et al. Collateral Artery Growth (Arteriogenesis) After Experimental Arterial Occlusion is Impaired in Mice Lacking CC-Chemokine Receptor-2. Circ. Res. 94, 671-7 (2004).

80. Price, R. J., Owens, G. K. \& Skalak, T. C. Immunohistochemical Identification of Arteriolar Development Using Markers of Smooth Muscle Differentiation Evidence That Capillary Arterialization Proceeds From Terminal Arterioles. Circ. Res. 75, 520-527 (1994).

81. Westvik, T. S., Fitzgerald, T. N. \& Muto, A. Limb ischemia after iliac ligation in aged mice stimulates angiogenesis without arteriogenesis. J. Vasc. Surg. 49, 464-473 (2009).

82. Weel, V. Van et al. More Dominantly Than Hyperglycemia or Insulin Resistance in Mice. Arterioscler. Thromb. Vasc. Biol. 26, 1383-1390 (2006).

83. Arruda, R. M. P., Peotta, V. A., Meyrelles, S. S. \& Vasquez, E. C. Evaluation of Vascular Function in Apolipoprotein E Knockout Mice With Angiotensin-Dependent Renovascular Hypertension. Hypertension 46, 932-937 (2005).

84. Cola, M. S., Gava, A. L., Meyrelles, S. S. \& Vasquez, E. C. Endothelial dysfunction of resistance vessels in female apolipoprotein E-deficient mice. Lipids Health Dis. 9, 1-5 (2010).

85. Zhou, X., Teng, B. \& Mustafa, S. J. Sex Difference in Coronary Endothelial Dysfunction in Apolipoprotein E Knockout Mouse: Role of NO and A2A Adenosine Receptor. Microcirculation 22, 518-27 (2015).

86. Caligiuri, G., Nicoletti, A., Zhou, X. \& To, I. Effects of sex and age on atherosclerosis and autoimmunity in apoE-deficient mice. Atherosclerosis 145, 301-308 (1999).

87. Maeda, N. et al. Anatomical differences and atherosclerosis in apolipoprotein E-deficient mice with 129 / SvEv and C57BL / 6 genetic backgrounds. Atherosclerosis 195, 75-82 (2007).

88. Landázuri, N., Joseph, G., Guldberg, R. E. \& Taylor, W. R. Growth and regression of vasculature in healthy and diabetic mice after hindlimb ischemia. Am J Physiol Reful Integr Comp Physiol 303, R48-R56 (2012).

89. Unger, E. F. et al. Basic fibroblast growth factor enhances myocardial collateral flow in a canine model. Am. J. Physiol. 266, H1588-99 (1994).

90. Banai, S. et al. Angiogenic-Induced Enhancement of Collateral Blood Flow to Ischemic Myocardium by Vascular Endothelial Growth Factor in Dogs. Circulation 89, 2183-9 (1994).

91. Yanagisawa-miwa, A. A., Uchida, Y. \& Nakamura, F. Salvage of Infarcted Myocardium by Angiogenic Action of Basic Fibroblast Growth Factor. Science (80-. ). 257, 1401-1403 (1992).

92. Gounis, M. J. et al. Angiogenesis is confined to the transient period of VEGF expression that follows adenoviral gene delivery to ischemic muscle. Gene Ther. 12, 762-771 (2005).

93. Katsanos, K., Karnabatidis, D., Diamantopoulos, A., Siablis, D. \& Tsopanoglou, N. E. Thrombin promotes arteriogenesis and hemodynamic recovery in a rabbit hindlimb ischemia model. J. Vasc. Surg. 49, 1000-1012 (2009). 
94. Fu, X., Wang, H., Hu, P., Rna, E. Á. N. \& Dna, Á. Stem cell activation in skeletal muscle regeneration. Cell. Mol. Life Sci. 72, 1663-1677 (2015).

95. Formigli, L. et al. Paracrine effects of transplanted myoblasts and relaxin on post-infarction heart remodelling. J Cell Mol Med 11, 1087-1100 (2007).

96. Ren, B. et al. ERK 1/2-Akt1 crosstalk regulates arteriogenesis in mice and zebrafish. J. Clin. Invest. 120, 1217-28 (2010).

97. Kofler, N. M. \& Simons, M. Angiogenesis versus arteriogenesis : neuropilin 1 modulation of VEGF signaling. F1000 Prime Reports 7, 1-7 (2015).

98. Schaper, W. Collateral Circulation Past and Present. Basic Res. Cardiol. 104, 5-21 (2009).

99. Meisner, J. K., Sumer, S., Murrell, K. P., Higgins, T. J. \& Price, R. J. Laser Speckle Flowmetry Method for Measuring Spatial and Temporal Hemodynamic Alterations Throughout Large Microvascular Networks. Microcirculation 19, 619-631 (2012).

100. Scholz, D. et al. Ultrastructure and molecular histology of rabbit hind-limb collateral artery growth (arteriogenesis). Virchows Arch 436, 257-270 (2000).

101. Khmelewski, E., Becker, A., Meinertz, T. \& Ito, W. D. Tissue Resident Cells Play a Dominant Role in Arteriogenesis and Concomitant Macrophage Accumulation. Circ. Res. 95, 56-65 (2004).

102. Liu, Y., Zou, X., Chai, Y. \& Yao, Y. Macrophage Polarization in Inflammatory Diseases. Int. J. Biol. Sci. 10, 520-529 (2014).

103. Takeda, Y. \& Costa, S. Macrophage skewing by Phd2 haplodeficiency prevents ischaemia by inducing arteriogenesis. Nature 479, 122-6 (2011).

104. Troidl, C. et al. The Temporal and Spatial Distribution of Macrophage Subpopulations During Arteriogenesis. Curr. Vasc. Pharmacol. 11, 5-12 (2013).

105. Tietze, E. Unpublished.

106. Go, J. Influence of Myoblasts on Arteriogenesis in a Murine Chronic Hindlimb Ischemia Model. (2014).

107. Allen, A. B., Gazit, Z., Su, S., Stevens, H. Y. \& Guldberg, R. E. In Vivo Bioluminescent Tracking of Mesenchymal Stem Cells Within Large Hydrogel Constructs. Tissue Eng. Part C 20, 806-816 (2014).

108. Ren, K., He, C., Xiao, C., Li, G. \& Chen, X. Injectable glycopolypeptide hydrogels as biomimetic scaffolds for cartilage tissue engineering. Biomaterials 51, 238-249 (2015).

109. Chan, B. P. \& W, L. K. Scaffolding in tissue engineering : general approaches and tissue-specific considerations. Eur Spine J 17, S467-79 (2008).

110. Mccain, M. L., Agarwal, A., Nesmith, H. W., Nesmith, A. P. \& Parker, K. K. Micromolded Gelatin Hydrogels for Extended Culture of Engineered Cardiac Tissues. Biomaterials 35, 54625471 (2015). 
111. Jarquin-Yanez, K. Structural Effect of Different EDC Crosslinker Concentration in GelatinHyaluronic Acid Scaffolds. J. Bioeng. Biomed. Sci. 6, (2016).

112. Ali, S., Wall, I. B., Mason, C., Pelling, A. E. \& Veraitch, F. S. The effect of Young's modulus on the neuronal differentiation of mouse embryonic stem cells. Acta Biomater. 25, 253-267 (2015).

113. Ofner, C. \& Bubnis, W. Chemical and Swelling Evaluations of Amino Group Crosslinking in Gelatin and Modified Gelatin Matrices. Pharm. Res. 13, 1821-27 (1996).

114. Liang, H., Chang, W., Liang, H., Lee, M. \& Sung, H. Crosslinking Structures of Gelatin Hydrogels Crosslinked with Genipin or a Water-Soluble Carbodiimide. J. Appl. Polym. Sci. 91, 4017-26 (2004).

115. Bidarra, S. J., Barrias, C. C. \& Granja, P. L. Injectable alginate hydrogels for cell delivery in tissue engineering. Acta Biomater. 10, 1646-1662 (2014).

116. Kuo, C. K. \& Ma, P. X. Ionically crosslinked alginate hydrogels as scaffolds for tissue engineering:Part 1. Structure, gelation rate and mechanical properties. Biomaterials 22, 511-521 (2001).

117. Tahrir, F. G. \& et al. Injectable Thermosensitive Chitosan/Clycerophosphate-Based Hydrogels for Tissue Engineering and Drug Delivery Applications. Recent Pat. Drug Deliv. Formul. 9, 107-120 (2015).

118. Aguado, B. A. et al. Improving Viability of Stem Cells During Syringe Needle Flow Through the Design of Hydrogel Cell Carriers. Tissue Eng. Part A 18, 806-815 (2012). 


\section{APPENDICES \\ Appendix A: Surgery and Experimental Protocols Spinotrapezius Ligation Protocol}

Date

Mouse Information

DOB:

Sex:

Tag:

Genotype/strain

Cage:

Weight:

Materials

Instruments

1. Standard Pattern Forceps (1)

2. Iris Scissors (1)

3. $\mathrm{S} \& \mathrm{~T}(1)$

4. $5 / 45(1)$

5. Dumont \#7's (1)

6. Microdissection scissors (1)

7. Castro Viejos (1)

Pre-sterilize in autoclave

8. Cotton gauze (4)

9. Cotton swabs (4)

10. 6.0 silk suture $(2 \times 1$ in $)$

11. 7-0 Prolene Suture

12. Surgical Drape

Obtained in surgery suite

13. Petri dish w/ sterile saline

14. Gloves (non-sterile and sterile)

15. FST heat pad $\mathrm{w} /$ rectal probe

16. Recovery bin and weigh boat

17. Depilatory cream

18. non-sterile cotton swabs

19. non-sterile cotton gauze

20. Isolation mask \& cap

21. Analgesic (buprenorphine)

\section{Surgery Preparation}

22. Spray surgery area with Nolvasan

23. Weigh animal in weight boat

24. Place animal in anesthesia box

25 . Open the oxygen cylinder and set anesthesiamachine flow meter to $\sim 31 \cdot \mathrm{min}^{-1}$

26. Anesthetize animal w/ $5 \%$ isoflurane

27. Reduce flow rate to $0.5-1.01 \cdot \mathrm{min}^{-1}$ and the isoflurane to $1-3 \%$

28. Lay animal prone with nose in nose-cone

29. Remove hair on anterior dorsal aspect of the animal with clippers and depilatory cream.

30. Apply vetinary ointment to eyes to avoid drying during the procedure

31. Give animal subcutaneous injection of
Spinotrapezius Ligation Initials

buprenorphine

32. Lay animal prone on heat pad (w/4 $\mathrm{x} 4$ on top) w/ nose in nose-cone

33. Insert rectal probe and set thermo-controller to $35^{\circ} \mathrm{C}$

34. Adjust focus, lighting, and center mouse

35. Don isolation mask, cap, and scrubs

36. Open sterile surgical instrument pack

37. Open sterile pack and dump in surgical instrument field

38. Place sterile petri dish in sterile field and fill with sterile saline

39. Don sterile gloves

40. Place surgical drape on the animal and arrange the items in sterile field

Surgery

41. Make $5 \mathrm{~mm}$ incision parallel to spine at intersection of fat pad and muscle

42. Dissect skin overlying the spinotrapezius muscle

43. Search for lateral feed artery vein pair jumping from the underlying fat pad to the spinotrapezius

44. Blunt dissect fat pad to reveal arteriole-venule pair, using paparvrine as a vasodilator if necessary

45. Blunt dissect arteriole away from venule

46. Pass a separated strand from 6-0 silk suture below the arteriole and tie off

47. Repeat again about $1 \mathrm{~mm}$ above previous ligature

48. Cut arteriole between the two ligatures with micro dissection scissors

49. Suture incision with 7-0 prolene suture

50. Repeat for contralateral side to perform sham, but do not tie off ligature

Post-Surgical

51. Give the animal a subcutaneous injection of buprenorphine

52. Place the animal in the recovery bin, on a blue bench cover, above a heat pad and allow to recover

53. Turn the flow meter down to 0 , turn off the isoflurane, and close the oxygen cylinder

54 . Indicate surgery on cage card

\section{Notes}

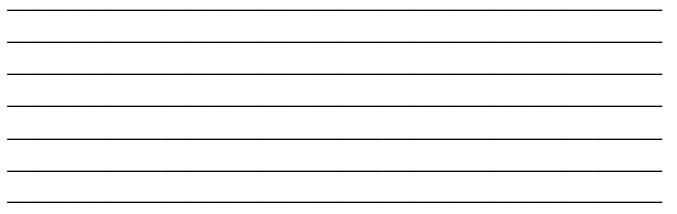




\section{Sterile Gelatin Preparation Protocol}

Date:

Initials:

\section{Sterile Materials}

- $25 \mathrm{ml}$ Erlenmeyer flask

- Rubber stopper + tubing

- Rubber stopper

- Spatula

- Distilled $\mathrm{H}_{2} \mathrm{O}$

- Gelatin powder

\section{Non-Sterile Materials}

- Hot plate

- Analytical balance

- 6mM 1-Ethyl-3-(3-dimethylaminopropyl) carbodiimide (EDC)
- $10 \mathrm{~mL}$ syringe

- Syringe filter

- Pipettes

- $50 \mathrm{~mL}$ conicals

- 24 Well Plate

\section{Procedure}

1. Turn hot plate on to $200^{\circ} \mathrm{C}$

2. In the hood, open the sterile packs with the Erlenmeyer flask and the rubber stopper

3. Weigh empty $25 \mathrm{ml}$ Erlenmeyer flask + rubber stopper on an analytical balance and zero the balance

4. Bring the Erlenmeyer flask back to the hood, and bring the gelatin into the hood

5. Open the sterile pack with the spatula and use to put some gelatin powder into the Erlenmeyer flask

6. Put the rubber stopper back on the flask and weigh it on the analytical balance

7. Add enough distilled $\mathrm{H}_{2} \mathrm{O}$ to the flask to make a $10 \%$ gelatin solution (by weight)

8. Open the sterile pack with the rubber stopper + tubing and put it on the flask

9. Bring the flask to the hot, wait for it to reach a boil, and then allow it to boil for 5 minutes

10. Bring the flask back into the hood and put $0.3 \mathrm{~mL}$ of the gelatin solution in each well

11. While the gelatin in solidifying use the syringe filters to filter the $6 \mathrm{mM}$ EDC

12. Once the gelatin has solidified, add $0.5 \mathrm{~mL}$ of EDC to each well

13. Leave the gelatin in the EDC solution overnight at $4{ }^{\circ} \mathrm{C}$ to allow for crosslinking

14. The next day aspirate the EDC solution and wash the gel $3 \times 30$ minutes with sterile $\mathrm{H}_{2} \mathrm{O}$ 
Development of Gelatin Boiling Technique

We tried a couple techniques for sealing and venting the gelatin solution before developing the final solution. The first attempt utilized a foam stopper in an Erlenmeyer flask, but this method did not allow sufficient ventilation. The pressure buildup in the flask prevented the foam stopper from sealing the flask throughout the entire boiling process. The second attempt was to use a side arm distilling head and a round bottom flask borrowed from the chemistry department, which successfully sealed the flask and allowed ventilation. However, during the glassware testing a simpler solution was developed, so we decided to continue with the simpler solution for the final protocol. This solution used an Erlenmeyer flask with a vented rubber stopper and a piece of curved tubing attached with a tubing connector (Figure 19).

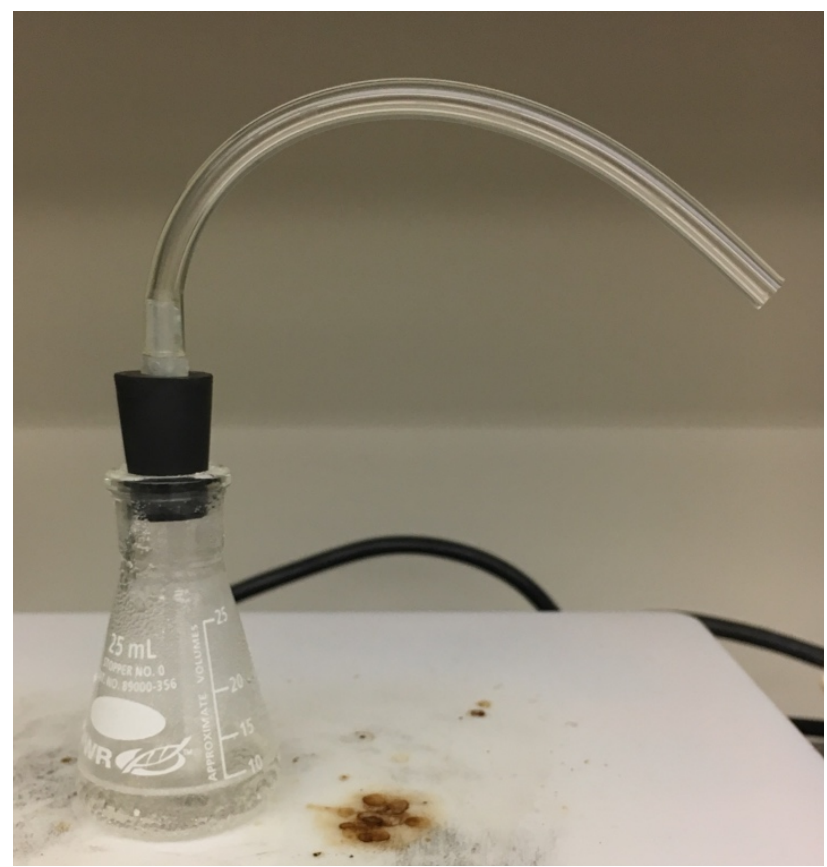

Figure 19. Preparation of sterile gelatin vehicles. Sterile gelatin was prepared by weighing gelatin powder in a cell culture hood and adding sterile water to make a $10 \%$ gelatin solution. The solution was then boiled outside the hood while sealed and vented, as shown, and aliquoted into a 24-well plate to solidify. 


\section{Myoblast Passing Protocol}

Date:

Primary Myoblast Expansion Protocol

Initials:

Purpose: To expand primary mouse myoblasts from live Extensor Digitorum (EDL) muscle fibers

\section{Necessary Materials:}

- Growth Media (20\% FBS in Base Media)

- SB 203580 working solution (10 mmol)

- $\quad$ bFGF working solution $(1 \mu \mathrm{g} / \mathrm{ml}$ in PBS)

- Cell Dissociation Solution - EDTA (Fisher 13151014)

- Trypsin/EDTA (0.05\% Invitrogen 25300-062)

- $\operatorname{PBS}\left(-/-, \mathrm{Ca}^{+2}, \mathrm{Mg}^{+2}\right)$

- ECM coated flask

1. __ Obtain an $\sim 80 \%$ confluent culture vessel

a. myoblasts will begin to differentiate and form myotubes as they approach confluency

2. Clean Phase Contrast Microscope with $70 \% \mathrm{v} / \mathrm{v}$ Isopropanol (IPA)

3. Image save pictures of the culture with a phase contrast microscope

4. Determine passage ratio to be used

a. myoblasts should not be split at a ratio higher than 1:3

5. ___ Choose passaging method based upon goals and culture conditions

a. use Trypsin for:

i. for better cell distribution, single cell suspension

ii. for a confluent flask with low overall confluency (1:1 pass)

b. use EDTA for:

i. preservation of surface receptors (integrins)

Trypsin Passaging

ii. selective passaging (more differentiated cells will more strongly adhere)

1. Warm Trypsin and Growth Media in $37 \mathrm{C} \mathrm{H}_{2} \mathrm{O}$ bath immediately before use

2. _ Aspirate media from culture vessel

3. W_ Wash with PBS (-/-) and aspirate

4. __ Add warm trypsin

a. $\quad 0.04 \mathrm{ml} / \mathrm{cm}^{2}$ surface (T12.5 - $\left.0.5 \mathrm{ml}, \mathrm{T} 75-3 \mathrm{ml}\right)$

5. __ Immediately place in incubator for $\sim 2 \mathrm{~min}$

6. __ Observe with phase contrast microscope

a. cells should begin to ball up and become phase bright

7. Hit the flask repeatedly to dislodge weakly adherent cells

8. _ Observe under the phase contrast microscope to ensure detachment of all cells

a. If cells are still attached, return them to the incubator for an additional min and repeat

9. Add the same volume of Growth Media as trypsin to stop enzymatic cleavage

10. Wash the surface of the culture vessel with the cell suspension

11. Label a $15 \mathrm{ml}$ conical tube and transfer the cell suspension

12. Centrifuge at $300 \mathrm{~g}$ for $5 \mathrm{~min}$ to create a cell pellet

13. Aspirate media from the conical

a. be careful to avoid aspirating the pellet

14.

15.

16.

17. Resuspend in the volume of growth media (+ factors) appropriate for the chosen passage ratio Repeatedly pipette up and down to break of cell clumps

Rock the culture vessel repeatedly: forward/back and left/right to evenly disperse the cells

a. Even cell distribution can be checked under the phase contrast microscope

Place in incubator

a. close the door gently to prevent uneven cell distribution 


\section{Perfusion Fixation Protocol}

Date

\section{Mouse Information}

DOB:

Sex

Tag:

Genotype/strain:

Cage:

Weight(g)

\section{Materials}

Non-Sterilize Dissection Instruments

1. Forceps (1)

2. Fine forceps (2)

3. Bone scissors (1)

4. Curved Iris scissors (1)

5. Microdissection scissors (1)

6. Vascular clamp (1)

7. Castroviejos

Obtained in surgery suite

\begin{tabular}{|c|c|}
\hline 8. & Tape \\
\hline 9. & $20 \mathrm{~mL}$ syringes $(2)$ \\
\hline 10. & $5 \mathrm{~mL}$ syringe (1) \\
\hline 11. & Syringe pump \\
\hline 12. & Petri-dish \\
\hline 13. & Bench cover \\
\hline 14. & Depilatory cream \\
\hline 15. & Clippers \\
\hline 16. & Veterinary ointment \\
\hline 17. & Heating pad \\
\hline 18. & Catheter \\
\hline 19. & Non-sterile saline \\
\hline 20. & Cotton swabs \\
\hline 21. & Gauze sponges \\
\hline 22. & Saran wrap \\
\hline
\end{tabular}

\section{Vasodilator Cocktail Preparation}

23. Turn on water bath to $37^{\circ} \mathrm{C}$

24. $400 \mu \mathrm{L}$ heparin

25. $1 \mathrm{~mL} \mathrm{SNP(orange)}$

26. $600 \mu \mathrm{L}$ Adenosine(clear)

27. $38 \mathrm{~mL}$ PBS solution

28. $5 \mathrm{~mL} \mathrm{4 \%} \mathrm{Paraformaldehyde} \mathrm{(PFA)}$

29. Thaw SNP, Adenosine and PFA

30. Add heparin, SNP, Adenosine, and PBS solution together in a $50 \mathrm{~mL}$ conical

31. Place vasodilator cocktail in water bath

\section{Procedure Preparation}

32. Obtain saline filled petri-dish, cotton swab, and instruments
Perfusion Fixation

Initials

Fixation

33. Remove hair on back by shaving \& depilation

34. Expose muscles of interest and blunt dissect to aid in removal post-fixation, then cover with saran wrap

35. Tape animal in supine position to $4 \mathrm{X} 4$ gauze sponge over heating pad

36. Fill $10 \mathrm{~mL}$ syringe with $10 \mathrm{~mL}$ warm Vaso D, load into syringe pump and attach catheter

37. Flow liquid through the catheter to the tip to prevent air from being injected into circulatory system

38. Lift skin from muscle in abdominal region and cut a window over the sternum

39. Lift sternum and cut connective tissue under

40. Use bone scissors to quickly cut through the ribs to the armpit on both sides

41. Clamp sternum with castroviejos and reflect towards mouse's head

42. Cut diaphragm with curved iris scissors to open chest cavity

43. Cut away excess tissue around the heart

44. Make a small incision in the apex of the heart

45. Insert catheter and clamp with vascular clamp and cut right atrium

46. Inject Vaso D solution into animal approximately $4 \times 10 \mathrm{~mL}(2 \mathrm{~mL} / \mathrm{min}$, $14.43 \mathrm{~mm}$ diameter), soaking up excess blood and fluids with gauze sponges

47. Inject $5 \mathrm{~mL} \mathrm{PFA} \mathrm{(1} \mathrm{mL/min,} 11.99 \mathrm{~mm}$ diameter)

48. Dissect out muscles of interest using fine forceps and microdissection scissors

49. Turn off water bath, cover scope, turn off oxygen, turn off isofluorane, and clean instruments 


\section{Staining Protocol}

Date:

Alpha-Smooth Muscle Actin, Lectin, and CD68 Staining Protocol

Initials:

\section{Materials}

- 24-well culture plates (Cat\#: 3738, Corning Incorporated)

- $\mathrm{PBS}$

- $\quad 0.1 \%$ Triton-X (Cat\#:T8787, Sigma-Aldrich)

- $2 \%$ Bovine Serum Albumin (Cat\# B6917, Sigma Aldrich)

- Monoclonal Anti-Alpha Smooth Muscle Actin, Cy3 Conjugate (Cat\#: C6198, Sigma-Aldrich)

- Isolectin GS-IB4 from GRI DyLight 649 (Cat \#: DL-1208, Vector Laboratories)

- Rat Anti Mouse CD68 FA-11 Clone Alexa Fluor 488 (Cat \#: MCA1957A488T, Bio-Rad)

- Glass Slides

- Glass Coverslips

- Parafilm

- Nail Polish

- Aluminum foil

\section{Staining}

1. Using forceps, remove muscle from PBS (stored in microcentrifuge tube at $4^{\circ} \mathrm{C}$ ) and place in a single well of a 24 -well culture plate.

2. Prepare antibody solution containing 1:200 1A4 clone (alpha-smooth muscle actin, Cy3 conjugate), 1:100 Isolectin GS-IB4 from GRI (Alexa Fluor 647), and 1:200 Rat Anti Mouse CD68 FA-11 Clone (Alexa Fluor 488) in $0.1 \%$ Triton-X, 2\% BSA (reconstituted in PBS) in PBS, using $0.3 \mathrm{~mL}$ of solution per muscle.

3. Wrap the 24-well plate in parafilm and incubate muscle in antibody solution at $4{ }^{\circ} \mathrm{C}$ for 72 hours for the Spinotrapezius or 7 days for the Gracilis.

4. Wash in $0.1 \%$ Triton- $x$ in PBS $3 x$ for 20 minutes at room temperature. Cover plate with foil during each wash.

5. Wash in plain PBS for 30 minutes at room temperature. Cover with foil during wash.

6. Place 1-2 drops of 50/50 PBS and Glycerol onto slide.

7. Remove muscle from well using forceps and place on a slide. Depression slides are best for the gracilis because the muscle is thick and the coverslip will not lie flat on a flat slide.

8. Add 1-2 drops of 50/50 PBS and Glycerol to the top of the muscle and place coverslip over the muscle.

9. Paint edges of coverslip with clear nail polish to create a seal and prevent tissue desiccation.

10. Store slides at $4{ }^{\circ} \mathrm{C}$ wrapped in foil or an opaque container between imaging.

11. Wait 24 hours before imaging to allow the glycerol to clear and the nail polish to dry.

\section{Imaging}

12. Image between three arteriolar trees using the $20 \mathrm{x}$ objective on the confocal microscope.

13. Use the following lasers: Alexa Fluor 488 for CD68, Alexa Fluor 559 for alpha-smooth muscle actin, Alexa Fluor 647 for lectin 
Appendix B: Sample Images

Key

\begin{tabular}{|l|l|}
\hline Stain Color & Meaning \\
\hline Red & $\alpha$-SMA vessels (ACCs) \\
\hline Blue & Lectin vessels (capillaries) \\
\hline Green & CD68 Cells (macrophages) \\
\hline
\end{tabular}

Female Ligated

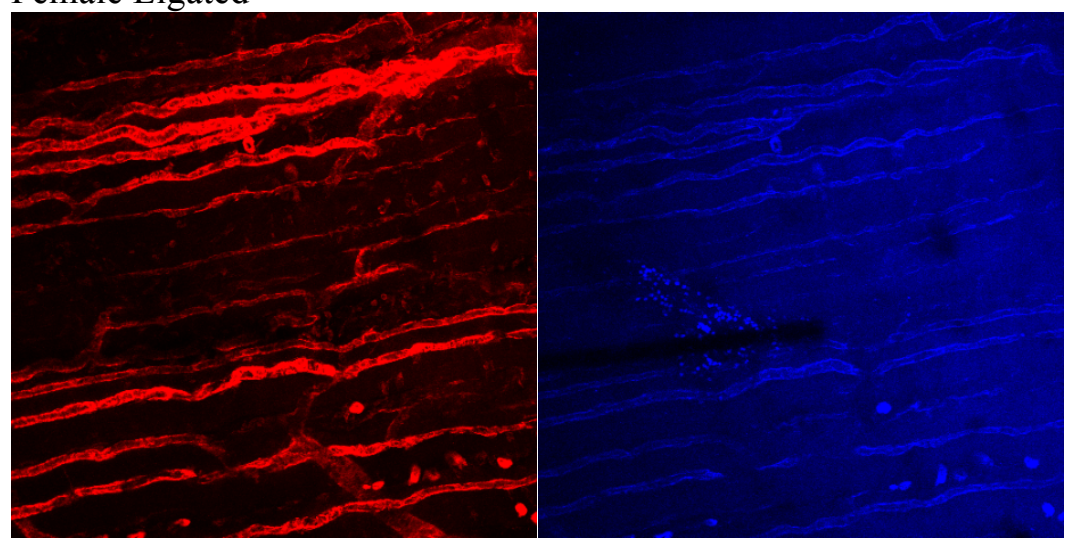

Female Sham

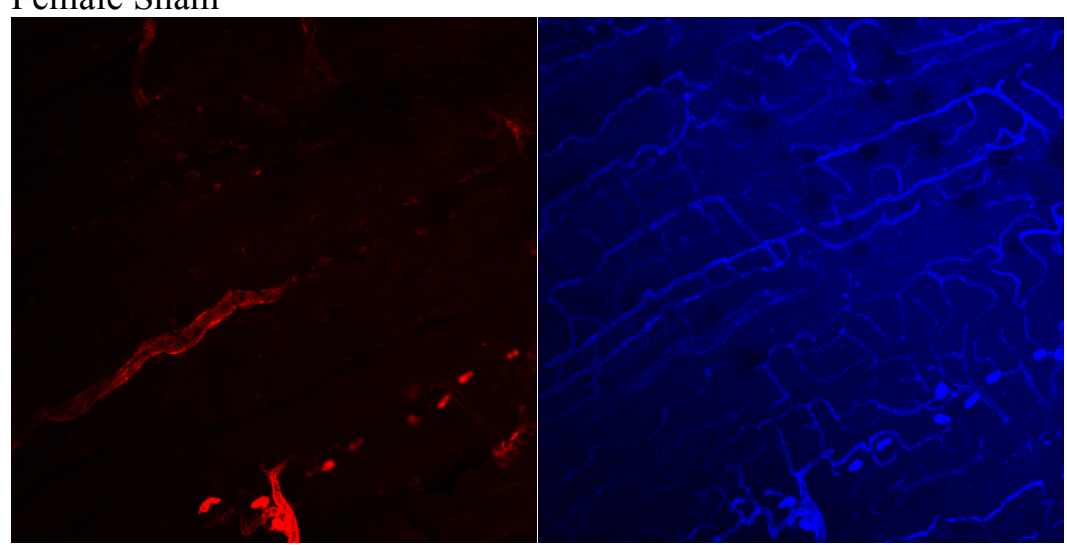

Male Ligated

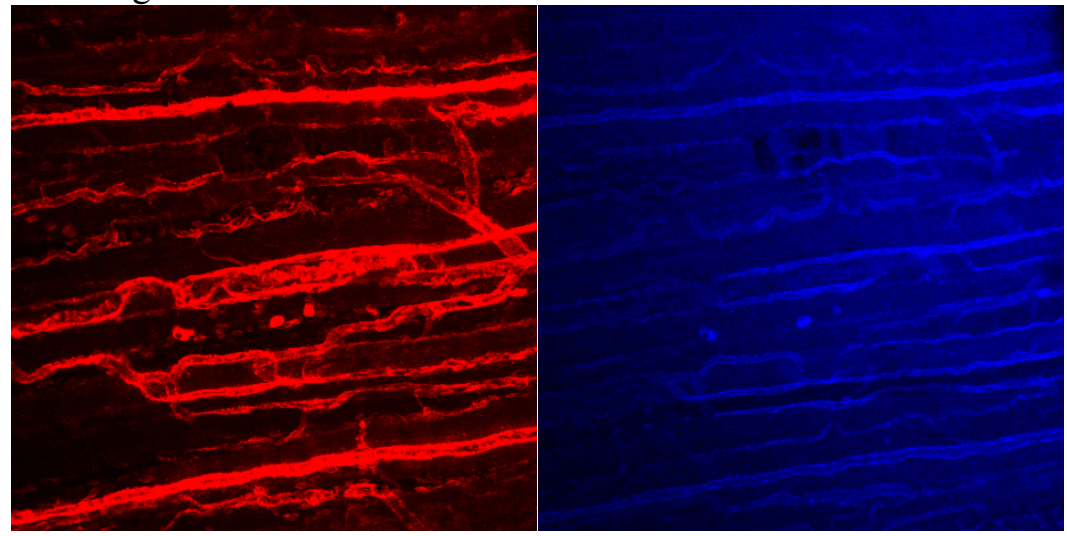


Male Sham

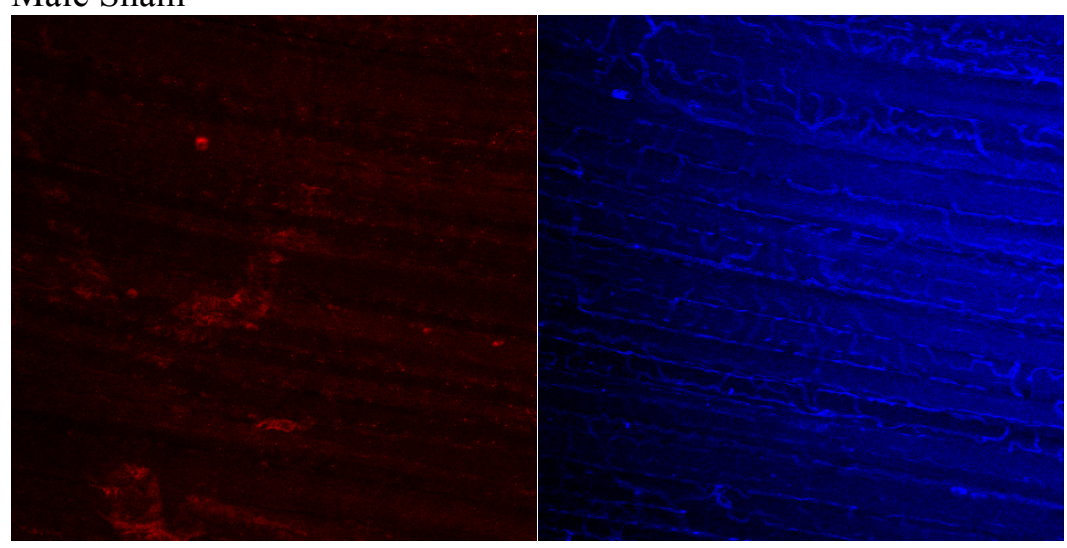

Myogenic Cells Ligated

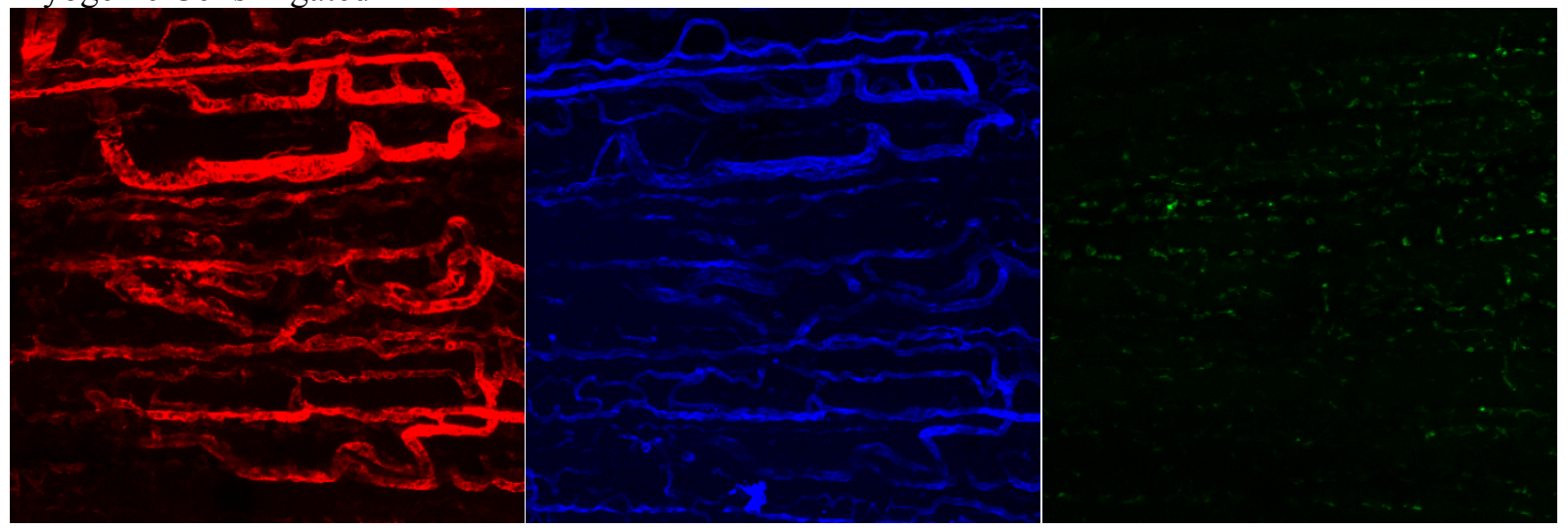

Myogenic Cells Sham

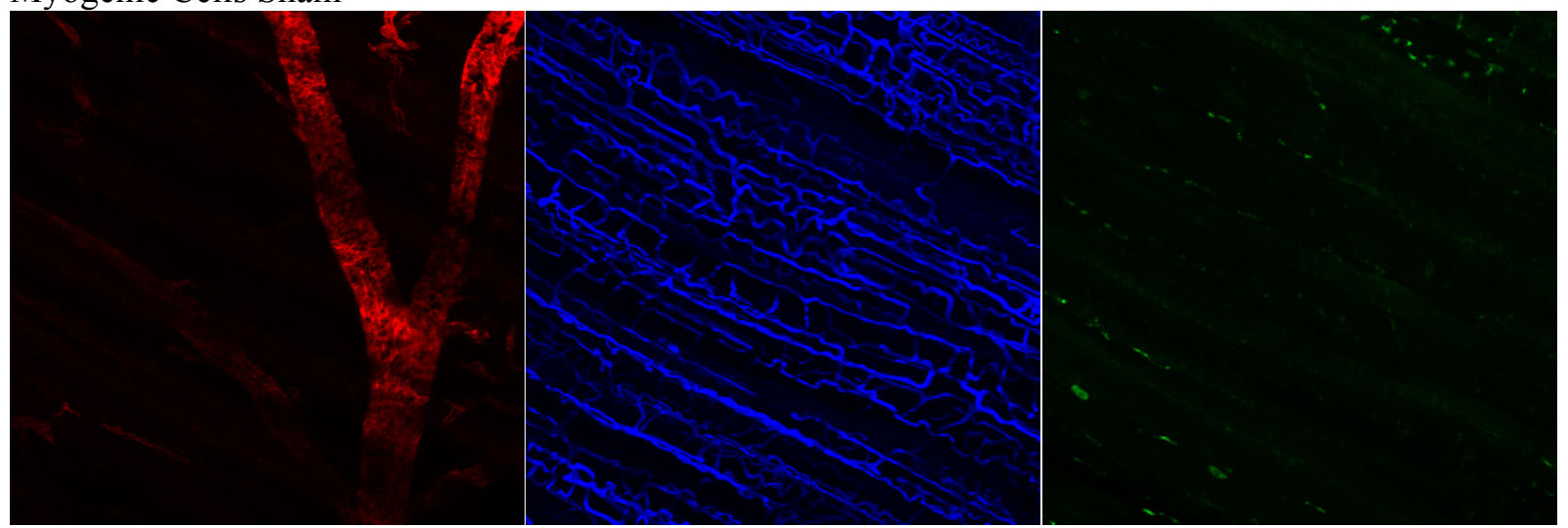


Vehicle Ligated

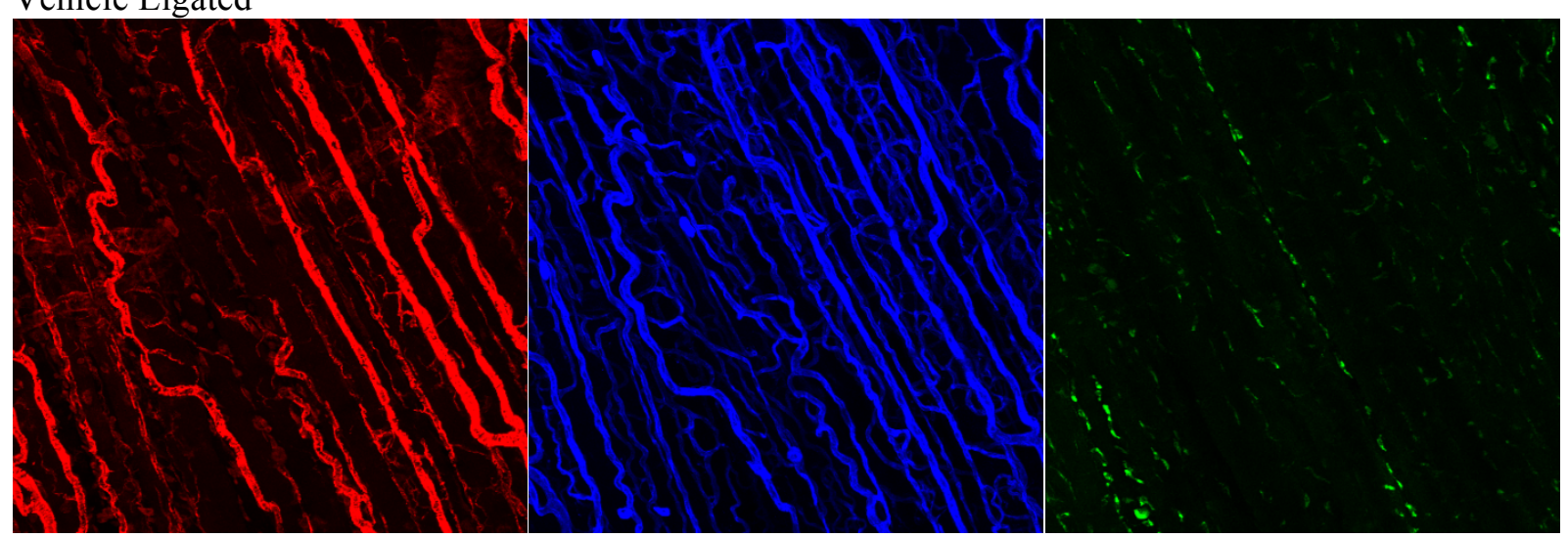

Vehicle Sham

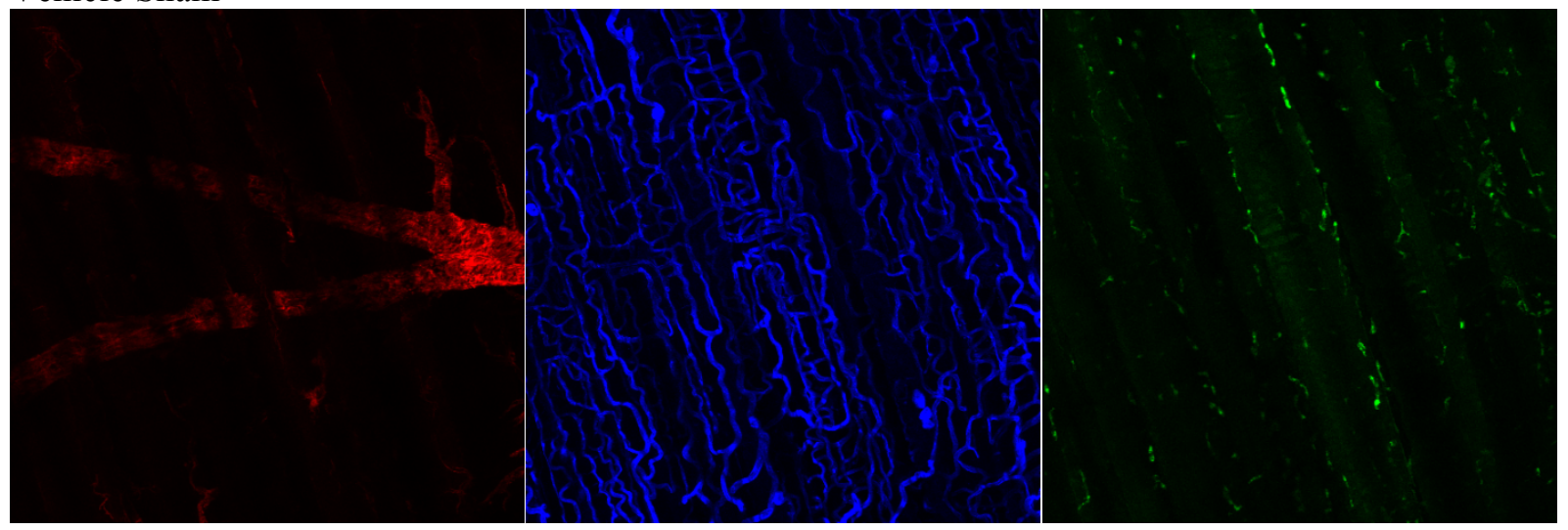

Thrombin Ligated

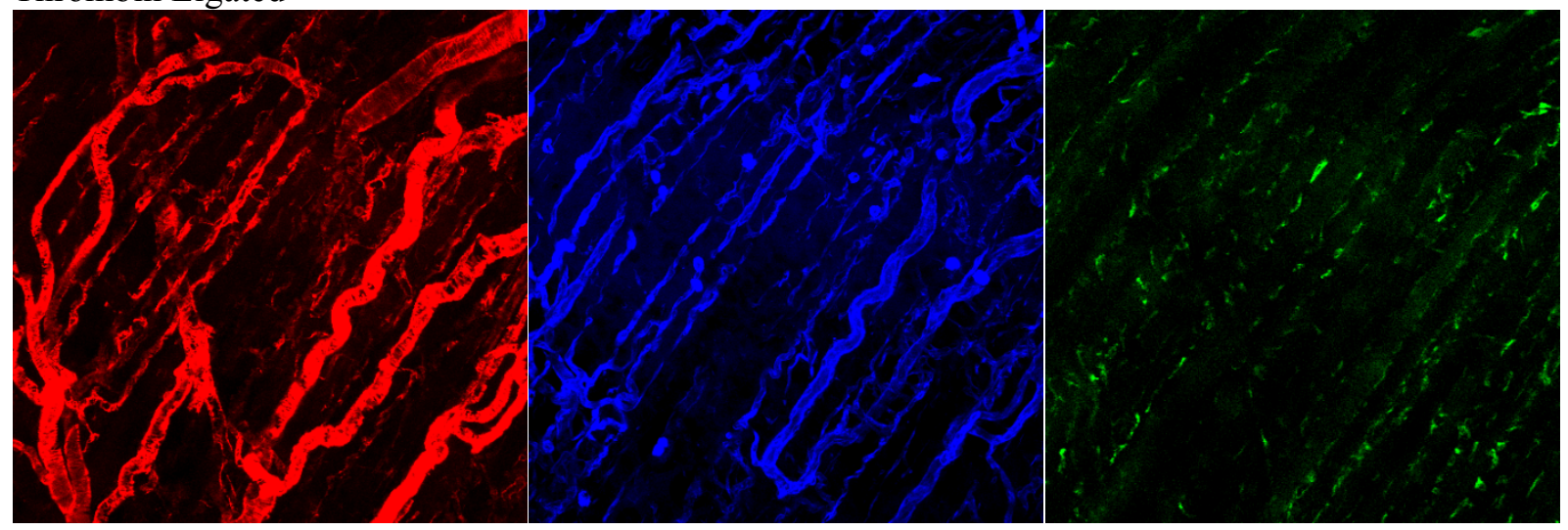




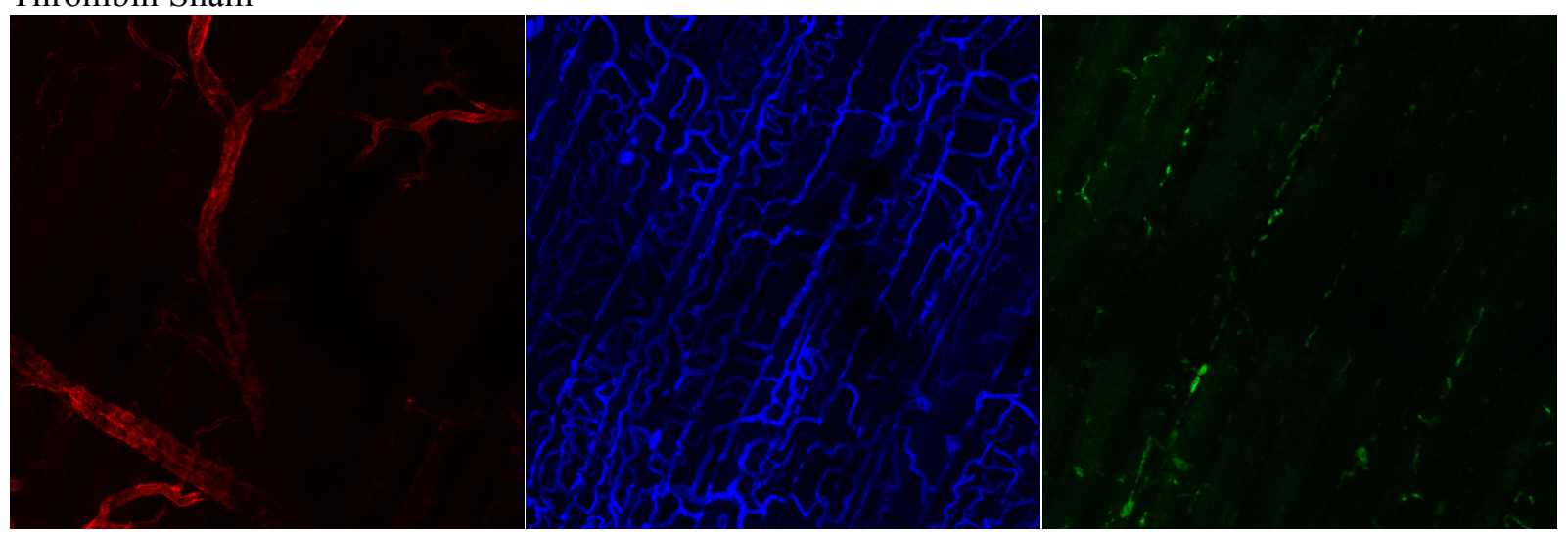

\section{Ligation Only Ligated}

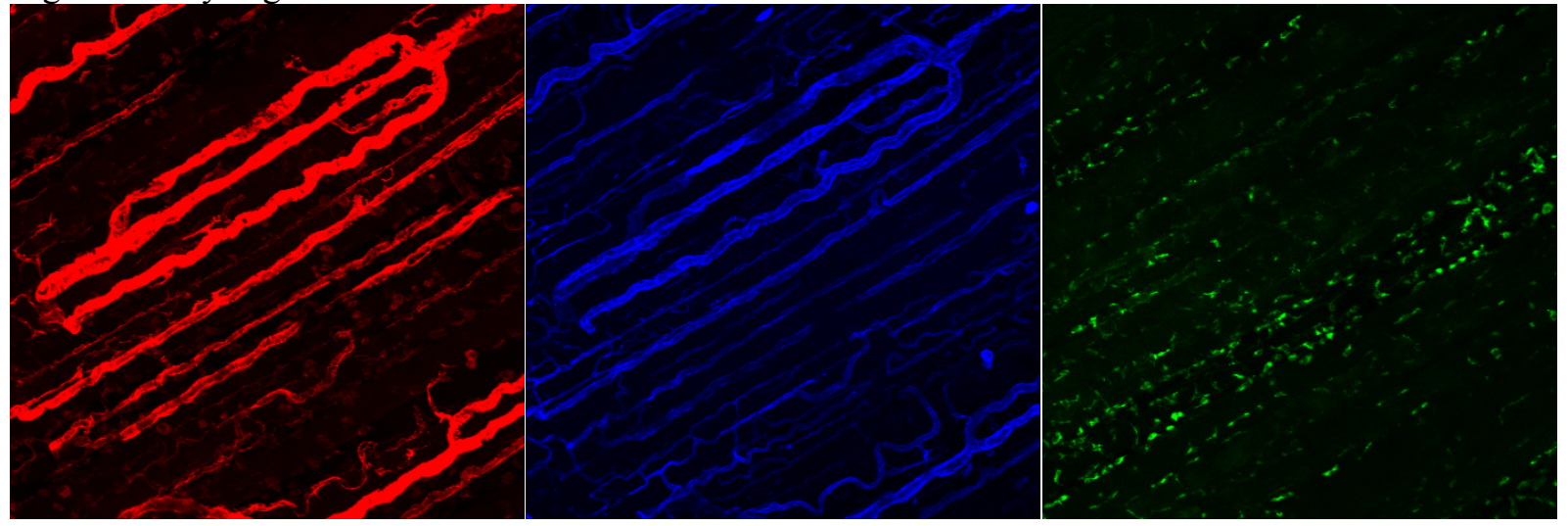

Ligation Only Sham

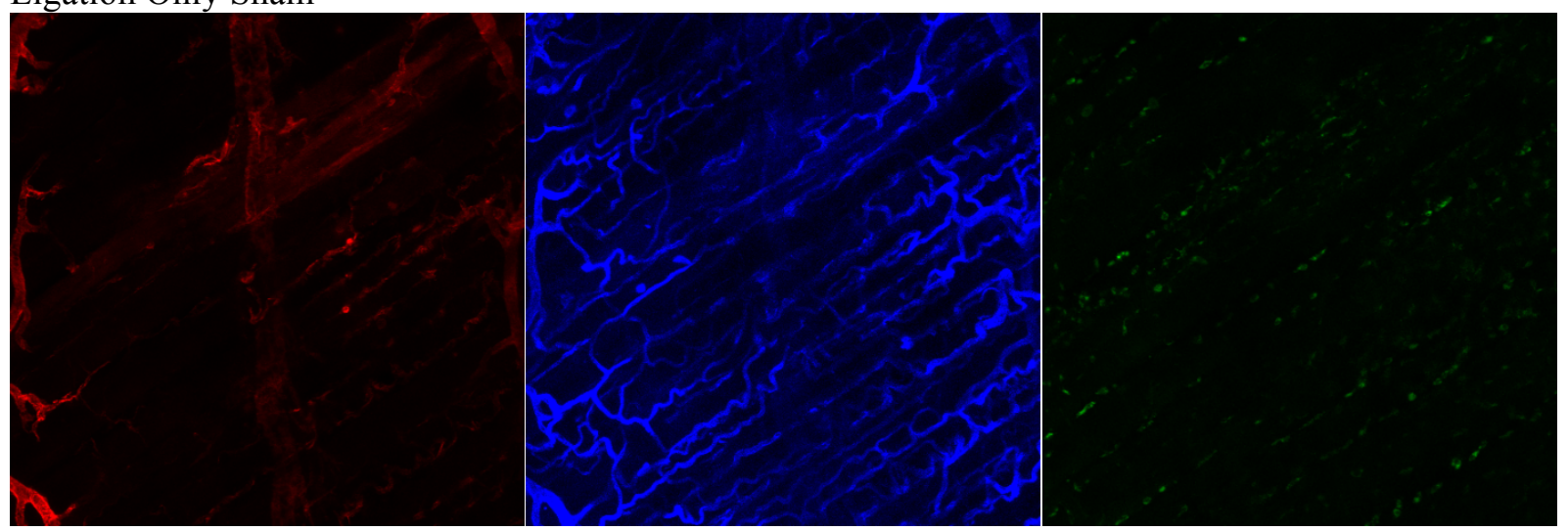


Appendix C: Raw Data

Male and Female Vessel Count Data

\begin{tabular}{|c|c|c|c|c|c|c|}
\hline Replicate & Sex & Muscle & Region & $\begin{array}{c}\text { Total } \\
\text { Vessels } \\
\end{array}$ & Capillaries & $\begin{array}{l}\alpha \text {-SMA } \\
\text { Vessels } \\
\end{array}$ \\
\hline 1 & Male & Ligated & Region 1 & PEC & PEC & PEC \\
\hline 1 & Male & Ligated & Region 2 & PEC & PEC & PEC \\
\hline 1 & Male & Ligated & Region 3 & PEC & PEC & PEC \\
\hline 2 & Male & Ligated & Region 1 & 9 & 4 & 5 \\
\hline 2 & Male & Ligated & Region 2 & 10 & 6 & 4 \\
\hline 2 & Male & Ligated & Region 3 & 10 & 4 & 5 \\
\hline 3 & Male & Ligated & Region 1 & 12 & 7 & 5 \\
\hline 3 & Male & Ligated & Region 2 & 10 & 5 & 5 \\
\hline 3 & Male & Ligated & Region 3 & 8 & 3 & 5 \\
\hline 4 & Male & Ligated & Region 1 & 11 & 6 & 5 \\
\hline 4 & Male & Ligated & Region 2 & 11 & 7 & 4 \\
\hline 4 & Male & Ligated & Region 3 & 10 & 6 & 4 \\
\hline 5 & Male & Ligated & Region 1 & 17 & 12 & 5 \\
\hline 5 & Male & Ligated & Region 2 & 17 & 12 & 5 \\
\hline 5 & Male & Ligated & Region 3 & 15 & 10 & 5 \\
\hline 6 & Male & Ligated & Region 1 & 13 & 8 & 5 \\
\hline 6 & Male & Ligated & Region 2 & 13 & 8 & 5 \\
\hline 6 & Male & Ligated & Region 3 & 14 & 9 & 5 \\
\hline 7 & Male & Ligated & Region 1 & 10 & 4 & 6 \\
\hline 7 & Male & Ligated & Region 2 & 10 & 5 & 5 \\
\hline 7 & Male & Ligated & Region 3 & 9 & 3 & 6 \\
\hline 8 & Male & Ligated & Region 1 & PEC & PEC & PEC \\
\hline 8 & Male & Ligated & Region 2 & PEC & PEC & PEC \\
\hline 8 & Male & Ligated & Region 3 & PEC & PEC & PEC \\
\hline 1 & Male & Sham & Region 1 & 10 & 10 & 0 \\
\hline 1 & Male & Sham & Region 2 & 12 & 12 & 0 \\
\hline 1 & Male & Sham & Region 3 & 10 & 10 & 0 \\
\hline 2 & Male & Sham & Region 1 & 10 & 10 & 0 \\
\hline 2 & Male & Sham & Region 2 & 8 & 8 & 0 \\
\hline 2 & Male & Sham & Region 3 & 10 & 10 & 0 \\
\hline 3 & Male & Sham & Region 1 & 10 & 10 & 0 \\
\hline 3 & Male & Sham & Region 2 & 10 & 10 & 0 \\
\hline 3 & Male & Sham & Region 3 & 12 & 12 & 0 \\
\hline
\end{tabular}




\begin{tabular}{|c|c|c|c|c|c|c|}
\hline 4 & Male & Sham & Region 1 & 16 & 16 & 0 \\
\hline 4 & Male & Sham & Region 2 & 9 & 9 & 0 \\
\hline 4 & Male & Sham & Region 3 & 12 & 12 & 0 \\
\hline 5 & Male & Sham & Region 1 & 12 & 12 & 0 \\
\hline 5 & Male & Sham & Region 2 & 19 & 19 & 0 \\
\hline 5 & Male & Sham & Region 3 & 14 & 14 & 0 \\
\hline 6 & Male & Sham & Region 1 & 10 & 10 & 0 \\
\hline 6 & Male & Sham & Region 2 & 15 & 15 & 0 \\
\hline 6 & Male & Sham & Region 3 & 12 & 12 & 0 \\
\hline 7 & Male & Sham & Region 1 & 16 & 16 & 0 \\
\hline 7 & Male & Sham & Region 2 & 9 & 9 & 0 \\
\hline 7 & Male & Sham & Region 3 & 11 & 11 & 0 \\
\hline 8 & Male & Sham & Region 1 & 14 & 14 & 0 \\
\hline 8 & Male & Sham & Region 2 & 11 & 11 & 0 \\
\hline 8 & Male & Sham & Region 3 & 11 & 11 & 0 \\
\hline 1 & Female & Ligated & Region 1 & PEC & PEC & PEC \\
\hline 1 & Female & Ligated & Region 2 & PEC & PEC & PEC \\
\hline 1 & Female & Ligated & Region 3 & PEC & PEC & PEC \\
\hline 2 & Female & Ligated & Region 1 & 12 & 7 & 5 \\
\hline 2 & Female & Ligated & Region 2 & 10 & 6 & 4 \\
\hline 2 & Female & Ligated & Region 3 & 9 & 5 & 4 \\
\hline 3 & Female & Ligated & Region 1 & 9 & 4 & 5 \\
\hline 3 & Female & Ligated & Region 2 & 10 & 6 & 4 \\
\hline 3 & Female & Ligated & Region 3 & 13 & 8 & 5 \\
\hline 4 & Female & Ligated & Region 1 & PEC & PEC & PEC \\
\hline 4 & Female & Ligated & Region 2 & PEC & PEC & PEC \\
\hline 4 & Female & Ligated & Region 3 & PEC & PEC & PEC \\
\hline 5 & Female & Ligated & Region 1 & 11 & 7 & 4 \\
\hline 5 & Female & Ligated & Region 2 & 11 & 6 & 5 \\
\hline 5 & Female & Ligated & Region 3 & 12 & 7 & 5 \\
\hline 6 & Female & Ligated & Region 1 & PEC & PEC & PEC \\
\hline 6 & Female & Ligated & Region 2 & PEC & PEC & PEC \\
\hline 6 & Female & Ligated & Region 3 & PEC & PEC & PEC \\
\hline 7 & Female & Ligated & Region 1 & PEC & PEC & PEC \\
\hline 7 & Female & Ligated & Region 2 & PEC & PEC & PEC \\
\hline 7 & Female & Ligated & Region 3 & PEC & PEC & PEC \\
\hline 8 & Female & Ligated & Region 1 & 10 & 5 & 5 \\
\hline 8 & Female & Ligated & Region 2 & 11 & 7 & 4 \\
\hline 8 & Female & Ligated & Region 3 & 11 & 6 & 5 \\
\hline
\end{tabular}




\begin{tabular}{|l|l|l|l|l|l|l|}
\hline 1 & Female & Sham & Region 1 & 12 & 12 & 0 \\
\hline 1 & Female & Sham & Region 2 & 9 & 9 & 0 \\
\hline 1 & Female & Sham & Region 3 & 11 & 11 & 0 \\
\hline 2 & Female & Sham & Region 1 & 10 & 10 & 0 \\
\hline 2 & Female & Sham & Region 2 & 10 & 10 & 0 \\
\hline 2 & Female & Sham & Region 3 & 11 & 11 & 0 \\
\hline 3 & Female & Sham & Region 1 & 12 & 12 & 0 \\
\hline 3 & Female & Sham & Region 2 & 11 & 11 & 0 \\
\hline 3 & Female & Sham & Region 3 & & & 0 \\
\hline 4 & Female & Sham & Region 1 & 11 & 11 & 0 \\
\hline 4 & Female & Sham & Region 2 & 13 & 13 & 0 \\
\hline 5 & Female & Sham & Region 3 & 14 & 14 & 0 \\
\hline 5 & Female & Sham & Region 1 & 12 & 12 & 0 \\
\hline 5 & Female & Sham & Region 2 & 11 & 11 & 0 \\
\hline 6 & Female & Sham & Region 3 & 11 & 11 & 0 \\
\hline 6 & Female & Sham & Region 2 & 9 & 9 & 0 \\
\hline 6 & Female & Sham & Region 3 & 12 & 12 & 0 \\
\hline
\end{tabular}

PEC indicates that the muscle had a pre-existing collateral and measurements were not taken 
Male and Female Vessel Diameter Data

\begin{tabular}{|c|c|c|c|c|c|c|}
\hline Replicate & Sex & Muscle & Region & Vessel & $\begin{array}{l}\alpha-S M A \text { Vessel } \\
\text { Diameter }(\mu \mathrm{m})\end{array}$ & $\begin{array}{c}\text { Lectin Vessel } \\
\text { Diameter }(\mu \mathrm{m})\end{array}$ \\
\hline 1 & Male & Ligated & Region 1 & Vessel 1 & PEC & PEC \\
\hline 1 & Male & Ligated & Region 1 & Vessel 2 & PEC & PEC \\
\hline 1 & Male & Ligated & Region 1 & Vessel 3 & PEC & PEC \\
\hline 1 & Male & Ligated & Region 2 & Vessel 1 & PEC & PEC \\
\hline 1 & Male & Ligated & Region 2 & Vessel 2 & PEC & PEC \\
\hline 1 & Male & Ligated & Region 2 & Vessel 3 & PEC & PEC \\
\hline 1 & Male & Ligated & Region 3 & Vessel 1 & PEC & PEC \\
\hline 1 & Male & Ligated & Region 3 & Vessel 2 & PEC & PEC \\
\hline 1 & Male & Ligated & Region 3 & Vessel 3 & PEC & PEC \\
\hline 2 & Male & Ligated & Region 1 & Vessel 1 & 12.5 & 6.1 \\
\hline 2 & Male & Ligated & Region 1 & Vessel 2 & 12.6 & 4.8 \\
\hline 2 & Male & Ligated & Region 1 & Vessel 3 & 10.2 & 4.4 \\
\hline 2 & Male & Ligated & Region 2 & Vessel 1 & 14.8 & \\
\hline 2 & Male & Ligated & Region 2 & Vessel 2 & 12.0 & \\
\hline 2 & Male & Ligated & Region 2 & Vessel 3 & 8.5 & \\
\hline 2 & Male & Ligated & Region 3 & Vessel 1 & 12.1 & 4.6 \\
\hline 2 & Male & Ligated & Region 3 & Vessel 2 & 10.8 & 4.7 \\
\hline 2 & Male & Ligated & Region 3 & Vessel 3 & 10.9 & 4.0 \\
\hline 3 & Male & Ligated & Region 1 & Vessel 1 & 9.4 & 3.8 \\
\hline 3 & Male & Ligated & Region 1 & Vessel 2 & 7.5 & 3.9 \\
\hline 3 & Male & Ligated & Region 1 & Vessel 3 & 10.8 & 5.3 \\
\hline 3 & Male & Ligated & Region 2 & Vessel 1 & 9.7 & 4.7 \\
\hline 3 & Male & Ligated & Region 2 & Vessel 2 & 12.1 & 4.3 \\
\hline 3 & Male & Ligated & Region 2 & Vessel 3 & 12.4 & 4.1 \\
\hline 3 & Male & Ligated & Region 3 & Vessel 1 & 11.4 & 4.6 \\
\hline 3 & Male & Ligated & Region 3 & Vessel 2 & 9.8 & 4.5 \\
\hline 3 & Male & Ligated & Region 3 & Vessel 3 & 8.6 & 4.2 \\
\hline 4 & Male & Ligated & Region 1 & Vessel 1 & 11.8 & 4.4 \\
\hline 4 & Male & Ligated & Region 1 & Vessel 2 & 7.2 & 3.8 \\
\hline 4 & Male & Ligated & Region 1 & Vessel 3 & 11.1 & 4.1 \\
\hline 4 & Male & Ligated & Region 2 & Vessel 1 & 10.9 & 4.5 \\
\hline 4 & Male & Ligated & Region 2 & Vessel 2 & 10.6 & 3.1 \\
\hline 4 & Male & Ligated & Region 2 & Vessel 3 & 11.2 & 5.1 \\
\hline 4 & Male & Ligated & Region 3 & Vessel 1 & 11.2 & 5.4 \\
\hline 4 & Male & Ligated & Region 3 & Vessel 2 & 13.0 & 4.4 \\
\hline
\end{tabular}




\begin{tabular}{|c|c|c|c|c|c|c|}
\hline 4 & Male & Ligated & Region 3 & Vessel 3 & 13.4 & 5.0 \\
\hline 5 & Male & Ligated & Region 1 & Vessel 1 & 11.6 & 4.5 \\
\hline 5 & Male & Ligated & Region 1 & Vessel 2 & 12.5 & 4.7 \\
\hline 5 & Male & Ligated & Region 1 & Vessel 3 & 6.9 & 4.5 \\
\hline 5 & Male & Ligated & Region 2 & Vessel 1 & 8.8 & 4.5 \\
\hline 5 & Male & Ligated & Region 2 & Vessel 2 & 8.6 & 4.8 \\
\hline 5 & Male & Ligated & Region 2 & Vessel 3 & 11.7 & 3.8 \\
\hline 5 & Male & Ligated & Region 3 & Vessel 1 & 15.3 & 4.1 \\
\hline 5 & Male & Ligated & Region 3 & Vessel 2 & 6.7 & 3.6 \\
\hline 5 & Male & Ligated & Region 3 & Vessel 3 & 12.5 & 4.8 \\
\hline 6 & Male & Ligated & Region 1 & Vessel 1 & 10.7 & 4.8 \\
\hline 6 & Male & Ligated & Region 1 & Vessel 2 & 14.0 & 5.0 \\
\hline 6 & Male & Ligated & Region 1 & Vessel 3 & 14.2 & 4.9 \\
\hline 6 & Male & Ligated & Region 2 & Vessel 1 & 10.9 & 3.7 \\
\hline 6 & Male & Ligated & Region 2 & Vessel 2 & 9.9 & 5.3 \\
\hline 6 & Male & Ligated & Region 2 & Vessel 3 & 10.6 & 3.9 \\
\hline 6 & Male & Ligated & Region 3 & Vessel 1 & 14.9 & 3.4 \\
\hline 6 & Male & Ligated & Region 3 & Vessel 2 & 11.0 & 4.0 \\
\hline 6 & Male & Ligated & Region 3 & Vessel 3 & 15.4 & 5.4 \\
\hline 7 & Male & Ligated & Region 1 & Vessel 1 & 12.3 & 4.4 \\
\hline 7 & Male & Ligated & Region 1 & Vessel 2 & 13.5 & 5.5 \\
\hline 7 & Male & Ligated & Region 1 & Vessel 3 & 11.4 & 4.5 \\
\hline 7 & Male & Ligated & Region 2 & Vessel 1 & 9.8 & 5.3 \\
\hline 7 & Male & Ligated & Region 2 & Vessel 2 & 9.4 & 5.7 \\
\hline 7 & Male & Ligated & Region 2 & Vessel 3 & 8.0 & 6.1 \\
\hline 7 & Male & Ligated & Region 3 & Vessel 1 & 11.1 & 5.0 \\
\hline 7 & Male & Ligated & Region 3 & Vessel 2 & 14.0 & 5.3 \\
\hline 7 & Male & Ligated & Region 3 & Vessel 3 & 13.9 & 4.4 \\
\hline 8 & Male & Ligated & Region 1 & Vessel 1 & PEC & PEC \\
\hline 8 & Male & Ligated & Region 1 & Vessel 2 & PEC & PEC \\
\hline 8 & Male & Ligated & Region 1 & Vessel 3 & PEC & PEC \\
\hline 8 & Male & Ligated & Region 2 & Vessel 1 & PEC & PEC \\
\hline 8 & Male & Ligated & Region 2 & Vessel 2 & PEC & PEC \\
\hline 8 & Male & Ligated & Region 2 & Vessel 3 & PEC & PEC \\
\hline 8 & Male & Ligated & Region 3 & Vessel 1 & PEC & PEC \\
\hline 8 & Male & Ligated & Region 3 & Vessel 2 & PEC & PEC \\
\hline 8 & Male & Ligated & Region 3 & Vessel 3 & PEC & PEC \\
\hline 1 & Male & Sham & Region 1 & Vessel 1 & $\mathrm{~N} / \mathrm{A}$ & 5.3 \\
\hline 1 & Male & Sham & Region 1 & Vessel 2 & N/A & 5.2 \\
\hline
\end{tabular}




\begin{tabular}{|c|c|c|c|c|c|c|}
\hline 1 & Male & Sham & Region 1 & Vessel 3 & N/A & 4.4 \\
\hline 1 & Male & Sham & Region 2 & Vessel 1 & $\mathrm{~N} / \mathrm{A}$ & 5.5 \\
\hline 1 & Male & Sham & Region 2 & Vessel 2 & N/A & 4.9 \\
\hline 1 & Male & Sham & Region 2 & Vessel 3 & N/A & 4.8 \\
\hline 1 & Male & Sham & Region 3 & Vessel 1 & $\mathrm{~N} / \mathrm{A}$ & 5.3 \\
\hline 1 & Male & Sham & Region 3 & Vessel 2 & N/A & 5.2 \\
\hline 1 & Male & Sham & Region 3 & Vessel 3 & N/A & 5.5 \\
\hline 2 & Male & Sham & Region 1 & Vessel 1 & N/A & 8.4 \\
\hline 2 & Male & Sham & Region 1 & Vessel 2 & $\mathrm{~N} / \mathrm{A}$ & 5.9 \\
\hline 2 & Male & Sham & Region 1 & Vessel 3 & $\mathrm{~N} / \mathrm{A}$ & 6.5 \\
\hline 2 & Male & Sham & Region 2 & Vessel 1 & N/A & 6.2 \\
\hline 2 & Male & Sham & Region 2 & Vessel 2 & N/A & 6.9 \\
\hline 2 & Male & Sham & Region 2 & Vessel 3 & $\mathrm{~N} / \mathrm{A}$ & 5.7 \\
\hline 2 & Male & Sham & Region 3 & Vessel 1 & N/A & 5.8 \\
\hline 2 & Male & Sham & Region 3 & Vessel 2 & N/A & 5.1 \\
\hline 2 & Male & Sham & Region 3 & Vessel 3 & N/A & 4.7 \\
\hline 3 & Male & Sham & Region 1 & Vessel 1 & N/A & 5.1 \\
\hline 3 & Male & Sham & Region 1 & Vessel 2 & $\mathrm{~N} / \mathrm{A}$ & 4.8 \\
\hline 3 & Male & Sham & Region 1 & Vessel 3 & N/A & 6.6 \\
\hline 3 & Male & Sham & Region 2 & Vessel 1 & N/A & 7.2 \\
\hline 3 & Male & Sham & Region 2 & Vessel 2 & N/A & 7.1 \\
\hline 3 & Male & Sham & Region 2 & Vessel 3 & $\mathrm{~N} / \mathrm{A}$ & 5.2 \\
\hline 3 & Male & Sham & Region 3 & Vessel 1 & N/A & 5.5 \\
\hline 3 & Male & Sham & Region 3 & Vessel 2 & N/A & 7.0 \\
\hline 3 & Male & Sham & Region 3 & Vessel 3 & N/A & 5.7 \\
\hline 4 & Male & Sham & Region 1 & Vessel 1 & N/A & 5.8 \\
\hline 4 & Male & Sham & Region 1 & Vessel 2 & N/A & 4.4 \\
\hline 4 & Male & Sham & Region 1 & Vessel 3 & N/A & 3.7 \\
\hline 4 & Male & Sham & Region 2 & Vessel 1 & N/A & 3.8 \\
\hline 4 & Male & Sham & Region 2 & Vessel 2 & N/A & 4.1 \\
\hline 4 & Male & Sham & Region 2 & Vessel 3 & N/A & 3.1 \\
\hline 4 & Male & Sham & Region 3 & Vessel 1 & N/A & 6.0 \\
\hline 4 & Male & Sham & Region 3 & Vessel 2 & N/A & 5.1 \\
\hline 4 & Male & Sham & Region 3 & Vessel 3 & N/A & 5.4 \\
\hline 5 & Male & Sham & Region 1 & Vessel 1 & N/A & 4.8 \\
\hline 5 & Male & Sham & Region 1 & Vessel 2 & N/A & 4.0 \\
\hline 5 & Male & Sham & Region 1 & Vessel 3 & N/A & 5.8 \\
\hline 5 & Male & Sham & Region 2 & Vessel 1 & N/A & 5.8 \\
\hline 5 & Male & Sham & Region 2 & Vessel 2 & $\mathrm{~N} / \mathrm{A}$ & 4.7 \\
\hline
\end{tabular}




\begin{tabular}{|c|c|c|c|c|c|c|}
\hline 5 & Male & Sham & Region 2 & Vessel 3 & N/A & 6.9 \\
\hline 5 & Male & Sham & Region 3 & Vessel 1 & $\mathrm{~N} / \mathrm{A}$ & 7.4 \\
\hline 5 & Male & Sham & Region 3 & Vessel 2 & $\mathrm{~N} / \mathrm{A}$ & 6.6 \\
\hline 5 & Male & Sham & Region 3 & Vessel 3 & N/A & 7.6 \\
\hline 6 & Male & Sham & Region 1 & Vessel 1 & $\mathrm{~N} / \mathrm{A}$ & 4.3 \\
\hline 6 & Male & Sham & Region 1 & Vessel 2 & N/A & 5.5 \\
\hline 6 & Male & Sham & Region 1 & Vessel 3 & N/A & 6.2 \\
\hline 6 & Male & Sham & Region 2 & Vessel 1 & N/A & 6.4 \\
\hline 6 & Male & Sham & Region 2 & Vessel 2 & $\mathrm{~N} / \mathrm{A}$ & 4.4 \\
\hline 6 & Male & Sham & Region 2 & Vessel 3 & N/A & 6.8 \\
\hline 6 & Male & Sham & Region 3 & Vessel 1 & $\mathrm{~N} / \mathrm{A}$ & 6.2 \\
\hline 6 & Male & Sham & Region 3 & Vessel 2 & N/A & 5.4 \\
\hline 6 & Male & Sham & Region 3 & Vessel 3 & $\mathrm{~N} / \mathrm{A}$ & 5.0 \\
\hline 7 & Male & Sham & Region 1 & Vessel 1 & N/A & 4.7 \\
\hline 7 & Male & Sham & Region 1 & Vessel 2 & $\mathrm{~N} / \mathrm{A}$ & 4.4 \\
\hline 7 & Male & Sham & Region 1 & Vessel 3 & $\mathrm{~N} / \mathrm{A}$ & 4.7 \\
\hline 7 & Male & Sham & Region 2 & Vessel 1 & $\mathrm{~N} / \mathrm{A}$ & 5.1 \\
\hline 7 & Male & Sham & Region 2 & Vessel 2 & $\mathrm{~N} / \mathrm{A}$ & 3.6 \\
\hline 7 & Male & Sham & Region 2 & Vessel 3 & N/A & 4.3 \\
\hline 7 & Male & Sham & Region 3 & Vessel 1 & $\mathrm{~N} / \mathrm{A}$ & 4.4 \\
\hline 7 & Male & Sham & Region 3 & Vessel 2 & N/A & 5.0 \\
\hline 7 & Male & Sham & Region 3 & Vessel 3 & $\mathrm{~N} / \mathrm{A}$ & 5.3 \\
\hline 8 & Male & Sham & Region 1 & Vessel 1 & N/A & 4.9 \\
\hline 8 & Male & Sham & Region 1 & Vessel 2 & $\mathrm{~N} / \mathrm{A}$ & 4.0 \\
\hline 8 & Male & Sham & Region 1 & Vessel 3 & N/A & 4.7 \\
\hline 8 & Male & Sham & Region 2 & Vessel 1 & $\mathrm{~N} / \mathrm{A}$ & 4.5 \\
\hline 8 & Male & Sham & Region 2 & Vessel 2 & $\mathrm{~N} / \mathrm{A}$ & 4.1 \\
\hline 8 & Male & Sham & Region 2 & Vessel 3 & $\mathrm{~N} / \mathrm{A}$ & 3.7 \\
\hline 8 & Male & Sham & Region 3 & Vessel 1 & $\mathrm{~N} / \mathrm{A}$ & 4.6 \\
\hline 8 & Male & Sham & Region 3 & Vessel 2 & N/A & 4.1 \\
\hline 8 & Male & Sham & Region 3 & Vessel 3 & N/A & 5.0 \\
\hline 1 & Female & Ligated & Region 1 & Vessel 1 & PEC & PEC \\
\hline 1 & Female & Ligated & Region 1 & Vessel 2 & PEC & PEC \\
\hline 1 & Female & Ligated & Region 1 & Vessel 3 & PEC & PEC \\
\hline 1 & Female & Ligated & Region 2 & Vessel 1 & PEC & PEC \\
\hline 1 & Female & Ligated & Region 2 & Vessel 2 & PEC & PEC \\
\hline 1 & Female & Ligated & Region 2 & Vessel 3 & PEC & PEC \\
\hline 1 & Female & Ligated & Region 3 & Vessel 1 & PEC & PEC \\
\hline 1 & Female & Ligated & Region 3 & Vessel 2 & PEC & PEC \\
\hline
\end{tabular}




\begin{tabular}{|c|c|c|c|c|c|c|}
\hline 1 & Female & Ligated & Region 3 & Vessel 3 & PEC & $\mathrm{PEC}$ \\
\hline 2 & Female & Ligated & Region 1 & Vessel 1 & 9.9 & 3.3 \\
\hline 2 & Female & Ligated & Region 1 & Vessel 2 & 11.2 & 3.4 \\
\hline 2 & Female & Ligated & Region 1 & Vessel 3 & 12.4 & 4.1 \\
\hline 2 & Female & Ligated & Region 2 & Vessel 1 & 7.7 & 4.2 \\
\hline 2 & Female & Ligated & Region 2 & Vessel 2 & 7.3 & 5.2 \\
\hline 2 & Female & Ligated & Region 2 & Vessel 3 & 11.8 & 5.1 \\
\hline 2 & Female & Ligated & Region 3 & Vessel 1 & 7.8 & 5.8 \\
\hline 2 & Female & Ligated & Region 3 & Vessel 2 & 9.2 & 5.7 \\
\hline 2 & Female & Ligated & Region 3 & Vessel 3 & 10.1 & 4.7 \\
\hline 3 & Female & Ligated & Region 1 & Vessel 1 & 9.6 & 5.0 \\
\hline 3 & Female & Ligated & Region 1 & Vessel 2 & 12.3 & 4.3 \\
\hline 3 & Female & Ligated & Region 1 & Vessel 3 & 13.2 & 6.2 \\
\hline 3 & Female & Ligated & Region 2 & Vessel 1 & 12.1 & 7.5 \\
\hline 3 & Female & Ligated & Region 2 & Vessel 2 & 11.0 & 5.9 \\
\hline 3 & Female & Ligated & Region 2 & Vessel 3 & 8.3 & 5.7 \\
\hline 3 & Female & Ligated & Region 3 & Vessel 1 & 10.9 & 5.5 \\
\hline 3 & Female & Ligated & Region 3 & Vessel 2 & 13.5 & 6.0 \\
\hline 3 & Female & Ligated & Region 3 & Vessel 3 & 11.8 & 4.3 \\
\hline 4 & Female & Ligated & Region 1 & Vessel 1 & PEC & PEC \\
\hline 4 & Female & Ligated & Region 1 & Vessel 2 & PEC & PEC \\
\hline 4 & Female & Ligated & Region 1 & Vessel 3 & PEC & PEC \\
\hline 4 & Female & Ligated & Region 2 & Vessel 1 & PEC & PEC \\
\hline 4 & Female & Ligated & Region 2 & Vessel 2 & PEC & PEC \\
\hline 4 & Female & Ligated & Region 2 & Vessel 3 & PEC & PEC \\
\hline 4 & Female & Ligated & Region 3 & Vessel 1 & PEC & PEC \\
\hline 4 & Female & Ligated & Region 3 & Vessel 2 & PEC & $\mathrm{PEC}$ \\
\hline 4 & Female & Ligated & Region 3 & Vessel 3 & PEC & $\mathrm{PEC}$ \\
\hline 5 & Female & Ligated & Region 1 & Vessel 1 & 19.3 & 4.9 \\
\hline 5 & Female & Ligated & Region 1 & Vessel 2 & 16.3 & 4.5 \\
\hline 5 & Female & Ligated & Region 1 & Vessel 3 & 8.3 & 3.6 \\
\hline 5 & Female & Ligated & Region 2 & Vessel 1 & 10.4 & 3.8 \\
\hline 5 & Female & Ligated & Region 2 & Vessel 2 & 7.6 & 4.2 \\
\hline 5 & Female & Ligated & Region 2 & Vessel 3 & 10.1 & 4.9 \\
\hline 5 & Female & Ligated & Region 3 & Vessel 1 & 9.8 & 3.4 \\
\hline 5 & Female & Ligated & Region 3 & Vessel 2 & 10.9 & 3.3 \\
\hline 5 & Female & Ligated & Region 3 & Vessel 3 & 8.6 & 3.0 \\
\hline 6 & Female & Ligated & Region 1 & Vessel 1 & PEC & $\mathrm{PEC}$ \\
\hline 6 & Female & Ligated & Region 1 & Vessel 2 & PEC & PEC \\
\hline
\end{tabular}




\begin{tabular}{|c|c|c|c|c|c|c|}
\hline 6 & Female & Ligated & Region 1 & Vessel 3 & PEC & PEC \\
\hline 6 & Female & Ligated & Region 2 & Vessel 1 & PEC & PEC \\
\hline 6 & Female & Ligated & Region 2 & Vessel 2 & PEC & PEC \\
\hline 6 & Female & Ligated & Region 2 & Vessel 3 & PEC & PEC \\
\hline 6 & Female & Ligated & Region 3 & Vessel 1 & PEC & PEC \\
\hline 6 & Female & Ligated & Region 3 & Vessel 2 & PEC & PEC \\
\hline 6 & Female & Ligated & Region 3 & Vessel 3 & PEC & PEC \\
\hline 7 & Female & Ligated & Region 1 & Vessel 1 & PEC & PEC \\
\hline 7 & Female & Ligated & Region 1 & Vessel 2 & PEC & PEC \\
\hline 7 & Female & Ligated & Region 1 & Vessel 3 & PEC & PEC \\
\hline 7 & Female & Ligated & Region 2 & Vessel 1 & PEC & PEC \\
\hline 7 & Female & Ligated & Region 2 & Vessel 2 & PEC & PEC \\
\hline 7 & Female & Ligated & Region 2 & Vessel 3 & PEC & PEC \\
\hline 7 & Female & Ligated & Region 3 & Vessel 1 & PEC & PEC \\
\hline 7 & Female & Ligated & Region 3 & Vessel 2 & PEC & PEC \\
\hline 7 & Female & Ligated & Region 3 & Vessel 3 & PEC & PEC \\
\hline 8 & Female & Ligated & Region 1 & Vessel 1 & 13.5 & 4.5 \\
\hline 8 & Female & Ligated & Region 1 & Vessel 2 & 12.8 & 4.6 \\
\hline 8 & Female & Ligated & Region 1 & Vessel 3 & 12.7 & 4.3 \\
\hline 8 & Female & Ligated & Region 2 & Vessel 1 & 12.8 & 4.8 \\
\hline 8 & Female & Ligated & Region 2 & Vessel 2 & 8.6 & 4.1 \\
\hline 8 & Female & Ligated & Region 2 & Vessel 3 & 12.0 & 5.3 \\
\hline 8 & Female & Ligated & Region 3 & Vessel 1 & 9.6 & 4.7 \\
\hline 8 & Female & Ligated & Region 3 & Vessel 2 & 6.6 & 5.6 \\
\hline 8 & Female & Ligated & Region 3 & Vessel 3 & 6.4 & 5.1 \\
\hline 1 & Female & Sham & Region 1 & Vessel 1 & N/A & 5.0 \\
\hline 1 & Female & Sham & Region 1 & Vessel 2 & N/A & 4.5 \\
\hline 1 & Female & Sham & Region 1 & Vessel 3 & $\mathrm{~N} / \mathrm{A}$ & 4.7 \\
\hline 1 & Female & Sham & Region 2 & Vessel 1 & $\mathrm{~N} / \mathrm{A}$ & 6.4 \\
\hline 1 & Female & Sham & Region 2 & Vessel 2 & $\mathrm{~N} / \mathrm{A}$ & 4.3 \\
\hline 1 & Female & Sham & Region 2 & Vessel 3 & N/A & 6.1 \\
\hline 1 & Female & Sham & Region 3 & Vessel 1 & $\mathrm{~N} / \mathrm{A}$ & 5.0 \\
\hline 1 & Female & Sham & Region 3 & Vessel 2 & N/A & 5.2 \\
\hline 1 & Female & Sham & Region 3 & Vessel 3 & $\mathrm{~N} / \mathrm{A}$ & 5.2 \\
\hline 2 & Female & Sham & Region 1 & Vessel 1 & $\mathrm{~N} / \mathrm{A}$ & 4.4 \\
\hline 2 & Female & Sham & Region 1 & Vessel 2 & $\mathrm{~N} / \mathrm{A}$ & 5.2 \\
\hline 2 & Female & Sham & Region 1 & Vessel 3 & N/A & 5.8 \\
\hline 2 & Female & Sham & Region 2 & Vessel 1 & $\mathrm{~N} / \mathrm{A}$ & 5.8 \\
\hline 2 & Female & Sham & Region 2 & Vessel 2 & N/A & 6.9 \\
\hline
\end{tabular}




\begin{tabular}{|c|c|c|c|c|c|c|}
\hline 2 & Female & Sham & Region 2 & Vessel 3 & N/A & 4.6 \\
\hline 2 & Female & Sham & Region 3 & Vessel 1 & N/A & 6.0 \\
\hline 2 & Female & Sham & Region 3 & Vessel 2 & N/A & 5.2 \\
\hline 2 & Female & Sham & Region 3 & Vessel 3 & N/A & 5.5 \\
\hline 3 & Female & Sham & Region 1 & Vessel 1 & $\mathrm{~N} / \mathrm{A}$ & 4.2 \\
\hline 3 & Female & Sham & Region 1 & Vessel 2 & N/A & 3.4 \\
\hline 3 & Female & Sham & Region 1 & Vessel 3 & N/A & 4.5 \\
\hline 3 & Female & Sham & Region 2 & Vessel 1 & N/A & 4.5 \\
\hline 3 & Female & Sham & Region 2 & Vessel 2 & N/A & 6.4 \\
\hline 3 & Female & Sham & Region 2 & Vessel 3 & N/A & 5.2 \\
\hline 4 & Female & Sham & Region 1 & Vessel 1 & $\mathrm{~N} / \mathrm{A}$ & 5.7 \\
\hline 4 & Female & Sham & Region 1 & Vessel 2 & N/A & 5.6 \\
\hline 4 & Female & Sham & Region 1 & Vessel 3 & $\mathrm{~N} / \mathrm{A}$ & 5.5 \\
\hline 4 & Female & Sham & Region 2 & Vessel 1 & N/A & 4.7 \\
\hline 4 & Female & Sham & Region 2 & Vessel 2 & $\mathrm{~N} / \mathrm{A}$ & 6.7 \\
\hline 4 & Female & Sham & Region 2 & Vessel 3 & N/A & 6.1 \\
\hline 4 & Female & Sham & Region 3 & Vessel 1 & $\mathrm{~N} / \mathrm{A}$ & 4.4 \\
\hline 4 & Female & Sham & Region 3 & Vessel 2 & N/A & 4.5 \\
\hline 4 & Female & Sham & Region 3 & Vessel 3 & N/A & 5.5 \\
\hline 5 & Female & Sham & Region 1 & Vessel 1 & N/A & 4.5 \\
\hline 5 & Female & Sham & Region 1 & Vessel 2 & N/A & 5.5 \\
\hline 5 & Female & Sham & Region 1 & Vessel 3 & N/A & 5.0 \\
\hline 5 & Female & Sham & Region 2 & Vessel 1 & N/A & 5.3 \\
\hline 5 & Female & Sham & Region 2 & Vessel 2 & $\mathrm{~N} / \mathrm{A}$ & 5.4 \\
\hline 5 & Female & Sham & Region 2 & Vessel 3 & N/A & 4.6 \\
\hline 5 & Female & Sham & Region 3 & Vessel 1 & N/A & 7.0 \\
\hline 5 & Female & Sham & Region 3 & Vessel 2 & N/A & 5.7 \\
\hline 5 & Female & Sham & Region 3 & Vessel 3 & $\mathrm{~N} / \mathrm{A}$ & 6.3 \\
\hline 6 & Female & Sham & Region 1 & Vessel 1 & N/A & 5.1 \\
\hline 6 & Female & Sham & Region 1 & Vessel 2 & $\mathrm{~N} / \mathrm{A}$ & 4.9 \\
\hline 6 & Female & Sham & Region 1 & Vessel 3 & N/A & 5.3 \\
\hline 6 & Female & Sham & Region 2 & Vessel 1 & N/A & 4.8 \\
\hline 6 & Female & Sham & Region 2 & Vessel 2 & N/A & 4.7 \\
\hline 6 & Female & Sham & Region 2 & Vessel 3 & $\mathrm{~N} / \mathrm{A}$ & 5.2 \\
\hline 6 & Female & Sham & Region 3 & Vessel 1 & $\mathrm{~N} / \mathrm{A}$ & 4.9 \\
\hline 6 & Female & Sham & Region 3 & Vessel 2 & N/A & 4.2 \\
\hline 6 & Female & Sham & Region 3 & Vessel 3 & N/A & 4.6 \\
\hline
\end{tabular}

PEC indicates that the muscle had a pre-existing collateral and measurements were not taken 
Cell Transplantation Vessel Count Data

\begin{tabular}{|c|c|c|c|c|c|c|c|}
\hline Replicate & Treatment & $\begin{array}{l}\text { PEC or } \\
\text { No PEC }\end{array}$ & Muscle & Region & $\begin{array}{c}\text { Total } \\
\text { Vessels } \\
\end{array}$ & Capillaries & $\begin{array}{l}\alpha \text {-SMA } \\
\text { Vessels } \\
\end{array}$ \\
\hline 1 & Myogenic Cells & PEC & Ligated & Region 1 & 15 & 7 & 8 \\
\hline 1 & Myogenic Cells & PEC & Ligated & Region 2 & 15 & 7 & 8 \\
\hline 1 & Myogenic Cells & PEC & Ligated & Region 3 & 15 & 8 & 7 \\
\hline 2 & Myogenic Cells & No PEC & Ligated & Region 1 & 13 & 7 & 6 \\
\hline 2 & Myogenic Cells & No PEC & Ligated & Region 2 & 9 & 6 & 3 \\
\hline 2 & Myogenic Cells & No PEC & Ligated & Region 3 & 14 & 9 & 5 \\
\hline 3 & Myogenic Cells & PEC & Ligated & Region 1 & 15 & 8 & 7 \\
\hline 3 & Myogenic Cells & PEC & Ligated & Region 2 & 16 & 10 & 6 \\
\hline 3 & Myogenic Cells & PEC & Ligated & Region 3 & 15 & 8 & 7 \\
\hline 4 & Myogenic Cells & No PEC & Ligated & Region 1 & 15 & 7 & 8 \\
\hline 4 & Myogenic Cells & No PEC & Ligated & Region 2 & 14 & 5 & 9 \\
\hline 4 & Myogenic Cells & No PEC & Ligated & Region 3 & 11 & 2 & 9 \\
\hline 5 & Myogenic Cells & No PEC & Ligated & Region 1 & 8 & 0 & 8 \\
\hline 5 & Myogenic Cells & No PEC & Ligated & Region 2 & 13 & 6 & 7 \\
\hline 5 & Myogenic Cells & No PEC & Ligated & Region 3 & 14 & 5 & 9 \\
\hline 6 & Myogenic Cells & No PEC & Ligated & Region 1 & 14 & 7 & 7 \\
\hline 6 & Myogenic Cells & No PEC & Ligated & Region 2 & 15 & 8 & 7 \\
\hline 6 & Myogenic Cells & No PEC & Ligated & Region 3 & 14 & 7 & 7 \\
\hline 1 & Vehicle & PEC & Ligated & Region 1 & 12 & 6 & 6 \\
\hline 1 & Vehicle & PEC & Ligated & Region 2 & 14 & 7 & 7 \\
\hline 1 & Vehicle & PEC & Ligated & Region 3 & 13 & 6 & 7 \\
\hline 2 & Vehicle & PEC & Ligated & Region 1 & 13 & 6 & 7 \\
\hline 2 & Vehicle & PEC & Ligated & Region 2 & 13 & 6 & 7 \\
\hline 2 & Vehicle & PEC & Ligated & Region 3 & 12 & 5 & 7 \\
\hline 3 & Vehicle & No PEC & Ligated & Region 1 & 11 & 5 & 6 \\
\hline 3 & Vehicle & No PEC & Ligated & Region 2 & 13 & 6 & 7 \\
\hline 3 & Vehicle & No PEC & Ligated & Region 3 & 10 & 5 & 5 \\
\hline 4 & Vehicle & No PEC & Ligated & Region 1 & 11 & 11 & \\
\hline 4 & Vehicle & No PEC & Ligated & Region 2 & 11 & 4 & 7 \\
\hline 4 & Vehicle & No PEC & Ligated & Region 3 & 15 & 8 & 7 \\
\hline 5 & Vehicle & No PEC & Ligated & Region 1 & 13 & 6 & 7 \\
\hline 5 & Vehicle & No PEC & Ligated & Region 2 & 12 & 5 & 7 \\
\hline 5 & Vehicle & No PEC & Ligated & Region 3 & 13 & 7 & 6 \\
\hline 6 & Vehicle & No PEC & Ligated & Region 1 & 13 & 6 & 7 \\
\hline 6 & Vehicle & No PEC & Ligated & Region 2 & 13 & 7 & 6 \\
\hline
\end{tabular}




\begin{tabular}{|c|c|c|c|c|c|c|c|}
\hline 6 & Vehicle & No PEC & Ligated & Region 3 & 13 & 6 & 7 \\
\hline 1 & Thrombin & No PEC & Ligated & Region 1 & 14 & 4 & 10 \\
\hline 1 & Thrombin & No PEC & Ligated & Region 2 & 14 & 5 & 9 \\
\hline 1 & Thrombin & No PEC & Ligated & Region 3 & 15 & 7 & 8 \\
\hline 2 & Thrombin & PEC & Ligated & Region 1 & 13 & 2 & 11 \\
\hline 2 & Thrombin & PEC & Ligated & Region 2 & 8 & -3 & 11 \\
\hline 2 & Thrombin & PEC & Ligated & Region 3 & 14 & 4 & 10 \\
\hline 3 & Thrombin & No PEC & Ligated & Region 1 & 14 & 4 & 10 \\
\hline 3 & Thrombin & No PEC & Ligated & Region 2 & 14 & 3 & 11 \\
\hline 3 & Thrombin & No PEC & Ligated & Region 3 & 12 & 4 & 8 \\
\hline 4 & Thrombin & No PEC & Ligated & Region 1 & 11 & 0 & 11 \\
\hline 4 & Thrombin & No PEC & Ligated & Region 2 & 14 & 2 & 12 \\
\hline 4 & Thrombin & No PEC & Ligated & Region 3 & 11 & 1 & 10 \\
\hline 5 & Thrombin & PEC & Ligated & Region 1 & 12 & 7 & 5 \\
\hline 5 & Thrombin & PEC & Ligated & Region 2 & 12 & 5 & 7 \\
\hline 5 & Thrombin & PEC & Ligated & Region 3 & 8 & 2 & 6 \\
\hline 1 & Ligation Only & PEC & Ligated & Region 1 & 13 & 6 & 7 \\
\hline 1 & Ligation Only & PEC & Ligated & Region 2 & 12 & 5 & 7 \\
\hline 1 & Ligation Only & PEC & Ligated & Region 3 & 15 & 6 & 9 \\
\hline 2 & Ligation Only & No PEC & Ligated & Region 1 & 13 & 3 & 10 \\
\hline 2 & Ligation Only & No PEC & Ligated & Region 2 & 11 & 3 & 8 \\
\hline 2 & Ligation Only & No PEC & Ligated & Region 3 & 16 & 6 & 10 \\
\hline 3 & Ligation Only & No PEC & Ligated & Region 1 & 16 & 10 & 6 \\
\hline 3 & Ligation Only & No PEC & Ligated & Region 2 & 12 & 5 & 7 \\
\hline 3 & Ligation Only & No PEC & Ligated & Region 3 & 12 & 4 & 8 \\
\hline 4 & Ligation Only & No PEC & Ligated & Region 1 & 12 & 5 & 7 \\
\hline 4 & Ligation Only & No PEC & Ligated & Region 2 & 13 & 7 & 6 \\
\hline 4 & Ligation Only & No PEC & Ligated & Region 3 & 11 & 4 & 7 \\
\hline 5 & Ligation Only & PEC & Ligated & Region 1 & 18 & 10 & 8 \\
\hline 5 & Ligation Only & PEC & Ligated & Region 2 & 16 & 10 & 6 \\
\hline 5 & Ligation Only & PEC & Ligated & Region 3 & 15 & 8 & 7 \\
\hline 1 & Myogenic Cells & PEC & Sham & Region 1 & 14 & 14 & 0 \\
\hline 1 & Myogenic Cells & PEC & Sham & Region 2 & 14 & 14 & 0 \\
\hline 1 & Myogenic Cells & PEC & Sham & Region 3 & 14 & 14 & 0 \\
\hline 2 & Myogenic Cells & No PEC & Sham & Region 1 & 10 & 10 & 0 \\
\hline 2 & Myogenic Cells & No PEC & Sham & Region 2 & 15 & 15 & 0 \\
\hline 2 & Myogenic Cells & No PEC & Sham & Region 3 & 12 & 12 & 0 \\
\hline 3 & Myogenic Cells & PEC & Sham & Region 1 & 17 & 17 & 0 \\
\hline 3 & Myogenic Cells & PEC & Sham & Region 2 & 15 & 15 & 0 \\
\hline
\end{tabular}




\begin{tabular}{|c|c|c|c|c|c|c|c|}
\hline 3 & Myogenic Cells & $\mathrm{PEC}$ & Sham & Region 3 & 13 & 13 & 0 \\
\hline 4 & Myogenic Cells & No PEC & Sham & Region 1 & 16 & 16 & 0 \\
\hline 4 & Myogenic Cells & No PEC & Sham & Region 2 & 19 & 19 & 0 \\
\hline 4 & Myogenic Cells & No PEC & Sham & Region 3 & 18 & 18 & 0 \\
\hline 5 & Myogenic Cells & No PEC & Sham & Region 1 & 16 & 16 & 0 \\
\hline 5 & Myogenic Cells & No PEC & Sham & Region 2 & 14 & 14 & 0 \\
\hline 5 & Myogenic Cells & No PEC & Sham & Region 3 & 16 & 16 & 0 \\
\hline 6 & Myogenic Cells & No PEC & Sham & Region 1 & 13 & 13 & 0 \\
\hline 6 & Myogenic Cells & No PEC & Sham & Region 2 & 13 & 13 & 0 \\
\hline 6 & Myogenic Cells & No PEC & Sham & Region 3 & 15 & 15 & 0 \\
\hline 1 & Vehicle & PEC & Sham & Region 1 & 11 & 11 & 0 \\
\hline 1 & Vehicle & PEC & Sham & Region 2 & 15 & 15 & 0 \\
\hline 1 & Vehicle & PEC & Sham & Region 3 & 17 & 17 & 0 \\
\hline 2 & Vehicle & PEC & Sham & Region 1 & 12 & 12 & 0 \\
\hline 2 & Vehicle & PEC & Sham & Region 2 & 14 & 14 & 0 \\
\hline 2 & Vehicle & PEC & Sham & Region 3 & 12 & 12 & 0 \\
\hline 3 & Vehicle & No PEC & Sham & Region 1 & 8 & 8 & 0 \\
\hline 3 & Vehicle & No PEC & Sham & Region 2 & 11 & 11 & 0 \\
\hline 3 & Vehicle & No PEC & Sham & Region 3 & 8 & 8 & 0 \\
\hline 4 & Vehicle & No PEC & Sham & Region 1 & 10 & 10 & 0 \\
\hline 4 & Vehicle & No PEC & Sham & Region 2 & 12 & 12 & 0 \\
\hline 4 & Vehicle & No PEC & Sham & Region 3 & 12 & 12 & 0 \\
\hline 5 & Vehicle & No PEC & Sham & Region 1 & 15 & 15 & 0 \\
\hline 5 & Vehicle & No PEC & Sham & Region 2 & 13 & 13 & 0 \\
\hline 5 & Vehicle & No PEC & Sham & Region 3 & 13 & 13 & 0 \\
\hline 6 & Vehicle & No PEC & Sham & Region 1 & 17 & 17 & 0 \\
\hline 6 & Vehicle & No PEC & Sham & Region 2 & 15 & 15 & 0 \\
\hline 6 & Vehicle & No PEC & Sham & Region 3 & 14 & 14 & 0 \\
\hline 1 & Thrombin & No PEC & Sham & Region 1 & 14 & 14 & 0 \\
\hline 1 & Thrombin & No PEC & Sham & Region 2 & 12 & 12 & 0 \\
\hline 1 & Thrombin & No PEC & Sham & Region 3 & 11 & 11 & 0 \\
\hline 2 & Thrombin & PEC & Sham & Region 1 & 14 & 14 & 0 \\
\hline 2 & Thrombin & PEC & Sham & Region 2 & 10 & 10 & 0 \\
\hline 2 & Thrombin & PEC & Sham & Region 3 & 10 & 10 & 0 \\
\hline 3 & Thrombin & No PEC & Sham & Region 1 & 12 & 12 & 0 \\
\hline 3 & Thrombin & No PEC & Sham & Region 2 & 14 & 14 & 0 \\
\hline 3 & Thrombin & No PEC & Sham & Region 3 & 14 & 14 & 0 \\
\hline 4 & Thrombin & No PEC & Sham & Region 1 & 12 & 12 & 0 \\
\hline 4 & Thrombin & No PEC & Sham & Region 2 & 14 & 14 & 0 \\
\hline
\end{tabular}




\begin{tabular}{|l|c|c|c|c|c|c|c|}
\hline 4 & Thrombin & No PEC & Sham & Region 3 & 16 & 16 & 0 \\
\hline 5 & Thrombin & PEC & Sham & Region 1 & 19 & 19 & 0 \\
\hline 5 & Thrombin & PEC & Sham & Region 2 & 16 & 16 & 0 \\
\hline 5 & Thrombin & PEC & Sham & Region 3 & 15 & 15 & 0 \\
\hline 1 & Ligation Only & PEC & Sham & Region 1 & 10 & 10 & 0 \\
\hline 1 & Ligation Only & PEC & Sham & Region 2 & 12 & 12 & 0 \\
\hline 1 & Ligation Only & PEC & Sham & Region 3 & 16 & 16 & 0 \\
\hline 2 & Ligation Only & No PEC & Sham & Region 1 & 12 & 12 & 0 \\
\hline 2 & Ligation Only & No PEC & Sham & Region 2 & 14 & 14 & 0 \\
\hline 2 & Ligation Only & No PEC & Sham & Region 3 & 11 & 11 & 0 \\
\hline 3 & Ligation Only & No PEC & Sham & Region 1 & 15 & 15 & 0 \\
\hline 3 & Ligation Only & No PEC & Sham & Region 2 & 16 & 16 & 0 \\
\hline 3 & Ligation Only & No PEC & Sham & Region 3 & 13 & 13 & 0 \\
\hline 4 & Ligation Only & No PEC & Sham & Region 1 & 13 & 13 & 0 \\
\hline 4 & Ligation Only & No PEC & Sham & Region 2 & 14 & 14 & 0 \\
\hline 4 & Ligation Only & No PEC & Sham & Region 3 & 18 & 18 & 0 \\
\hline 5 & Ligation Only & PEC & Sham & Region 1 & 13 & 13 & 0 \\
\hline 5 & Ligation Only & PEC & Sham & Region 2 & 14 & 14 & 0 \\
\hline 5 & Ligation Only & PEC & Sham & Region 3 & 14 & 14 & 0 \\
\hline
\end{tabular}


Cell Transplantation Vessel Diameter Data

\begin{tabular}{|c|c|c|c|c|c|c|c|}
\hline Replicate & Treatment & $\begin{array}{l}\text { PEC or } \\
\text { No PEC }\end{array}$ & Muscle & Region & Vessel & $\begin{array}{c}\alpha \text {-SMA } \\
\text { Vessel } \\
\text { Diameter } \\
(\mu \mathrm{m}) \\
\end{array}$ & $\begin{array}{c}\text { Lectin } \\
\text { Vessel } \\
\text { Diameter } \\
(\mu \mathrm{m}) \\
\end{array}$ \\
\hline 1 & Myogenic Cells & PEC & Ligated & Region 1 & Vessel 1 & 9.0 & 5.0 \\
\hline 1 & Myogenic Cells & PEC & Ligated & Region 1 & Vessel 2 & 8.6 & 4.2 \\
\hline 1 & Myogenic Cells & PEC & Ligated & Region 1 & Vessel 3 & 7.7 & 5.7 \\
\hline 1 & Myogenic Cells & PEC & Ligated & Region 2 & Vessel 1 & 10.6 & 4.1 \\
\hline 1 & Myogenic Cells & PEC & Ligated & Region 2 & Vessel 2 & 8.0 & 3.5 \\
\hline 1 & Myogenic Cells & PEC & Ligated & Region 2 & Vessel 3 & 12.3 & 5.1 \\
\hline 1 & Myogenic Cells & PEC & Ligated & Region 3 & Vessel 1 & 7.9 & 6.2 \\
\hline 1 & Myogenic Cells & PEC & Ligated & Region 3 & Vessel 2 & 9.2 & 4.4 \\
\hline 1 & Myogenic Cells & PEC & Ligated & Region 3 & Vessel 3 & 8.1 & 3.7 \\
\hline 2 & Myogenic Cells & No PEC & Ligated & Region 1 & Vessel 1 & 16.0 & 5.7 \\
\hline 2 & Myogenic Cells & No PEC & Ligated & Region 1 & Vessel 2 & 18.9 & 5.3 \\
\hline 2 & Myogenic Cells & No PEC & Ligated & Region 1 & Vessel 3 & 18.7 & 5.4 \\
\hline 2 & Myogenic Cells & No PEC & Ligated & Region 2 & Vessel 1 & 28.2 & 4.9 \\
\hline 2 & Myogenic Cells & No PEC & Ligated & Region 2 & Vessel 2 & 26.3 & 5.3 \\
\hline 2 & Myogenic Cells & No PEC & Ligated & Region 2 & Vessel 3 & 43.4 & 4.9 \\
\hline 2 & Myogenic Cells & No PEC & Ligated & Region 3 & Vessel 1 & 10.7 & 8.0 \\
\hline 2 & Myogenic Cells & No PEC & Ligated & Region 3 & Vessel 2 & 15.8 & 6.9 \\
\hline 2 & Myogenic Cells & No PEC & Ligated & Region 3 & Vessel 3 & 12.9 & 7.4 \\
\hline 3 & Myogenic Cells & PEC & Ligated & Region 1 & Vessel 1 & 10.1 & 6.6 \\
\hline 3 & Myogenic Cells & PEC & Ligated & Region 1 & Vessel 2 & 10.3 & 5.8 \\
\hline 3 & Myogenic Cells & PEC & Ligated & Region 1 & Vessel 3 & 7.7 & 6.0 \\
\hline 3 & Myogenic Cells & PEC & Ligated & Region 2 & Vessel 1 & 12.4 & 8.7 \\
\hline 3 & Myogenic Cells & PEC & Ligated & Region 2 & Vessel 2 & 9.9 & 7.5 \\
\hline 3 & Myogenic Cells & PEC & Ligated & Region 2 & Vessel 3 & 11.9 & 8.6 \\
\hline 3 & Myogenic Cells & PEC & Ligated & Region 3 & Vessel 1 & 14.0 & 6.6 \\
\hline 3 & Myogenic Cells & PEC & Ligated & Region 3 & Vessel 2 & 13.7 & 4.9 \\
\hline 3 & Myogenic Cells & PEC & Ligated & Region 3 & Vessel 3 & 10.4 & 6.4 \\
\hline 4 & Myogenic Cells & No PEC & Ligated & Region 1 & Vessel 1 & 11.5 & 4.7 \\
\hline 4 & Myogenic Cells & No PEC & Ligated & Region 1 & Vessel 2 & 8.4 & 4.1 \\
\hline 4 & Myogenic Cells & No PEC & Ligated & Region 1 & Vessel 3 & 8.9 & 4.1 \\
\hline 4 & Myogenic Cells & No PEC & Ligated & Region 2 & Vessel 1 & 9.0 & 4.8 \\
\hline 4 & Myogenic Cells & No PEC & Ligated & Region 2 & Vessel 2 & 8.1 & 5.3 \\
\hline 4 & Myogenic Cells & No PEC & Ligated & Region 2 & Vessel 3 & 9.1 & 5.0 \\
\hline 4 & Myogenic Cells & No PEC & Ligated & Region 3 & Vessel 1 & 10.5 & 4.4 \\
\hline
\end{tabular}




\begin{tabular}{|c|c|c|c|c|c|c|c|}
\hline 4 & Myogenic Cells & No PEC & Ligated & Region 3 & Vessel 2 & 8.3 & 3.9 \\
\hline 4 & Myogenic Cells & No PEC & Ligated & Region 3 & Vessel 3 & 10.7 & 3.6 \\
\hline 5 & Myogenic Cells & No PEC & Ligated & Region 1 & Vessel 1 & 12.2 & 4.8 \\
\hline 5 & Myogenic Cells & No PEC & Ligated & Region 1 & Vessel 2 & 14.5 & 5.6 \\
\hline 5 & Myogenic Cells & No PEC & Ligated & Region 1 & Vessel 3 & 13.9 & 4.7 \\
\hline 5 & Myogenic Cells & No PEC & Ligated & Region 2 & Vessel 1 & 19.9 & 4.7 \\
\hline 5 & Myogenic Cells & No PEC & Ligated & Region 2 & Vessel 2 & 20.5 & 4.7 \\
\hline 5 & Myogenic Cells & No PEC & Ligated & Region 2 & Vessel 3 & 20.7 & 5.1 \\
\hline 5 & Myogenic Cells & No PEC & Ligated & Region 3 & Vessel 1 & 10.4 & 3.6 \\
\hline 5 & Myogenic Cells & No PEC & Ligated & Region 3 & Vessel 2 & 10.9 & 4.0 \\
\hline 5 & Myogenic Cells & No PEC & Ligated & Region 3 & Vessel 3 & 10.4 & 3.9 \\
\hline 6 & Myogenic Cells & No PEC & Ligated & Region 1 & Vessel 1 & 18.9 & 4.9 \\
\hline 6 & Myogenic Cells & No PEC & Ligated & Region 1 & Vessel 2 & 16.2 & 4.1 \\
\hline 6 & Myogenic Cells & No PEC & Ligated & Region 1 & Vessel 3 & 17.9 & 5.8 \\
\hline 6 & Myogenic Cells & No PEC & Ligated & Region 2 & Vessel 1 & 11.3 & 5.2 \\
\hline 6 & Myogenic Cells & No PEC & Ligated & Region 2 & Vessel 2 & 14.6 & 5.1 \\
\hline 6 & Myogenic Cells & No PEC & Ligated & Region 2 & Vessel 3 & 11.0 & 4.5 \\
\hline 6 & Myogenic Cells & No PEC & Ligated & Region 3 & Vessel 1 & 18.0 & 5.0 \\
\hline 6 & Myogenic Cells & No PEC & Ligated & Region 3 & Vessel 2 & 15.2 & 5.4 \\
\hline 6 & Myogenic Cells & No PEC & Ligated & Region 3 & Vessel 3 & 15.4 & 5.5 \\
\hline 1 & Vehicle & PEC & Ligated & Region 1 & Vessel 1 & 15.6 & 6.1 \\
\hline 1 & Vehicle & PEC & Ligated & Region 1 & Vessel 2 & 13.3 & 5.9 \\
\hline 1 & Vehicle & PEC & Ligated & Region 1 & Vessel 3 & 14.8 & 5.2 \\
\hline 1 & Vehicle & PEC & Ligated & Region 2 & Vessel 1 & 14.9 & 4.7 \\
\hline 1 & Vehicle & PEC & Ligated & Region 2 & Vessel 2 & 12.5 & 4.6 \\
\hline 1 & Vehicle & PEC & Ligated & Region 2 & Vessel 3 & 7.6 & 4.4 \\
\hline 1 & Vehicle & PEC & Ligated & Region 3 & Vessel 1 & 7.3 & 5.0 \\
\hline 1 & Vehicle & PEC & Ligated & Region 3 & Vessel 2 & 7.6 & 5.0 \\
\hline 1 & Vehicle & PEC & Ligated & Region 3 & Vessel 3 & 6.9 & 7.3 \\
\hline 2 & Vehicle & PEC & Ligated & Region 1 & Vessel 1 & 6.8 & 4.6 \\
\hline 2 & Vehicle & PEC & Ligated & Region 1 & Vessel 2 & 6.3 & 3.5 \\
\hline 2 & Vehicle & PEC & Ligated & Region 1 & Vessel 3 & 4.5 & 5.1 \\
\hline 2 & Vehicle & PEC & Ligated & Region 2 & Vessel 1 & 9.6 & 5.0 \\
\hline 2 & Vehicle & PEC & Ligated & Region 2 & Vessel 2 & 9.2 & 4.6 \\
\hline 2 & Vehicle & PEC & Ligated & Region 2 & Vessel 3 & 8.8 & 4.7 \\
\hline 2 & Vehicle & PEC & Ligated & Region 3 & Vessel 1 & 9.5 & 3.9 \\
\hline 2 & Vehicle & PEC & Ligated & Region 3 & Vessel 2 & 5.9 & 4.8 \\
\hline 2 & Vehicle & PEC & Ligated & Region 3 & Vessel 3 & 8.4 & 5.5 \\
\hline 3 & Vehicle & No PEC & Ligated & Region 1 & Vessel 1 & 11.7 & 7.4 \\
\hline
\end{tabular}




\begin{tabular}{|c|c|c|c|c|c|c|c|}
\hline 3 & Vehicle & No PEC & Ligated & Region 1 & Vessel 2 & 7.8 & 5.7 \\
\hline 3 & Vehicle & No PEC & Ligated & Region 1 & Vessel 3 & 7.3 & 6.8 \\
\hline 3 & Vehicle & No PEC & Ligated & Region 2 & Vessel 1 & 11.5 & 4.6 \\
\hline 3 & Vehicle & No PEC & Ligated & Region 2 & Vessel 2 & 9.6 & 5.9 \\
\hline 3 & Vehicle & No PEC & Ligated & Region 2 & Vessel 3 & 7.6 & 3.5 \\
\hline 3 & Vehicle & No PEC & Ligated & Region 3 & Vessel 1 & 8.4 & 6.0 \\
\hline 3 & Vehicle & No PEC & Ligated & Region 3 & Vessel 2 & 8.0 & 6.3 \\
\hline 3 & Vehicle & No PEC & Ligated & Region 3 & Vessel 3 & 7.7 & 7.6 \\
\hline 4 & Vehicle & No PEC & Ligated & Region 1 & Vessel 1 & & 5.5 \\
\hline 4 & Vehicle & No PEC & Ligated & Region 1 & Vessel 2 & & 5.1 \\
\hline 4 & Vehicle & No PEC & Ligated & Region 1 & Vessel 3 & & 4.4 \\
\hline 4 & Vehicle & No PEC & Ligated & Region 2 & Vessel 1 & 9.4 & 5.1 \\
\hline 4 & Vehicle & No PEC & Ligated & Region 2 & Vessel 2 & 16.9 & 4.8 \\
\hline 4 & Vehicle & No PEC & Ligated & Region 2 & Vessel 3 & 8.0 & 4.9 \\
\hline 4 & Vehicle & No PEC & Ligated & Region 3 & Vessel 1 & 11.3 & 5.9 \\
\hline 4 & Vehicle & No PEC & Ligated & Region 3 & Vessel 2 & 8.9 & 6.1 \\
\hline 4 & Vehicle & No PEC & Ligated & Region 3 & Vessel 3 & 11.6 & 5.7 \\
\hline 5 & Vehicle & No PEC & Ligated & Region 1 & Vessel 1 & 11.0 & 7.8 \\
\hline 5 & Vehicle & No PEC & Ligated & Region 1 & Vessel 2 & 8.2 & 4.1 \\
\hline 5 & Vehicle & No PEC & Ligated & Region 1 & Vessel 3 & 9.5 & 5.2 \\
\hline 5 & Vehicle & No PEC & Ligated & Region 2 & Vessel 1 & 8.3 & 4.3 \\
\hline 5 & Vehicle & No PEC & Ligated & Region 2 & Vessel 2 & 9.5 & 5.0 \\
\hline 5 & Vehicle & No PEC & Ligated & Region 2 & Vessel 3 & 9.6 & 7.5 \\
\hline 5 & Vehicle & No PEC & Ligated & Region 3 & Vessel 1 & 12.6 & 4.3 \\
\hline 5 & Vehicle & No PEC & Ligated & Region 3 & Vessel 2 & 14.3 & 4.8 \\
\hline 5 & Vehicle & No PEC & Ligated & Region 3 & Vessel 3 & 12.1 & 5.1 \\
\hline 6 & Vehicle & No PEC & Ligated & Region 1 & Vessel 1 & 14.0 & 4.3 \\
\hline 6 & Vehicle & No PEC & Ligated & Region 1 & Vessel 2 & 8.7 & 4.0 \\
\hline 6 & Vehicle & No PEC & Ligated & Region 1 & Vessel 3 & 11.5 & 4.1 \\
\hline 6 & Vehicle & No PEC & Ligated & Region 2 & Vessel 1 & 9.0 & 3.4 \\
\hline 6 & Vehicle & No PEC & Ligated & Region 2 & Vessel 2 & 14.9 & 4.1 \\
\hline 6 & Vehicle & No PEC & Ligated & Region 2 & Vessel 3 & 13.2 & 3.2 \\
\hline 6 & Vehicle & No PEC & Ligated & Region 3 & Vessel 1 & 10.0 & 3.7 \\
\hline 6 & Vehicle & No PEC & Ligated & Region 3 & Vessel 2 & 10.6 & 4.1 \\
\hline 6 & Vehicle & No PEC & Ligated & Region 3 & Vessel 3 & 12.5 & 5.0 \\
\hline 1 & Thrombin & No PEC & Ligated & Region 1 & Vessel 1 & 11.2 & 4.7 \\
\hline 1 & Thrombin & No PEC & Ligated & Region 1 & Vessel 2 & 13.8 & 4.5 \\
\hline 1 & Thrombin & No PEC & Ligated & Region 1 & Vessel 3 & 15.0 & 5.6 \\
\hline 1 & Thrombin & No PEC & Ligated & Region 2 & Vessel 1 & 22.3 & 3.5 \\
\hline
\end{tabular}




\begin{tabular}{|c|c|c|c|c|c|c|c|}
\hline 1 & Thrombin & No PEC & Ligated & Region 2 & Vessel 2 & 15.8 & 3.3 \\
\hline 1 & Thrombin & No PEC & Ligated & Region 2 & Vessel 3 & 11.2 & 4.5 \\
\hline 1 & Thrombin & No PEC & Ligated & Region 3 & Vessel 1 & 12.1 & 5.0 \\
\hline 1 & Thrombin & No PEC & Ligated & Region 3 & Vessel 2 & 12.9 & 4.3 \\
\hline 1 & Thrombin & No PEC & Ligated & Region 3 & Vessel 3 & 15.4 & 4.5 \\
\hline 2 & Thrombin & PEC & Ligated & Region 1 & Vessel 1 & 11.3 & 4.1 \\
\hline 2 & Thrombin & PEC & Ligated & Region 1 & Vessel 2 & 13.8 & 4.4 \\
\hline 2 & Thrombin & PEC & Ligated & Region 1 & Vessel 3 & 9.7 & 4.2 \\
\hline 2 & Thrombin & PEC & Ligated & Region 2 & Vessel 1 & 16.2 & 5.9 \\
\hline 2 & Thrombin & PEC & Ligated & Region 2 & Vessel 2 & 11.5 & 4.8 \\
\hline 2 & Thrombin & PEC & Ligated & Region 2 & Vessel 3 & 13.3 & 4.9 \\
\hline 2 & Thrombin & PEC & Ligated & Region 3 & Vessel 1 & 9.0 & 4.0 \\
\hline 2 & Thrombin & PEC & Ligated & Region 3 & Vessel 2 & 11.7 & 4.1 \\
\hline 2 & Thrombin & PEC & Ligated & Region 3 & Vessel 3 & 8.6 & 4.6 \\
\hline 3 & Thrombin & No PEC & Ligated & Region 1 & Vessel 1 & 15.7 & 4.8 \\
\hline 3 & Thrombin & No PEC & Ligated & Region 1 & Vessel 2 & 14.4 & 5.1 \\
\hline 3 & Thrombin & No PEC & Ligated & Region 1 & Vessel 3 & 16.7 & 5.0 \\
\hline 3 & Thrombin & No PEC & Ligated & Region 2 & Vessel 1 & 10.3 & 4.7 \\
\hline 3 & Thrombin & No PEC & Ligated & Region 2 & Vessel 2 & 10.1 & 5.2 \\
\hline 3 & Thrombin & No PEC & Ligated & Region 2 & Vessel 3 & 11.0 & 5.2 \\
\hline 3 & Thrombin & No PEC & Ligated & Region 3 & Vessel 1 & 11.5 & 4.5 \\
\hline 3 & Thrombin & No PEC & Ligated & Region 3 & Vessel 2 & 9.2 & 4.9 \\
\hline 3 & Thrombin & No PEC & Ligated & Region 3 & Vessel 3 & 7.0 & 4.9 \\
\hline 4 & Thrombin & No PEC & Ligated & Region 1 & Vessel 1 & 14.2 & 5.2 \\
\hline 4 & Thrombin & No PEC & Ligated & Region 1 & Vessel 2 & 11.8 & 5.3 \\
\hline 4 & Thrombin & No PEC & Ligated & Region 1 & Vessel 3 & 14.1 & 3.7 \\
\hline 4 & Thrombin & No PEC & Ligated & Region 2 & Vessel 1 & 14.9 & 3.8 \\
\hline 4 & Thrombin & No PEC & Ligated & Region 2 & Vessel 2 & 11.9 & 5.6 \\
\hline 4 & Thrombin & No PEC & Ligated & Region 2 & Vessel 3 & 11.8 & 4.9 \\
\hline 4 & Thrombin & No PEC & Ligated & Region 3 & Vessel 1 & 15.0 & 4.6 \\
\hline 4 & Thrombin & No PEC & Ligated & Region 3 & Vessel 2 & 14.1 & 5.5 \\
\hline 4 & Thrombin & No PEC & Ligated & Region 3 & Vessel 3 & 19.2 & 4.1 \\
\hline 5 & Thrombin & PEC & Ligated & Region 1 & Vessel 1 & 7.5 & 4.5 \\
\hline 5 & Thrombin & PEC & Ligated & Region 1 & Vessel 2 & 5.0 & 5.5 \\
\hline 5 & Thrombin & PEC & Ligated & Region 1 & Vessel 3 & 6.5 & 3.0 \\
\hline 5 & Thrombin & PEC & Ligated & Region 2 & Vessel 1 & 8.8 & 5.1 \\
\hline 5 & Thrombin & PEC & Ligated & Region 2 & Vessel 2 & 7.3 & 2.6 \\
\hline 5 & Thrombin & PEC & Ligated & Region 2 & Vessel 3 & 6.8 & 4.8 \\
\hline 5 & Thrombin & PEC & Ligated & Region 3 & Vessel 1 & 7.3 & 4.5 \\
\hline
\end{tabular}




\begin{tabular}{|c|c|c|c|c|c|c|c|}
\hline 5 & Thrombin & PEC & Ligated & Region 3 & Vessel 2 & 8.5 & 5.5 \\
\hline 5 & Thrombin & PEC & Ligated & Region 3 & Vessel 3 & 10.5 & 4.7 \\
\hline 1 & Ligation Only & PEC & Ligated & Region 1 & Vessel 1 & 13.2 & 4.0 \\
\hline 1 & Ligation Only & PEC & Ligated & Region 1 & Vessel 2 & 14.6 & 4.3 \\
\hline 1 & Ligation Only & PEC & Ligated & Region 1 & Vessel 3 & 4.6 & 3.8 \\
\hline 1 & Ligation Only & PEC & Ligated & Region 2 & Vessel 1 & 7.7 & 3.3 \\
\hline 1 & Ligation Only & PEC & Ligated & Region 2 & Vessel 2 & 6.9 & 4.6 \\
\hline 1 & Ligation Only & PEC & Ligated & Region 2 & Vessel 3 & 9.1 & 4.0 \\
\hline 1 & Ligation Only & PEC & Ligated & Region 3 & Vessel 1 & 6.4 & 5.9 \\
\hline 1 & Ligation Only & PEC & Ligated & Region 3 & Vessel 2 & 7.0 & 4.1 \\
\hline 1 & Ligation Only & PEC & Ligated & Region 3 & Vessel 3 & 4.3 & 3.2 \\
\hline 2 & Ligation Only & No PEC & Ligated & Region 1 & Vessel 1 & 12.1 & 4.9 \\
\hline 2 & Ligation Only & No PEC & Ligated & Region 1 & Vessel 2 & 13.5 & 5.9 \\
\hline 2 & Ligation Only & No PEC & Ligated & Region 1 & Vessel 3 & 14.6 & 4.3 \\
\hline 2 & Ligation Only & No PEC & Ligated & Region 2 & Vessel 1 & 13.4 & 6.2 \\
\hline 2 & Ligation Only & No PEC & Ligated & Region 2 & Vessel 2 & 12.3 & 5.6 \\
\hline 2 & Ligation Only & No PEC & Ligated & Region 2 & Vessel 3 & 11.5 & 3.7 \\
\hline 2 & Ligation Only & No PEC & Ligated & Region 3 & Vessel 1 & 9.0 & 3.5 \\
\hline 2 & Ligation Only & No PEC & Ligated & Region 3 & Vessel 2 & 9.3 & 4.4 \\
\hline 2 & Ligation Only & No PEC & Ligated & Region 3 & Vessel 3 & 6.4 & 4.9 \\
\hline 3 & Ligation Only & No PEC & Ligated & Region 1 & Vessel 1 & 15.2 & 5.1 \\
\hline 3 & Ligation Only & No PEC & Ligated & Region 1 & Vessel 2 & 10.9 & 5.2 \\
\hline 3 & Ligation Only & No PEC & Ligated & Region 1 & Vessel 3 & 7.8 & 5.2 \\
\hline 3 & Ligation Only & No PEC & Ligated & Region 2 & Vessel 1 & 13.0 & 6.0 \\
\hline 3 & Ligation Only & No PEC & Ligated & Region 2 & Vessel 2 & 15.2 & 6.1 \\
\hline 3 & Ligation Only & No PEC & Ligated & Region 2 & Vessel 3 & 10.1 & 4.8 \\
\hline 3 & Ligation Only & No PEC & Ligated & Region 3 & Vessel 1 & 12.1 & 7.0 \\
\hline 3 & Ligation Only & No PEC & Ligated & Region 3 & Vessel 2 & 10.6 & 7.0 \\
\hline 3 & Ligation Only & No PEC & Ligated & Region 3 & Vessel 3 & 10.5 & 5.8 \\
\hline 4 & Ligation Only & No PEC & Ligated & Region 1 & Vessel 1 & 14.7 & 5.4 \\
\hline 4 & Ligation Only & No PEC & Ligated & Region 1 & Vessel 2 & 11.0 & 4.8 \\
\hline 4 & Ligation Only & No PEC & Ligated & Region 1 & Vessel 3 & 10.6 & 5.6 \\
\hline 4 & Ligation Only & No PEC & Ligated & Region 2 & Vessel 1 & 9.6 & 6.1 \\
\hline 4 & Ligation Only & No PEC & Ligated & Region 2 & Vessel 2 & 13.3 & 6.3 \\
\hline 4 & Ligation Only & No PEC & Ligated & Region 2 & Vessel 3 & 14.2 & 5.8 \\
\hline 4 & Ligation Only & No PEC & Ligated & Region 3 & Vessel 1 & 17.4 & 3.8 \\
\hline 4 & Ligation Only & No PEC & Ligated & Region 3 & Vessel 2 & 15.5 & 5.9 \\
\hline 4 & Ligation Only & No PEC & Ligated & Region 3 & Vessel 3 & 10.3 & 4.8 \\
\hline 5 & Ligation Only & PEC & Ligated & Region 1 & Vessel 1 & 6.4 & 5.1 \\
\hline
\end{tabular}




\begin{tabular}{|c|c|c|c|c|c|c|c|}
\hline 5 & Ligation Only & PEC & Ligated & Region 1 & Vessel 2 & 9.6 & 5.4 \\
\hline 5 & Ligation Only & PEC & Ligated & Region 1 & Vessel 3 & 5.2 & 5.2 \\
\hline 5 & Ligation Only & PEC & Ligated & Region 2 & Vessel 1 & 10.6 & 4.5 \\
\hline 5 & Ligation Only & PEC & Ligated & Region 2 & Vessel 2 & 8.1 & 5.0 \\
\hline 5 & Ligation Only & PEC & Ligated & Region 2 & Vessel 3 & 7.7 & 4.2 \\
\hline 5 & Ligation Only & PEC & Ligated & Region 3 & Vessel 1 & 6.7 & 5.0 \\
\hline 5 & Ligation Only & PEC & Ligated & Region 3 & Vessel 2 & 9.0 & 4.9 \\
\hline 5 & Ligation Only & PEC & Ligated & Region 3 & Vessel 3 & 8.9 & 6.2 \\
\hline 1 & Myogenic Cells & PEC & Sham & Region 1 & Vessel 1 & $\mathrm{~N} / \mathrm{A}$ & 4.3 \\
\hline 1 & Myogenic Cells & PEC & Sham & Region 1 & Vessel 2 & N/A & 4.5 \\
\hline 1 & Myogenic Cells & PEC & Sham & Region 1 & Vessel 3 & N/A & 4.8 \\
\hline 1 & Myogenic Cells & PEC & Sham & Region 2 & Vessel 1 & N/A & 7.1 \\
\hline 1 & Myogenic Cells & PEC & Sham & Region 2 & Vessel 2 & N/A & 4.8 \\
\hline 1 & Myogenic Cells & PEC & Sham & Region 2 & Vessel 3 & N/A & 7.2 \\
\hline 1 & Myogenic Cells & PEC & Sham & Region 3 & Vessel 1 & $\mathrm{~N} / \mathrm{A}$ & 4.5 \\
\hline 1 & Myogenic Cells & PEC & Sham & Region 3 & Vessel 2 & N/A & 5.5 \\
\hline 1 & Myogenic Cells & PEC & Sham & Region 3 & Vessel 3 & N/A & 5.2 \\
\hline 2 & Myogenic Cells & No PEC & Sham & Region 1 & Vessel 1 & $\mathrm{~N} / \mathrm{A}$ & 6.0 \\
\hline 2 & Myogenic Cells & No PEC & Sham & Region 1 & Vessel 2 & N/A & 4.6 \\
\hline 2 & Myogenic Cells & No PEC & Sham & Region 1 & Vessel 3 & $\mathrm{~N} / \mathrm{A}$ & 5.7 \\
\hline 2 & Myogenic Cells & No PEC & Sham & Region 2 & Vessel 1 & N/A & 5.1 \\
\hline 2 & Myogenic Cells & No PEC & Sham & Region 2 & Vessel 2 & $\mathrm{~N} / \mathrm{A}$ & 4.0 \\
\hline 2 & Myogenic Cells & No PEC & Sham & Region 2 & Vessel 3 & N/A & 4.1 \\
\hline 2 & Myogenic Cells & No PEC & Sham & Region 3 & Vessel 1 & N/A & 4.2 \\
\hline 2 & Myogenic Cells & No PEC & Sham & Region 3 & Vessel 2 & N/A & 4.3 \\
\hline 2 & Myogenic Cells & No PEC & Sham & Region 3 & Vessel 3 & N/A & 5.0 \\
\hline 3 & Myogenic Cells & PEC & Sham & Region 1 & Vessel 1 & $\mathrm{~N} / \mathrm{A}$ & 5.0 \\
\hline 3 & Myogenic Cells & PEC & Sham & Region 1 & Vessel 2 & N/A & 4.6 \\
\hline 3 & Myogenic Cells & PEC & Sham & Region 1 & Vessel 3 & N/A & 4.4 \\
\hline 3 & Myogenic Cells & PEC & Sham & Region 2 & Vessel 1 & N/A & 4.7 \\
\hline 3 & Myogenic Cells & PEC & Sham & Region 2 & Vessel 2 & $\mathrm{~N} / \mathrm{A}$ & 4.6 \\
\hline 3 & Myogenic Cells & PEC & Sham & Region 2 & Vessel 3 & N/A & 4.0 \\
\hline 3 & Myogenic Cells & PEC & Sham & Region 3 & Vessel 1 & $\mathrm{~N} / \mathrm{A}$ & 4.7 \\
\hline 3 & Myogenic Cells & PEC & Sham & Region 3 & Vessel 2 & N/A & 4.4 \\
\hline 3 & Myogenic Cells & PEC & Sham & Region 3 & Vessel 3 & N/A & 3.8 \\
\hline 4 & Myogenic Cells & No PEC & Sham & Region 1 & Vessel 1 & N/A & 4.2 \\
\hline 4 & Myogenic Cells & No PEC & Sham & Region 1 & Vessel 2 & $\mathrm{~N} / \mathrm{A}$ & 4.8 \\
\hline 4 & Myogenic Cells & No PEC & Sham & Region 1 & Vessel 3 & $\mathrm{~N} / \mathrm{A}$ & 4.1 \\
\hline 4 & Myogenic Cells & No PEC & Sham & Region 2 & Vessel 1 & $\mathrm{~N} / \mathrm{A}$ & 4.9 \\
\hline
\end{tabular}




\begin{tabular}{|c|c|c|c|c|c|c|c|}
\hline 4 & Myogenic Cells & No PEC & Sham & Region 2 & Vessel 2 & N/A & 5.7 \\
\hline 4 & Myogenic Cells & No PEC & Sham & Region 2 & Vessel 3 & N/A & 4.4 \\
\hline 4 & Myogenic Cells & No PEC & Sham & Region 3 & Vessel 1 & N/A & 5.9 \\
\hline 4 & Myogenic Cells & No PEC & Sham & Region 3 & Vessel 2 & N/A & 5.5 \\
\hline 4 & Myogenic Cells & No PEC & Sham & Region 3 & Vessel 3 & N/A & 5.3 \\
\hline 5 & Myogenic Cells & No PEC & Sham & Region 1 & Vessel 1 & N/A & 4.4 \\
\hline 5 & Myogenic Cells & No PEC & Sham & Region 1 & Vessel 2 & N/A & 4.2 \\
\hline 5 & Myogenic Cells & No PEC & Sham & Region 1 & Vessel 3 & N/A & 3.9 \\
\hline 5 & Myogenic Cells & No PEC & Sham & Region 2 & Vessel 1 & $\mathrm{~N} / \mathrm{A}$ & 5.8 \\
\hline 5 & Myogenic Cells & No PEC & Sham & Region 2 & Vessel 2 & N/A & 5.9 \\
\hline 5 & Myogenic Cells & No PEC & Sham & Region 2 & Vessel 3 & N/A & 5.4 \\
\hline 5 & Myogenic Cells & No PEC & Sham & Region 3 & Vessel 1 & N/A & 6.4 \\
\hline 5 & Myogenic Cells & No PEC & Sham & Region 3 & Vessel 2 & N/A & 4.7 \\
\hline 5 & Myogenic Cells & No PEC & Sham & Region 3 & Vessel 3 & N/A & 5.0 \\
\hline 6 & Myogenic Cells & No PEC & Sham & Region 1 & Vessel 1 & N/A & 5.6 \\
\hline 6 & Myogenic Cells & No PEC & Sham & Region 1 & Vessel 2 & N/A & 5.1 \\
\hline 6 & Myogenic Cells & No PEC & Sham & Region 1 & Vessel 3 & N/A & 4.7 \\
\hline 6 & Myogenic Cells & No PEC & Sham & Region 2 & Vessel 1 & $\mathrm{~N} / \mathrm{A}$ & 5.6 \\
\hline 6 & Myogenic Cells & No PEC & Sham & Region 2 & Vessel 2 & N/A & 5.3 \\
\hline 6 & Myogenic Cells & No PEC & Sham & Region 2 & Vessel 3 & $\mathrm{~N} / \mathrm{A}$ & 6.0 \\
\hline 6 & Myogenic Cells & No PEC & Sham & Region 3 & Vessel 1 & N/A & 5.5 \\
\hline 6 & Myogenic Cells & No PEC & Sham & Region 3 & Vessel 2 & $\mathrm{~N} / \mathrm{A}$ & 4.7 \\
\hline 6 & Myogenic Cells & No PEC & Sham & Region 3 & Vessel 3 & N/A & 4.4 \\
\hline 1 & Vehicle & PEC & Sham & Region 1 & Vessel 1 & N/A & 4.2 \\
\hline 1 & Vehicle & PEC & Sham & Region 1 & Vessel 2 & N/A & 3.6 \\
\hline 1 & Vehicle & PEC & Sham & Region 1 & Vessel 3 & N/A & 3.9 \\
\hline 1 & Vehicle & PEC & Sham & Region 2 & Vessel 1 & N/A & 4.6 \\
\hline 1 & Vehicle & PEC & Sham & Region 2 & Vessel 2 & N/A & 5.3 \\
\hline 1 & Vehicle & PEC & Sham & Region 2 & Vessel 3 & N/A & 5.8 \\
\hline 1 & Vehicle & PEC & Sham & Region 3 & Vessel 1 & N/A & 5.5 \\
\hline 1 & Vehicle & PEC & Sham & Region 3 & Vessel 2 & N/A & 5.0 \\
\hline 1 & Vehicle & PEC & Sham & Region 3 & Vessel 3 & N/A & 5.0 \\
\hline 2 & Vehicle & PEC & Sham & Region 1 & Vessel 1 & N/A & 5.6 \\
\hline 2 & Vehicle & PEC & Sham & Region 1 & Vessel 2 & N/A & 5.0 \\
\hline 2 & Vehicle & PEC & Sham & Region 1 & Vessel 3 & N/A & 4.3 \\
\hline 2 & Vehicle & PEC & Sham & Region 2 & Vessel 1 & N/A & 4.1 \\
\hline 2 & Vehicle & PEC & Sham & Region 2 & Vessel 2 & N/A & 4.3 \\
\hline 2 & Vehicle & PEC & Sham & Region 2 & Vessel 3 & N/A & 4.4 \\
\hline 2 & Vehicle & PEC & Sham & Region 3 & Vessel 1 & N/A & 4.0 \\
\hline
\end{tabular}




\begin{tabular}{|c|c|c|c|c|c|c|c|}
\hline 2 & Vehicle & PEC & Sham & Region 3 & Vessel 2 & N/A & 4.6 \\
\hline 2 & Vehicle & PEC & Sham & Region 3 & Vessel 3 & N/A & 4.9 \\
\hline 3 & Vehicle & No PEC & Sham & Region 1 & Vessel 1 & N/A & 4.1 \\
\hline 3 & Vehicle & No PEC & Sham & Region 1 & Vessel 2 & N/A & 5.0 \\
\hline 3 & Vehicle & No PEC & Sham & Region 1 & Vessel 3 & $\mathrm{~N} / \mathrm{A}$ & 3.9 \\
\hline 3 & Vehicle & No PEC & Sham & Region 2 & Vessel 1 & N/A & 6.1 \\
\hline 3 & Vehicle & No PEC & Sham & Region 2 & Vessel 2 & N/A & 6.3 \\
\hline 3 & Vehicle & No PEC & Sham & Region 2 & Vessel 3 & N/A & 4.6 \\
\hline 3 & Vehicle & No PEC & Sham & Region 3 & Vessel 1 & N/A & 3.5 \\
\hline 3 & Vehicle & No PEC & Sham & Region 3 & Vessel 2 & N/A & 6.4 \\
\hline 3 & Vehicle & No PEC & Sham & Region 3 & Vessel 3 & N/A & 5.9 \\
\hline 4 & Vehicle & No PEC & Sham & Region 1 & Vessel 1 & N/A & 5.4 \\
\hline 4 & Vehicle & No PEC & Sham & Region 1 & Vessel 2 & N/A & 5.6 \\
\hline 4 & Vehicle & No PEC & Sham & Region 1 & Vessel 3 & N/A & 5.1 \\
\hline 4 & Vehicle & No PEC & Sham & Region 2 & Vessel 1 & N/A & 5.4 \\
\hline 4 & Vehicle & No PEC & Sham & Region 2 & Vessel 2 & N/A & 7.5 \\
\hline 4 & Vehicle & No PEC & Sham & Region 2 & Vessel 3 & N/A & 5.6 \\
\hline 4 & Vehicle & No PEC & Sham & Region 3 & Vessel 1 & N/A & 4.8 \\
\hline 4 & Vehicle & No PEC & Sham & Region 3 & Vessel 2 & N/A & 6.2 \\
\hline 4 & Vehicle & No PEC & Sham & Region 3 & Vessel 3 & N/A & 4.8 \\
\hline 5 & Vehicle & No PEC & Sham & Region 1 & Vessel 1 & N/A & 4.8 \\
\hline 5 & Vehicle & No PEC & Sham & Region 1 & Vessel 2 & N/A & 6.3 \\
\hline 5 & Vehicle & No PEC & Sham & Region 1 & Vessel 3 & N/A & 4.9 \\
\hline 5 & Vehicle & No PEC & Sham & Region 2 & Vessel 1 & N/A & 6.1 \\
\hline 5 & Vehicle & No PEC & Sham & Region 2 & Vessel 2 & N/A & 5.5 \\
\hline 5 & Vehicle & No PEC & Sham & Region 2 & Vessel 3 & N/A & 5.4 \\
\hline 5 & Vehicle & No PEC & Sham & Region 3 & Vessel 1 & N/A & 5.8 \\
\hline 5 & Vehicle & No PEC & Sham & Region 3 & Vessel 2 & N/A & 6.0 \\
\hline 5 & Vehicle & No PEC & Sham & Region 3 & Vessel 3 & N/A & 6.0 \\
\hline 6 & Vehicle & No PEC & Sham & Region 1 & Vessel 1 & N/A & 4.9 \\
\hline 6 & Vehicle & No PEC & Sham & Region 1 & Vessel 2 & N/A & 4.5 \\
\hline 6 & Vehicle & No PEC & Sham & Region 1 & Vessel 3 & N/A & 4.8 \\
\hline 6 & Vehicle & No PEC & Sham & Region 2 & Vessel 1 & N/A & 6.1 \\
\hline 6 & Vehicle & No PEC & Sham & Region 2 & Vessel 2 & N/A & 4.5 \\
\hline 6 & Vehicle & No PEC & Sham & Region 2 & Vessel 3 & N/A & 4.4 \\
\hline 6 & Vehicle & No PEC & Sham & Region 3 & Vessel 1 & N/A & 5.3 \\
\hline 6 & Vehicle & No PEC & Sham & Region 3 & Vessel 2 & N/A & 5.0 \\
\hline 6 & Vehicle & No PEC & Sham & Region 3 & Vessel 3 & N/A & 4.1 \\
\hline 1 & Thrombin & No PEC & Sham & Region 1 & Vessel 1 & N/A & 5.8 \\
\hline
\end{tabular}




\begin{tabular}{|c|c|c|c|c|c|c|c|}
\hline 1 & Thrombin & No PEC & Sham & Region 1 & Vessel 2 & N/A & 4.8 \\
\hline 1 & Thrombin & No PEC & Sham & Region 1 & Vessel 3 & N/A & 4.4 \\
\hline 1 & Thrombin & No PEC & Sham & Region 2 & Vessel 1 & N/A & 6.6 \\
\hline 1 & Thrombin & No PEC & Sham & Region 2 & Vessel 2 & N/A & 6.3 \\
\hline 1 & Thrombin & No PEC & Sham & Region 2 & Vessel 3 & $\mathrm{~N} / \mathrm{A}$ & 5.0 \\
\hline 1 & Thrombin & No PEC & Sham & Region 3 & Vessel 1 & N/A & 4.4 \\
\hline 1 & Thrombin & No PEC & Sham & Region 3 & Vessel 2 & N/A & 4.9 \\
\hline 1 & Thrombin & No PEC & Sham & Region 3 & Vessel 3 & N/A & 4.9 \\
\hline 2 & Thrombin & PEC & Sham & Region 1 & Vessel 1 & N/A & 5.2 \\
\hline 2 & Thrombin & PEC & Sham & Region 1 & Vessel 2 & $\mathrm{~N} / \mathrm{A}$ & 5.2 \\
\hline 2 & Thrombin & PEC & Sham & Region 1 & Vessel 3 & $\mathrm{~N} / \mathrm{A}$ & 4.3 \\
\hline 2 & Thrombin & PEC & Sham & Region 2 & Vessel 1 & N/A & 3.9 \\
\hline 2 & Thrombin & PEC & Sham & Region 2 & Vessel 2 & N/A & 5.5 \\
\hline 2 & Thrombin & PEC & Sham & Region 2 & Vessel 3 & N/A & 4.4 \\
\hline 2 & Thrombin & PEC & Sham & Region 3 & Vessel 1 & N/A & 7.0 \\
\hline 2 & Thrombin & PEC & Sham & Region 3 & Vessel 2 & N/A & 7.1 \\
\hline 2 & Thrombin & PEC & Sham & Region 3 & Vessel 3 & N/A & 5.0 \\
\hline 3 & Thrombin & No PEC & Sham & Region 1 & Vessel 1 & N/A & 5.9 \\
\hline 3 & Thrombin & No PEC & Sham & Region 1 & Vessel 2 & N/A & 4.9 \\
\hline 3 & Thrombin & No PEC & Sham & Region 1 & Vessel 3 & N/A & 5.2 \\
\hline 3 & Thrombin & No PEC & Sham & Region 2 & Vessel 1 & N/A & 3.8 \\
\hline 3 & Thrombin & No PEC & Sham & Region 2 & Vessel 2 & N/A & 4.5 \\
\hline 3 & Thrombin & No PEC & Sham & Region 2 & Vessel 3 & N/A & 5.6 \\
\hline 3 & Thrombin & No PEC & Sham & Region 3 & Vessel 1 & N/A & 4.1 \\
\hline 3 & Thrombin & No PEC & Sham & Region 3 & Vessel 2 & N/A & 5.2 \\
\hline 3 & Thrombin & No PEC & Sham & Region 3 & Vessel 3 & N/A & 3.7 \\
\hline 4 & Thrombin & No PEC & Sham & Region 1 & Vessel 1 & N/A & 4.8 \\
\hline 4 & Thrombin & No PEC & Sham & Region 1 & Vessel 2 & N/A & 4.7 \\
\hline 4 & Thrombin & No PEC & Sham & Region 1 & Vessel 3 & N/A & 5.5 \\
\hline 4 & Thrombin & No PEC & Sham & Region 2 & Vessel 1 & N/A & 4.3 \\
\hline 4 & Thrombin & No PEC & Sham & Region 2 & Vessel 2 & N/A & 5.6 \\
\hline 4 & Thrombin & No PEC & Sham & Region 2 & Vessel 3 & N/A & 4.6 \\
\hline 4 & Thrombin & No PEC & Sham & Region 3 & Vessel 1 & N/A & 4.6 \\
\hline 4 & Thrombin & No PEC & Sham & Region 3 & Vessel 2 & N/A & 4.7 \\
\hline 4 & Thrombin & No PEC & Sham & Region 3 & Vessel 3 & N/A & 4.2 \\
\hline 5 & Thrombin & PEC & Sham & Region 1 & Vessel 1 & N/A & 5.2 \\
\hline 5 & Thrombin & PEC & Sham & Region 1 & Vessel 2 & N/A & 4.0 \\
\hline 5 & Thrombin & PEC & Sham & Region 1 & Vessel 3 & N/A & 5.0 \\
\hline 5 & Thrombin & PEC & Sham & Region 2 & Vessel 1 & N/A & 5.3 \\
\hline
\end{tabular}




\begin{tabular}{|c|c|c|c|c|c|c|c|}
\hline 5 & Thrombin & PEC & Sham & Region 2 & Vessel 2 & N/A & 3.7 \\
\hline 5 & Thrombin & PEC & Sham & Region 2 & Vessel 3 & N/A & 4.1 \\
\hline 5 & Thrombin & PEC & Sham & Region 3 & Vessel 1 & N/A & 4.7 \\
\hline 5 & Thrombin & PEC & Sham & Region 3 & Vessel 2 & N/A & 4.7 \\
\hline 5 & Thrombin & PEC & Sham & Region 3 & Vessel 3 & $\mathrm{~N} / \mathrm{A}$ & 4.4 \\
\hline 1 & Ligation Only & PEC & Sham & Region 1 & Vessel 1 & N/A & 5.2 \\
\hline 1 & Ligation Only & PEC & Sham & Region 1 & Vessel 2 & N/A & 5.0 \\
\hline 1 & Ligation Only & PEC & Sham & Region 1 & Vessel 3 & N/A & 4.6 \\
\hline 1 & Ligation Only & PEC & Sham & Region 2 & Vessel 1 & N/A & 6.0 \\
\hline 1 & Ligation Only & PEC & Sham & Region 2 & Vessel 2 & N/A & 6.6 \\
\hline 1 & Ligation Only & PEC & Sham & Region 2 & Vessel 3 & N/A & 4.9 \\
\hline 1 & Ligation Only & PEC & Sham & Region 3 & Vessel 1 & N/A & 4.9 \\
\hline 1 & Ligation Only & PEC & Sham & Region 3 & Vessel 2 & N/A & 4.2 \\
\hline 1 & Ligation Only & PEC & Sham & Region 3 & Vessel 3 & N/A & 6.2 \\
\hline 2 & Ligation Only & No PEC & Sham & Region 1 & Vessel 1 & N/A & 4.3 \\
\hline 2 & Ligation Only & No PEC & Sham & Region 1 & Vessel 2 & N/A & 5.3 \\
\hline 2 & Ligation Only & No PEC & Sham & Region 1 & Vessel 3 & N/A & 5.8 \\
\hline 2 & Ligation Only & No PEC & Sham & Region 2 & Vessel 1 & $\mathrm{~N} / \mathrm{A}$ & 5.7 \\
\hline 2 & Ligation Only & No PEC & Sham & Region 2 & Vessel 2 & N/A & 5.7 \\
\hline 2 & Ligation Only & No PEC & Sham & Region 2 & Vessel 3 & $\mathrm{~N} / \mathrm{A}$ & 5.0 \\
\hline 2 & Ligation Only & No PEC & Sham & Region 3 & Vessel 1 & N/A & 5.2 \\
\hline 2 & Ligation Only & No PEC & Sham & Region 3 & Vessel 2 & $\mathrm{~N} / \mathrm{A}$ & 6.7 \\
\hline 2 & Ligation Only & No PEC & Sham & Region 3 & Vessel 3 & N/A & 8.4 \\
\hline 3 & Ligation Only & No PEC & Sham & Region 1 & Vessel 1 & N/A & 3.9 \\
\hline 3 & Ligation Only & No PEC & Sham & Region 1 & Vessel 2 & N/A & 5.5 \\
\hline 3 & Ligation Only & No PEC & Sham & Region 1 & Vessel 3 & N/A & 4.8 \\
\hline 3 & Ligation Only & No PEC & Sham & Region 2 & Vessel 1 & N/A & 5.6 \\
\hline 3 & Ligation Only & No PEC & Sham & Region 2 & Vessel 2 & N/A & 5.7 \\
\hline 3 & Ligation Only & No PEC & Sham & Region 2 & Vessel 3 & N/A & 5.6 \\
\hline 3 & Ligation Only & No PEC & Sham & Region 3 & Vessel 1 & N/A & 5.4 \\
\hline 3 & Ligation Only & No PEC & Sham & Region 3 & Vessel 2 & $\mathrm{~N} / \mathrm{A}$ & 4.8 \\
\hline 3 & Ligation Only & No PEC & Sham & Region 3 & Vessel 3 & N/A & 6.1 \\
\hline 4 & Ligation Only & No PEC & Sham & Region 1 & Vessel 1 & N/A & 4.9 \\
\hline 4 & Ligation Only & No PEC & Sham & Region 1 & Vessel 2 & N/A & 5.2 \\
\hline 4 & Ligation Only & No PEC & Sham & Region 1 & Vessel 3 & N/A & 5.6 \\
\hline 4 & Ligation Only & No PEC & Sham & Region 2 & Vessel 1 & N/A & 4.9 \\
\hline 4 & Ligation Only & No PEC & Sham & Region 2 & Vessel 2 & $\mathrm{~N} / \mathrm{A}$ & 5.1 \\
\hline 4 & Ligation Only & No PEC & Sham & Region 2 & Vessel 3 & $\mathrm{~N} / \mathrm{A}$ & 7.1 \\
\hline 4 & Ligation Only & No PEC & Sham & Region 3 & Vessel 1 & N/A & 4.3 \\
\hline
\end{tabular}




\begin{tabular}{|c|c|c|c|c|c|c|c|}
\hline 4 & Ligation Only & No PEC & Sham & Region 3 & Vessel 2 & N/A & 5.9 \\
\hline 4 & Ligation Only & No PEC & Sham & Region 3 & Vessel 3 & N/A & 4.6 \\
\hline 5 & Ligation Only & PEC & Sham & Region 1 & Vessel 1 & N/A & 4.0 \\
\hline 5 & Ligation Only & PEC & Sham & Region 1 & Vessel 2 & N/A & 4.9 \\
\hline 5 & Ligation Only & PEC & Sham & Region 1 & Vessel 3 & N/A & 5.0 \\
\hline 5 & Ligation Only & PEC & Sham & Region 2 & Vessel 1 & N/A & 6.7 \\
\hline 5 & Ligation Only & PEC & Sham & Region 2 & Vessel 2 & N/A & 5.7 \\
\hline 5 & Ligation Only & PEC & Sham & Region 2 & Vessel 3 & N/A & 6.3 \\
\hline 5 & Ligation Only & PEC & Sham & Region 3 & Vessel 1 & N/A & 4.9 \\
\hline 5 & Ligation Only & PEC & Sham & Region 3 & Vessel 2 & N/A & 5.9 \\
\hline 5 & Ligation Only & PEC & Sham & Region 3 & Vessel 3 & N/A & 6.2 \\
\hline
\end{tabular}


Cell Transplantation Maximum Diameter Data

\begin{tabular}{|c|c|c|c|c|}
\hline Replicate & Treatment & Muscle & $\begin{array}{c}\text { Max } \alpha-S M A \\
\text { Vessel Diameter } \\
(\mu \mathrm{m})\end{array}$ & $\begin{array}{c}\text { Number of Vessels in } \\
\text { Region with Max } \\
\text { Diameter Vessel }\end{array}$ \\
\hline 1 & Myogenic Cells & Ligated & PEC & PEC \\
\hline 2 & Myogenic Cells & Ligated & 44.1 & 5 \\
\hline 3 & Myogenic Cells & Ligated & PEC & PEC \\
\hline 4 & Myogenic Cells & Ligated & 17.7 & 12 \\
\hline 5 & Myogenic Cells & Ligated & 28.7 & 8 \\
\hline 6 & Myogenic Cells & Ligated & 28.9 & 9 \\
\hline 1 & Vehicle & Ligated & PEC & PEC \\
\hline 2 & Vehicle & Ligated & PEC & PEC \\
\hline 3 & Vehicle & Ligated & 9.3 & 15 \\
\hline 4 & Vehicle & Ligated & 18.0 & 12 \\
\hline 5 & Vehicle & Ligated & 23.6 & 16 \\
\hline 6 & Vehicle & Ligated & 20.9 & 16 \\
\hline 1 & Thrombin & Ligated & 25.0 & 10 \\
\hline 2 & Thrombin & Ligated & PEC & PEC \\
\hline 3 & Thrombin & Ligated & 23.0 & 8 \\
\hline 4 & Thrombin & Ligated & 35.6 & 11 \\
\hline 5 & Thrombin & Ligated & PEC & PEC \\
\hline 1 & Ligation Only & Ligated & PEC & PEC \\
\hline 2 & Ligation Only & Ligated & 19.1 & 12 \\
\hline 3 & Ligation Only & Ligated & 15.2 & 15 \\
\hline 4 & Ligation Only & Ligated & 22.5 & 14 \\
\hline 5 & Ligation Only & Ligated & PEC & PEC \\
\hline
\end{tabular}


Cell Transplantation CD68 Data

\begin{tabular}{|c|c|c|c|c|c|}
\hline Replicate & Treatment & Muscle & Region & $\begin{array}{c}\text { Overall } \\
\text { CD68 Cell } \\
\text { Count } \\
\end{array}$ & $\begin{array}{c}\text { Vessel } \\
\text { Associated } \\
\text { CD68 Cells } \\
\end{array}$ \\
\hline 1 & Myogenic Cells & Ligated & Region 1 & 19 & 14 \\
\hline 1 & Myogenic Cells & Ligated & Region 2 & 15 & 11 \\
\hline 1 & Myogenic Cells & Ligated & Region 3 & 16 & 16 \\
\hline 2 & Myogenic Cells & Ligated & Region 1 & 15 & 21 \\
\hline 2 & Myogenic Cells & Ligated & Region 2 & 19 & 24 \\
\hline 2 & Myogenic Cells & Ligated & Region 3 & 16 & 15 \\
\hline 3 & Myogenic Cells & Ligated & Region 1 & 15 & 15 \\
\hline 3 & Myogenic Cells & Ligated & Region 2 & 14 & 13 \\
\hline 3 & Myogenic Cells & Ligated & Region 3 & 14 & 18 \\
\hline 4 & Myogenic Cells & Ligated & Region 1 & 14 & 20 \\
\hline 4 & Myogenic Cells & Ligated & Region 2 & 14 & 14 \\
\hline 4 & Myogenic Cells & Ligated & Region 3 & 15 & 20 \\
\hline 5 & Myogenic Cells & Ligated & Region 1 & 15 & 23 \\
\hline 5 & Myogenic Cells & Ligated & Region 2 & 20 & 16 \\
\hline 5 & Myogenic Cells & Ligated & Region 3 & 19 & 18 \\
\hline 6 & Myogenic Cells & Ligated & Region 1 & 16 & 16 \\
\hline 6 & Myogenic Cells & Ligated & Region 2 & 17 & 13 \\
\hline 6 & Myogenic Cells & Ligated & Region 3 & 15 & 18 \\
\hline 1 & Vehicle & Ligated & Region 1 & 15 & 16 \\
\hline 1 & Vehicle & Ligated & Region 2 & 15 & 16 \\
\hline 1 & Vehicle & Ligated & Region 3 & 16 & 17 \\
\hline 2 & Vehicle & Ligated & Region 1 & 16 & 14 \\
\hline 2 & Vehicle & Ligated & Region 2 & 14 & 16 \\
\hline 2 & Vehicle & Ligated & Region 3 & 15 & 12 \\
\hline 3 & Vehicle & Ligated & Region 1 & 18 & 14 \\
\hline 3 & Vehicle & Ligated & Region 2 & 21 & 20 \\
\hline 3 & Vehicle & Ligated & Region 3 & 15 & 12 \\
\hline 4 & Vehicle & Ligated & Region 1 & 14 & 12 \\
\hline 4 & Vehicle & Ligated & Region 2 & 17 & 13 \\
\hline 4 & Vehicle & Ligated & Region 3 & 17 & 13 \\
\hline 5 & Vehicle & Ligated & Region 1 & 16 & 17 \\
\hline 5 & Vehicle & Ligated & Region 2 & 15 & 19 \\
\hline 5 & Vehicle & Ligated & Region 3 & 17 & 11 \\
\hline 6 & Vehicle & Ligated & Region 1 & 14 & 18 \\
\hline 6 & Vehicle & Ligated & Region 2 & 14 & 11 \\
\hline
\end{tabular}




\begin{tabular}{|c|c|c|c|c|c|}
\hline 6 & Vehicle & Ligated & Region 3 & 15 & 12 \\
\hline 1 & Thrombin & Ligated & Region 1 & 19 & 14 \\
\hline 1 & Thrombin & Ligated & Region 2 & 16 & 15 \\
\hline 1 & Thrombin & Ligated & Region 3 & 17 & 15 \\
\hline 2 & Thrombin & Ligated & Region 1 & 15 & 16 \\
\hline 2 & Thrombin & Ligated & Region 2 & 14 & 15 \\
\hline 2 & Thrombin & Ligated & Region 3 & 16 & 15 \\
\hline 3 & Thrombin & Ligated & Region 1 & 10 & 12 \\
\hline 3 & Thrombin & Ligated & Region 2 & 16 & 20 \\
\hline 3 & Thrombin & Ligated & Region 3 & 16 & 16 \\
\hline 4 & Thrombin & Ligated & Region 1 & 16 & 19 \\
\hline 4 & Thrombin & Ligated & Region 2 & 16 & 19 \\
\hline 4 & Thrombin & Ligated & Region 3 & 16 & 22 \\
\hline 5 & Thrombin & Ligated & Region 1 & 14 & 13 \\
\hline 5 & Thrombin & Ligated & Region 2 & 15 & 17 \\
\hline 5 & Thrombin & Ligated & Region 3 & 14 & 13 \\
\hline 1 & Ligation Only & Ligated & Region 1 & 15 & 16 \\
\hline 1 & Ligation Only & Ligated & Region 2 & 18 & 15 \\
\hline 1 & Ligation Only & Ligated & Region 3 & 17 & 17 \\
\hline 2 & Ligation Only & Ligated & Region 1 & 19 & 20 \\
\hline 2 & Ligation Only & Ligated & Region 2 & 13 & 17 \\
\hline 2 & Ligation Only & Ligated & Region 3 & 17 & 18 \\
\hline 3 & Ligation Only & Ligated & Region 1 & 15 & 19 \\
\hline 3 & Ligation Only & Ligated & Region 2 & 15 & 22 \\
\hline 3 & Ligation Only & Ligated & Region 3 & 15 & 20 \\
\hline 4 & Ligation Only & Ligated & Region 1 & 13 & 19 \\
\hline 4 & Ligation Only & Ligated & Region 2 & 16 & 20 \\
\hline 4 & Ligation Only & Ligated & Region 3 & 13 & 16 \\
\hline 5 & Ligation Only & Ligated & Region 1 & 15 & 12 \\
\hline 5 & Ligation Only & Ligated & Region 2 & 15 & 12 \\
\hline 5 & Ligation Only & Ligated & Region 3 & 16 & 14 \\
\hline 1 & Myogenic Cells & Sham & Region 1 & 8 & N/A \\
\hline 1 & Myogenic Cells & Sham & Region 2 & 7 & N/A \\
\hline 1 & Myogenic Cells & Sham & Region 3 & 10 & $\mathrm{~N} / \mathrm{A}$ \\
\hline 2 & Myogenic Cells & Sham & Region 1 & 5 & $\mathrm{~N} / \mathrm{A}$ \\
\hline 2 & Myogenic Cells & Sham & Region 2 & 7 & N/A \\
\hline 2 & Myogenic Cells & Sham & Region 3 & 7 & $\mathrm{~N} / \mathrm{A}$ \\
\hline 3 & Myogenic Cells & Sham & Region 1 & 7 & N/A \\
\hline 3 & Myogenic Cells & Sham & Region 2 & 8 & $\mathrm{~N} / \mathrm{A}$ \\
\hline
\end{tabular}




\begin{tabular}{|c|c|c|c|c|c|}
\hline 3 & Myogenic Cells & Sham & Region 3 & 8 & $\mathrm{~N} / \mathrm{A}$ \\
\hline 4 & Myogenic Cells & Sham & Region 1 & 8 & $\mathrm{~N} / \mathrm{A}$ \\
\hline 4 & Myogenic Cells & Sham & Region 2 & 7 & $\mathrm{~N} / \mathrm{A}$ \\
\hline 4 & Myogenic Cells & Sham & Region 3 & 6 & $\mathrm{~N} / \mathrm{A}$ \\
\hline 5 & Myogenic Cells & Sham & Region 1 & 7 & $\mathrm{~N} / \mathrm{A}$ \\
\hline 5 & Myogenic Cells & Sham & Region 2 & 8 & $\mathrm{~N} / \mathrm{A}$ \\
\hline 5 & Myogenic Cells & Sham & Region 3 & 8 & $\mathrm{~N} / \mathrm{A}$ \\
\hline 6 & Myogenic Cells & Sham & Region 1 & 6 & N/A \\
\hline 6 & Myogenic Cells & Sham & Region 2 & 6 & $\mathrm{~N} / \mathrm{A}$ \\
\hline 6 & Myogenic Cells & Sham & Region 3 & 6 & $\mathrm{~N} / \mathrm{A}$ \\
\hline 1 & Vehicle & Sham & Region 1 & 7 & $\mathrm{~N} / \mathrm{A}$ \\
\hline 1 & Vehicle & Sham & Region 2 & 7 & $\mathrm{~N} / \mathrm{A}$ \\
\hline 1 & Vehicle & Sham & Region 3 & 6 & $\mathrm{~N} / \mathrm{A}$ \\
\hline 2 & Vehicle & Sham & Region 1 & 8 & $\mathrm{~N} / \mathrm{A}$ \\
\hline 2 & Vehicle & Sham & Region 2 & 11 & $\mathrm{~N} / \mathrm{A}$ \\
\hline 2 & Vehicle & Sham & Region 3 & 8 & $\mathrm{~N} / \mathrm{A}$ \\
\hline 3 & Vehicle & Sham & Region 1 & 9 & $\mathrm{~N} / \mathrm{A}$ \\
\hline 3 & Vehicle & Sham & Region 2 & 8 & $\mathrm{~N} / \mathrm{A}$ \\
\hline 3 & Vehicle & Sham & Region 3 & 6 & N/A \\
\hline 4 & Vehicle & Sham & Region 1 & 6 & $\mathrm{~N} / \mathrm{A}$ \\
\hline 4 & Vehicle & Sham & Region 2 & 7 & $\mathrm{~N} / \mathrm{A}$ \\
\hline 4 & Vehicle & Sham & Region 3 & 6 & $\mathrm{~N} / \mathrm{A}$ \\
\hline 5 & Vehicle & Sham & Region 1 & 6 & $\mathrm{~N} / \mathrm{A}$ \\
\hline 5 & Vehicle & Sham & Region 2 & 6 & $\mathrm{~N} / \mathrm{A}$ \\
\hline 5 & Vehicle & Sham & Region 3 & 6 & $\mathrm{~N} / \mathrm{A}$ \\
\hline 6 & Vehicle & Sham & Region 1 & 7 & N/A \\
\hline 6 & Vehicle & Sham & Region 2 & 6 & $\mathrm{~N} / \mathrm{A}$ \\
\hline 6 & Vehicle & Sham & Region 3 & 8 & $\mathrm{~N} / \mathrm{A}$ \\
\hline 1 & Thrombin & Sham & Region 1 & 7 & $\mathrm{~N} / \mathrm{A}$ \\
\hline 1 & Thrombin & Sham & Region 2 & 6 & N/A \\
\hline 1 & Thrombin & Sham & Region 3 & 7 & N/A \\
\hline 2 & Thrombin & Sham & Region 1 & 10 & N/A \\
\hline 2 & Thrombin & Sham & Region 2 & 9 & N/A \\
\hline 2 & Thrombin & Sham & Region 3 & 6 & N/A \\
\hline 3 & Thrombin & Sham & Region 1 & 6 & $\mathrm{~N} / \mathrm{A}$ \\
\hline 3 & Thrombin & Sham & Region 2 & 7 & $\mathrm{~N} / \mathrm{A}$ \\
\hline 3 & Thrombin & Sham & Region 3 & 8 & $\mathrm{~N} / \mathrm{A}$ \\
\hline 4 & Thrombin & Sham & Region 1 & 7 & $\mathrm{~N} / \mathrm{A}$ \\
\hline 4 & Thrombin & Sham & Region 2 & 6 & $\mathrm{~N} / \mathrm{A}$ \\
\hline
\end{tabular}




\begin{tabular}{|l|c|c|c|c|c|}
\hline 4 & Thrombin & Sham & Region 3 & 7 & N/A \\
\hline 5 & Thrombin & Sham & Region 1 & 6 & N/A \\
\hline 5 & Thrombin & Sham & Region 2 & 6 & N/A \\
\hline 5 & Thrombin & Sham & Region 3 & 7 & N/A \\
\hline 1 & Ligation Only & Sham & Region 1 & 8 & N/A \\
\hline 1 & Ligation Only & Sham & Region 2 & 9 & N/A \\
\hline 1 & Ligation Only & Sham & Region 3 & 8 & N/A \\
\hline 2 & Ligation Only & Sham & Region 1 & 10 & N/A \\
\hline 2 & Ligation Only & Sham & Region 2 & 6 & N/A \\
\hline 2 & Ligation Only & Sham & Region 3 & 5 & N/A \\
\hline 3 & Ligation Only & Sham & Region 1 & 5 & N/A \\
\hline 3 & Ligation Only & Sham & Region 2 & 6 & N/A \\
\hline 3 & Ligation Only & Sham & Region 3 & 5 & N/A \\
\hline 4 & Ligation Only & Sham & Region 1 & 7 & N/A \\
\hline 4 & Ligation Only & Sham & Region 2 & 7 & N/A \\
\hline 4 & Ligation Only & Sham & Region 3 & 7 & N/A \\
\hline 5 & Ligation Only & Sham & Region 1 & 7 & N/A \\
\hline 5 & Ligation Only & Sham & Region 2 & 8 & N/A \\
\hline 5 & Ligation Only & Sham & Region 3 & 7 & N/A \\
\hline
\end{tabular}




\section{Appendix D: Statistics}

Sample Independent t-test Outputs

Male versus Female $\alpha$-SMA vessel count

\section{Estimation for Difference \\ Difference $95 \% \mathrm{Cl}$ for Difference $0.3611 \quad(-0.0426,0.7648)$}

Test

Null hypothesis $\quad \mathrm{H}_{0}: \mu_{1}-\mu_{2}=0$

Alternative hypothesis $\mathrm{H}_{1}: \mu_{1}-\mu_{2} \neq 0$

\begin{tabular}{rrr} 
T-Value & DF & P-Value \\
\hline 1.85 & 24 & 0.0772
\end{tabular}

Male versus Female $\alpha$-SMA vessel diameter

Estimation for Difference

Difference $\quad 95 \% \mathrm{Cl}$ for Difference $0.4907 \quad(-0.5765,1.5580)$

Test

Null hypothesis $\quad \mathrm{H}_{0}: \mu_{1}-\mu_{2}=0$

Alternative hypothesis $H_{1}: \mu_{1}-\mu_{2} \neq 0$

\begin{tabular}{rrr} 
T-Value & DF & P-Value \\
\hline 0.92 & 63 & 0.3617
\end{tabular}


Sample One-Way ANOVAs with Tukey comparisons

\section{Cell Transplantation $\alpha$-SMA vessel counts}

Analysis of Variance

\begin{tabular}{lrrrrr} 
Source & DF & Adj SS & Adj MS & F-Value & P-Value \\
\hline Group & 3 & 61.458 & 20.4859 & 9.89 & $<0.0001$ \\
Error & 61 & 126.327 & 2.0709 & & \\
Total & 64 & 187.785 & & &
\end{tabular}

Model Summary

\begin{tabular}{rrrr} 
S & R-sq & R-sq(adj) & R-sq(pred) \\
\hline 1.43907 & $32.73 \%$ & $29.42 \%$ & $23.37 \%$
\end{tabular}

Means

\begin{tabular}{lrrrc} 
Group & $\mathrm{N}$ & Mean & StDev & $95 \% \mathrm{Cl}$ \\
\hline Cells & 18 & 7.1111 & 1.4907 & $(6.4329,7.7894)$ \\
Gel & 17 & 6.6471 & 0.6063 & $(5.9491,7.3450)$ \\
Ligation Only & 15 & 7.5333 & 1.3020 & $(6.7903,8.2763)$ \\
Thrombin & 15 & 9.2667 & 2.0517 & $(8.5237,10.0097)$ \\
Pooled StDev & $=1.43907$
\end{tabular}

Grouping Information Using the Tukey Method and 95\% Confidence

\begin{tabular}{lrrrr} 
Group & N & Mean & Grouping \\
\hline Thrombin & 15 & 9.2667 & A & \\
Ligation Only & 15 & 7.5333 & & B \\
Cells & 18 & 7.1111 & & B \\
Gel & 17 & 6.6471 & & B
\end{tabular}

Means that do not share a letter are significantly different.

Tukey Simultaneous Tests for Differences of Means

\begin{tabular}{lrrrrr} 
Difference of Levels & Difference of Means & SE of Difference & $95 \% \mathrm{Cl}$ & T-Value & Adjusted P-Value \\
\hline Gel-Cells & -0.4641 & 0.4867 & $(-1.7512,0.8230)$ & -0.95 & 0.7761 \\
Ligation Only-Cells & 0.4222 & 0.5031 & $(-0.9083,1.7527)$ & 0.84 & 0.8356 \\
Thrombin-Cells & 2.1556 & 0.5031 & $(0.8251,3.4861)$ & 4.28 & 0.0004 \\
Ligation Only-Gel & 0.8863 & 0.5098 & $(-0.4619,2.2344)$ & 1.74 & 0.3130 \\
Thrombin-Gel & 2.6196 & 0.5098 & $(1.2714,3.9678)$ & 5.14 & $<0.0001$ \\
Thrombin-Ligation Only & 1.7333 & 0.5255 & $(0.3437,3.1230)$ & 3.30 & 0.0086
\end{tabular}

Individual confidence level $=98.96 \%$
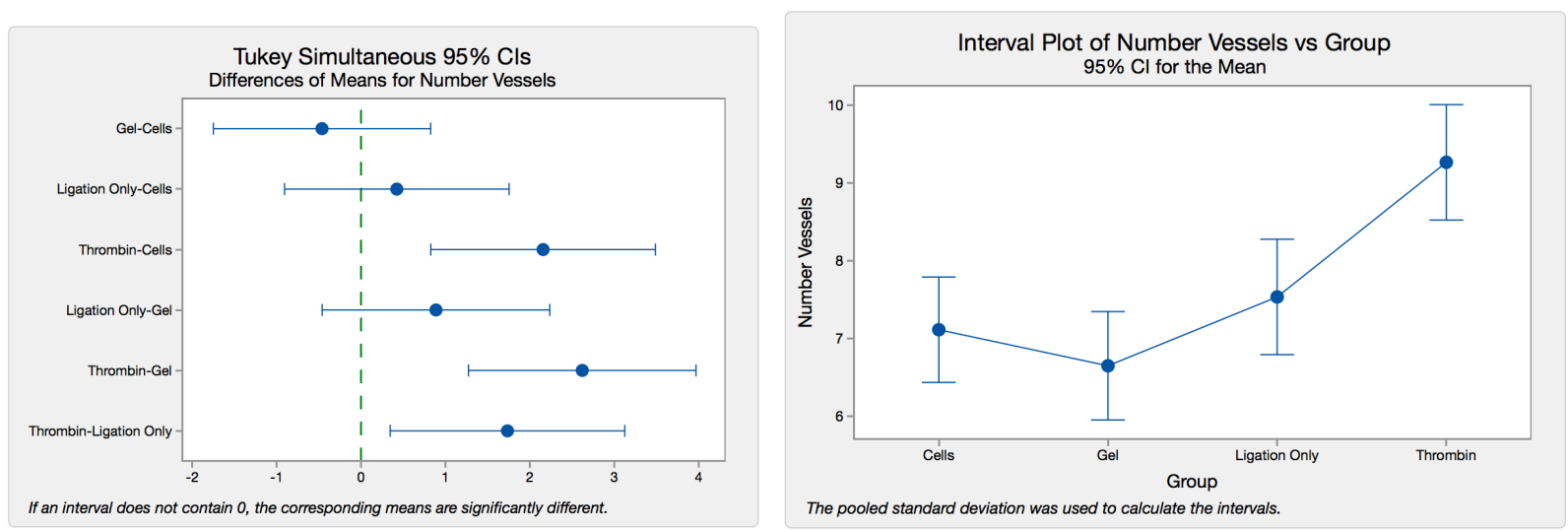


\section{Cell Transplantation Sham Vessel Count}

Analysis of Variance

\begin{tabular}{lrrrrr} 
Source & DF & Adj SS & Adj MS & F-Value & P-Value \\
\hline Group & 3 & 34.307 & 11.4357 & 2.09 & 0.1102 \\
Error & 62 & 338.678 & 5.4625 & & \\
Total & 65 & 372.985 & & &
\end{tabular}

Model Summary

\begin{tabular}{rrrr} 
S & R-sq & R-sq(adj) & R-sq(pred) \\
\hline 2.33721 & $9.20 \%$ & $4.80 \%$ & $0.00 \%$
\end{tabular}

\section{Means}

\begin{tabular}{lrrrc} 
Group & $\mathrm{N}$ & Mean & StDev & $95 \% \mathrm{Cl}$ \\
\hline Cells & 18 & 14.6667 & 2.1693 & $(13.5655,15.7679)$ \\
Gel & 18 & 12.7222 & 2.6078 & $(11.6210,13.8234)$ \\
Ligated Only & 15 & 13.6667 & 2.0587 & $(12.4604,14.8730)$ \\
Thrombin & 15 & 13.5333 & 2.4456 & $(12.3270,14.7396)$
\end{tabular}

Pooled StDev $=2.33721$

Grouping Information Using the Tukey Method and 95\% Confidence

\begin{tabular}{lrrl} 
Group & N & Mean & Grouping \\
\hline Cells & 18 & 14.6667 & A \\
Ligated Only & 15 & 13.6667 & A \\
Thrombin & 15 & 13.5333 & A \\
Gel & 18 & 12.7222 & A
\end{tabular}

Means that do not share a letter are significantly different.

Tukey Simultaneous Tests for Differences of Means

\begin{tabular}{lrrrrr} 
Difference of Levels & Difference of Means & SE of Difference & $95 \% \mathrm{Cl}$ & T-Value & Adjusted P-Value \\
\hline Gel-Cells & -1.9444 & 0.7791 & $(-3.9992,0.1104)$ & -2.50 & 0.0705 \\
Ligated Only-Cells & -1.0000 & 0.8171 & $(-3.1551,1.1551)$ & -1.22 & 0.6142 \\
Thrombin-Cells & -1.1333 & 0.8171 & $(-3.2884,1.0218)$ & -1.39 & 0.5122 \\
Ligated Only-Gel & 0.9444 & 0.8171 & $(-1.2107,3.0995)$ & 1.16 & 0.6566 \\
Thrombin-Gel & 0.8111 & 0.8171 & $(-1.3440,2.9662)$ & 0.99 & 0.7541 \\
Thrombin-Ligated Only & -0.1333 & 0.8534 & $(-2.3843,2.1176)$ & -0.16 & 0.9986
\end{tabular}

Individual confidence level $=98.95 \%$
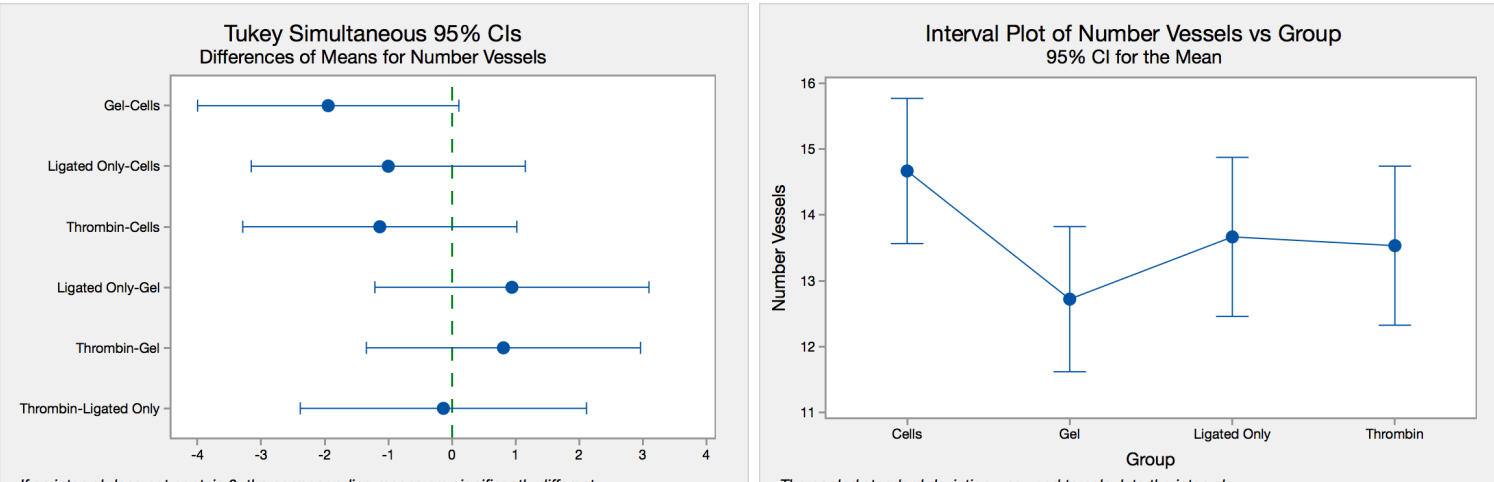

If an interval does not contain 0 , the corresponding means are significantly different.

The pooled standard deviation was used to calculate the intervals. 


\section{Cell Transplantation Total Vessel Count}

Analysis of Variance

\begin{tabular}{lrrrrr} 
Source & DF & Adj SS & Adj MS & F-Value & P-Value \\
\hline Group & 4 & 35.274 & 8.81843 & 1.84 & 0.1252 \\
Error & 127 & 608.696 & 4.79288 & & \\
Total & 131 & 643.970 & & &
\end{tabular}

Model Summary

\begin{tabular}{rrrr} 
S & R-sq & R-sq(adj) & R-sq(pred) \\
\hline 2.18927 & $5.48 \%$ & $2.50 \%$ & $0.00 \%$
\end{tabular}

Means

\begin{tabular}{lrrrc} 
Group & $\mathrm{N}$ & Mean & StDev & $95 \% \mathrm{Cl}$ \\
\hline Cells & 18 & 13.6111 & 2.1731 & $(12.5900,14.6322)$ \\
Gel & 18 & 12.5000 & 1.2005 & $(11.4789,13.5211)$ \\
Ligation Only & 15 & 13.6667 & 2.1602 & $(12.5481,14.7852)$ \\
Sham & 66 & 13.6515 & 2.3955 & $(13.1183,14.1848)$ \\
Thrombin & 15 & 12.4000 & 2.1647 & $(11.2814,13.5186)$
\end{tabular}

Pooled StDev $=2.18927$

Grouping Information Using the Tukey Method and 95\% Confidence

\begin{tabular}{lrrl} 
Group & N & Mean & Grouping \\
\hline Ligation Only & 15 & 13.6667 & A \\
Sham & 66 & 13.6515 & $\mathrm{~A}$ \\
Cells & 18 & 13.6111 & $\mathrm{~A}$ \\
Gel & 18 & 12.5000 & $\mathrm{~A}$ \\
Thrombin & 15 & 12.4000 & $\mathrm{~A}$
\end{tabular}

Means that do not share a letter are significantly different.

Tukey Simultaneous Tests for Differences of Means

\begin{tabular}{lrrrrr} 
Difference of Levels & Difference of Means & SE of Difference & $95 \% \mathrm{Cl}$ & T-Value & Adjusted P-Value \\
\hline Gel-Cells & -1.1111 & 0.7298 & $(-3.1287,0.9065)$ & -1.52 & 0.5498 \\
Ligation Only-Cells & 0.0556 & 0.7654 & $(-2.0605,2.1717)$ & 0.07 & 1.0000 \\
Sham-Cells & 0.0404 & 0.5821 & $(-1.5691,1.6499)$ & 0.07 & 1.0000 \\
Thrombin-Cells & -1.2111 & 0.7654 & $(-3.3272,0.9050)$ & -1.58 & 0.5114 \\
Ligation Only-Gel & 1.1667 & 0.7654 & $(-0.9494,3.2828)$ & 1.52 & 0.5487 \\
Sham-Gel & 1.1515 & 0.5821 & $(-0.4580,2.7610)$ & 1.98 & 0.2827 \\
Thrombin-Gel & -0.1000 & 0.7654 & $(-2.2161,2.0161)$ & -0.13 & 0.9999 \\
Sham-Ligation Only & -0.0152 & 0.6262 & $(-1.7465,1.7162)$ & -0.02 & 1.0000 \\
Thrombin-Ligation Only & -1.2667 & 0.7994 & $(-3.4769,0.9435)$ & -1.58 & 0.5101 \\
Thrombin-Sham & -1.2515 & 0.6262 & $(-2.9829,0.4798)$ & -2.00 & 0.2727
\end{tabular}

Individual confidence level $=99.35 \%$

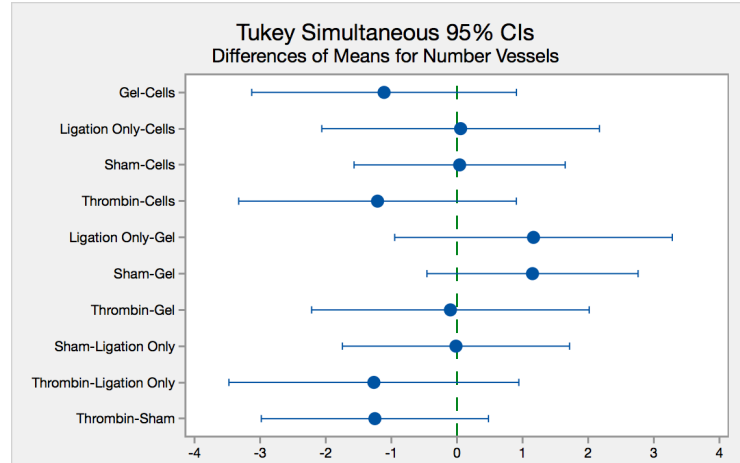

If an interval does not contain 0 , the corresponding means are significantly different.

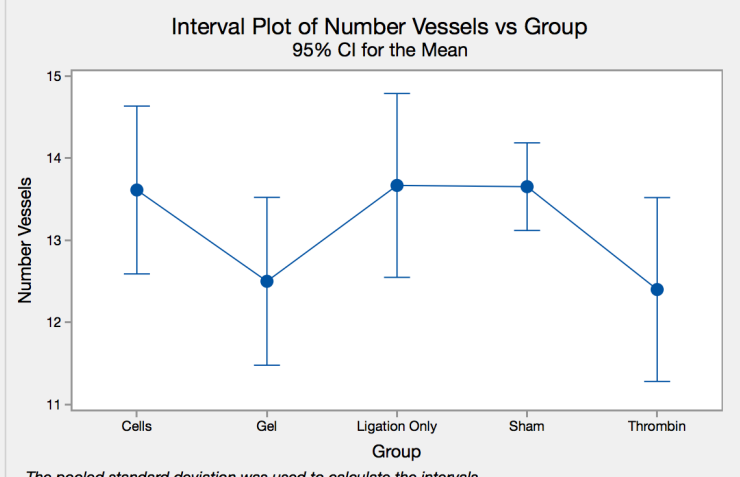

The pooled standard deviation was used to calculate the intervals. 


\section{Cell Transplantation $\alpha$-SMA Vessel Diameters}

Analysis of Variance

\begin{tabular}{lrrrrr} 
Source & DF & Adj SS & Adj MS & F-Value & P-Value \\
\hline Group & 3 & 373.97 & 124.655 & 6.87 & 0.0002 \\
Error & 191 & 3465.09 & 18.142 & & \\
Total & 194 & 3839.06 & & &
\end{tabular}

Model Summary

\begin{tabular}{rrrr} 
S & R-sq & R-sq(adj) & R-sq(pred) \\
\hline 4.25932 & $9.74 \%$ & $8.32 \%$ & $6.07 \%$
\end{tabular}

Means

\begin{tabular}{lrrrc} 
Group & N & Mean & StDev & $95 \% \mathrm{Cl}$ \\
\hline Cells & 54 & 13.4971 & 6.2256 & $(12.3538,14.6403)$ \\
Gel & 51 & 10.0868 & 2.7971 & $(8.9104,11.2632)$ \\
Ligation Only & 45 & 10.4503 & 3.2423 & $(9.1979,11.7027)$ \\
Thrombin & 45 & 11.9054 & 3.5585 & $(10.6530,13.1578)$ \\
Pooled StDev & $=4.25932$
\end{tabular}

Grouping Information Using the Tukey Method and 95\% Confidence

\begin{tabular}{lrrcc} 
Group & N & Mean & Grouping \\
\hline Cells & 54 & 13.4971 & A & \\
Thrombin & 45 & 11.9054 & A & B \\
Ligation Only & 45 & 10.4503 & & B \\
Gel & 51 & 10.0868 & & B
\end{tabular}

Means that do not share a letter are significantly different.

Tukey Simultaneous Tests for Differences of Means

\begin{tabular}{lrrrrr} 
Difference of Levels & Difference of Means & SE of Difference & $95 \%$ Cl & T-Value & Adjusted P-Value \\
\hline Gel-Cells & -3.4103 & 0.8317 & $(-5.5686,-1.2520)$ & -4.10 & 0.0004 \\
Ligation Only-Cells & -3.0467 & 0.8597 & $(-5.2778,-0.8157)$ & -3.54 & 0.0028 \\
Thrombin-Cells & -1.5917 & 0.8597 & $(-3.8227,0.6394)$ & -1.85 & 0.2528 \\
Ligation Only-Gel & 0.3636 & 0.8711 & $(-1.8971,2.6242)$ & 0.42 & 0.9755 \\
Thrombin-Gel & 1.8186 & 0.8711 & $(-0.4420,4.0793)$ & 2.09 & 0.1608 \\
Thrombin-Ligation Only & 1.4551 & 0.8979 & $(-0.8752,3.7853)$ & 1.62 & 0.3696
\end{tabular}

Individual confidence level $=98.98 \%$
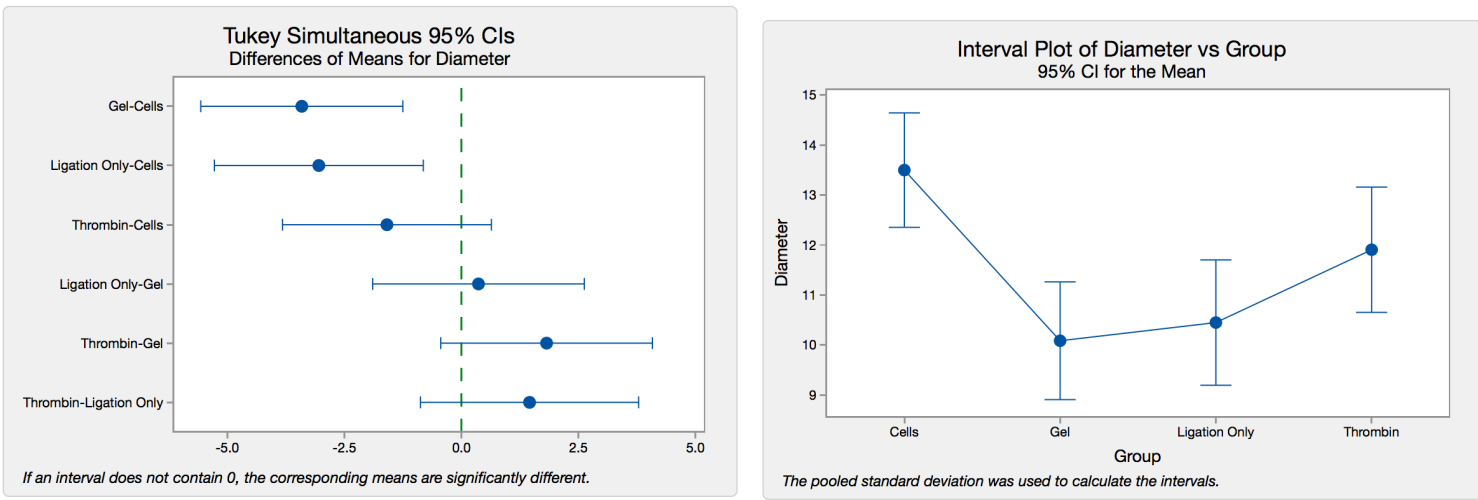


\section{Cell Transplantation Mice without a PEC Vessel Count}

Analysis of Variance

\begin{tabular}{lrrrrr} 
Source & DF & Adj SS & Adj MS & F-Value & P-Value \\
\hline Group & 3 & 56.258 & 18.7527 & 14.33 & $<0.0001$ \\
Error & 34 & 44.505 & 1.3090 & & \\
Total & 37 & 100.763 & & &
\end{tabular}

Model Summary

\begin{tabular}{rrrr} 
S & R-sq & R-sq(adj) & R-sq(pred) \\
\hline 1.14410 & $55.83 \%$ & $51.93 \%$ & $44.36 \%$
\end{tabular}

Means

\begin{tabular}{lrrrc} 
Group & $\mathrm{N}$ & Mean & StDev & $95 \% \mathrm{Cl}$ \\
\hline Cells & 9 & 7.8889 & 0.9280 & $(7.1139,8.6639)$ \\
Gel & 11 & 6.5455 & 0.6876 & $(5.8444,7.2465)$ \\
Ligation Only & 9 & 7.6667 & 1.5000 & $(6.8916,8.4417)$ \\
Thrombin & 9 & 9.8889 & 1.3642 & $(9.1139,10.6639)$
\end{tabular}

Pooled StDev $=1.14410$

Grouping Information Using the Tukey Method and 95\% Confidence

\begin{tabular}{|c|c|c|c|}
\hline Group & $\mathrm{N}$ & Mean & Grouping \\
\hline Thrombin & 9 & 9.8889 & A \\
\hline Cells & 9 & 7.8889 & B \\
\hline Ligation Only & 9 & 7.6667 & B \\
\hline Gel & 11 & 6.5455 & B \\
\hline
\end{tabular}

Means that do not share a letter are significantly different.

Tukey Simultaneous Tests for Differences of Means

\begin{tabular}{lrrrrr} 
Difference of Levels & Difference of Means & SE of Difference & $95 \% \mathrm{Cl}$ & T-Value & Adjusted P-Value \\
\hline Gel-Cells & -1.3434 & 0.5142 & $(-2.7325,0.0456)$ & -2.61 & 0.0610 \\
Ligation Only-Cells & -0.2222 & 0.5393 & $(-1.6790,1.2346)$ & -0.41 & 0.9760 \\
Thrombin-Cells & 2.0000 & 0.5393 & $(0.5432,3.4568)$ & 3.71 & 0.0040 \\
Ligation Only-Gel & 1.1212 & 0.5142 & $(-0.2678,2.5102)$ & 2.18 & 0.1493 \\
Thrombin-Gel & 3.3434 & 0.5142 & $(1.9544,4.7325)$ & 6.50 & $<0.0001$ \\
Thrombin-Ligation Only & 2.2222 & 0.5393 & $(0.7654,3.6790)$ & 4.12 & 0.0013
\end{tabular}

Individual confidence level $=98.93 \%$
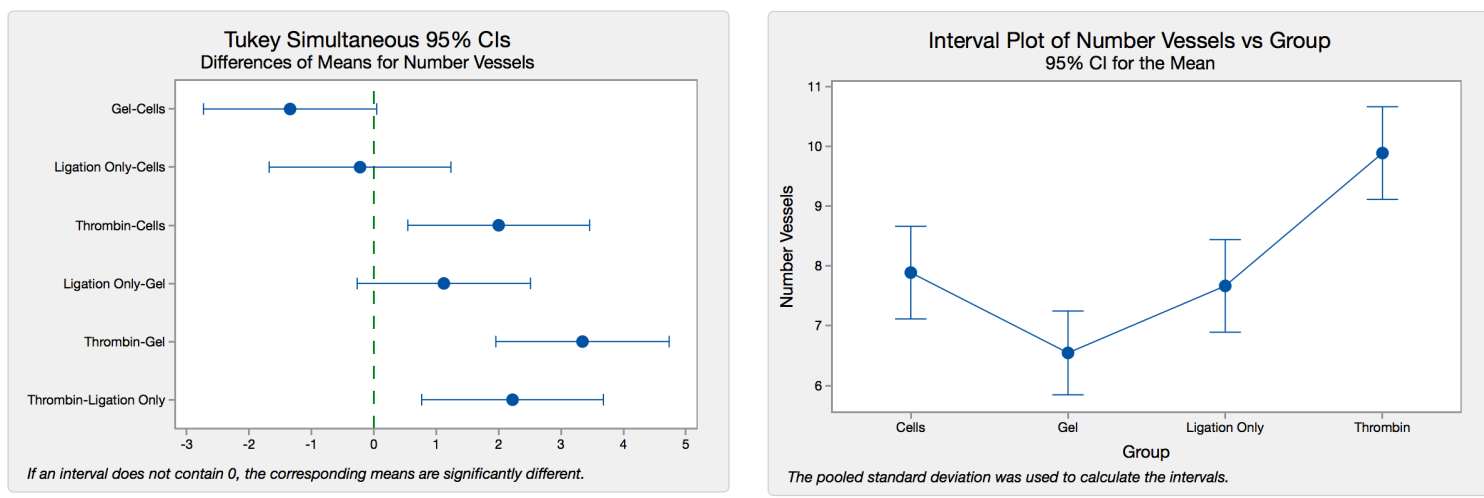
Cell Transplantation Vessel Count in the Region with the Maximum Diameter ACC

Analysis of Variance

\begin{tabular}{lrrrrr} 
Source & DF & Adj SS & Adj MS & F-Value & P-Value \\
\hline Group & 3 & 78.019 & 26.0064 & 9.46 & 0.0038 \\
Error & 9 & 24.750 & 2.7500 & & \\
Total & 12 & 102.769 & & &
\end{tabular}

Model Summary

\begin{tabular}{rrrr} 
S & R-sq & R-sq(adj) & R-sq(pred) \\
\hline 1.65831 & $75.92 \%$ & $67.89 \%$ & $50.75 \%$
\end{tabular}

Means

\begin{tabular}{lrrrc} 
Group & N & Mean & StDev & $95 \% \mathrm{Cl}$ \\
\hline Cells & 3 & 9.667 & 2.082 & $(7.501,11.833)$ \\
Gel & 4 & 14.7500 & 1.8930 & $(12.8743,16.6257)$ \\
Ligation Only & 3 & 14.3333 & 0.5774 & $(12.1675,16.4992)$ \\
Thrombin & 3 & 9.6667 & 1.5275 & $(7.5008,11.8325)$ \\
Pooled StDev & $=1.65831$ & &
\end{tabular}

Pooled StDev $=1.65831$

Grouping Information Using the Tukey Method and 95\% Confidence

\begin{tabular}{lrrrr} 
Group & N & Mean & Grouping \\
\hline Gel & 4 & 14.7500 & A & \\
Ligation Only & 3 & 14.3333 & A & \\
Thrombin & 3 & 9.6667 & & B \\
Cells & 3 & 9.667 & & B
\end{tabular}

Means that do not share a letter are significantly different.

Tukey Simultaneous Tests for Differences of Means

\begin{tabular}{lrrrrr} 
Difference of Levels & Difference of Means & SE of Difference & $95 \% \mathrm{Cl}$ & T-Value & Adjusted P-Value \\
\hline Gel-Cells & 5.083 & 1.267 & $(1.125,9.042)$ & 4.01 & 0.0133 \\
Ligation Only-Cells & 4.667 & 1.354 & $(0.435,8.898)$ & 3.45 & 0.0307 \\
Thrombin-Cells & 0.000 & 1.354 & $(-4.232,4.232)$ & 0.00 & 1.0000 \\
Ligation Only-Gel & -0.417 & 1.267 & $(-4.375,3.542)$ & -0.33 & 0.9869 \\
Thrombin-Gel & -5.083 & 1.267 & $(-9.042,-1.125)$ & -4.01 & 0.0133 \\
Thrombin-Ligation Only & -4.667 & 1.354 & $(-8.898,-0.435)$ & -3.45 & 0.0307
\end{tabular}

Individual confidence level $=98.78 \%$
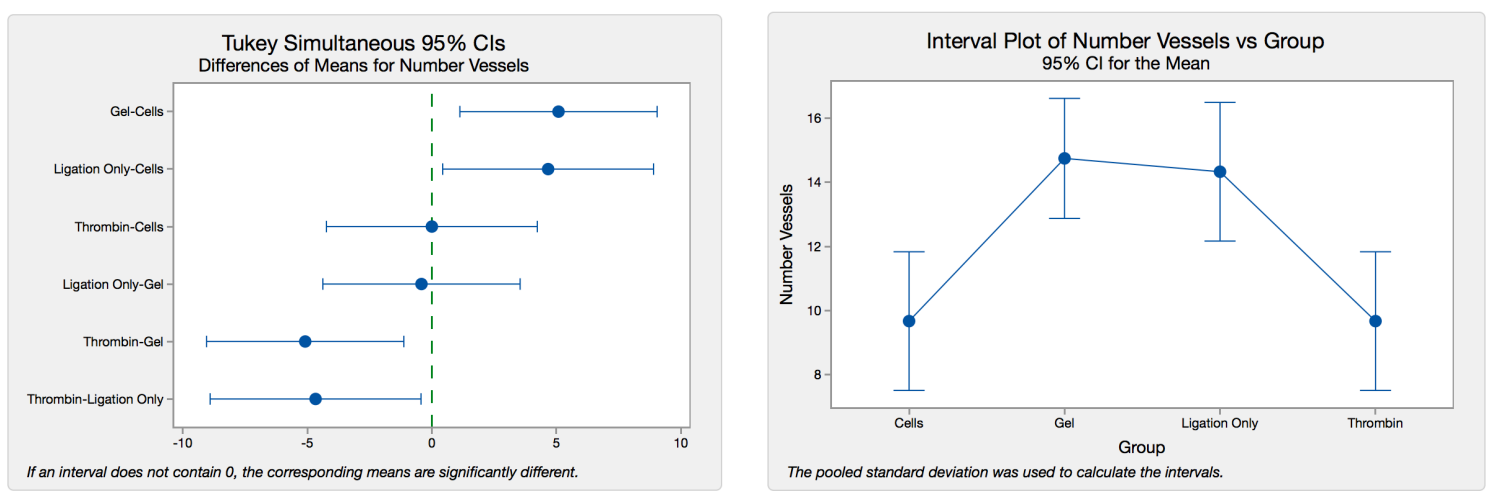


\section{Cell Transplantation Maximum Diameter Vessel}

Analysis of Variance

\begin{tabular}{lrrrrr} 
Source & DF & Adj SS & Adj MS & F-Value & P-Value \\
\hline Group & 3 & 1216.26 & 405.421 & 8.14 & 0.0003 \\
Error & 38 & 1892.38 & 49.799 & & \\
Total & 41 & 3108.64 & & &
\end{tabular}

Model Summary

\begin{tabular}{rrrr} 
S & R-sq & R-sq(adj) & R-sq(pred) \\
\hline 7.05687 & $39.13 \%$ & $34.32 \%$ & $26.64 \%$
\end{tabular}

\section{Means}

\begin{tabular}{lrrrc} 
Group & N & Mean & StDev & $95 \%$ Cl \\
\hline Cells & 12 & 29.854 & 10.204 & $(25.730,33.978)$ \\
Gelatin & 12 & 17.932 & 5.803 & $(13.808,22.056)$ \\
Ligation ONly & 9 & 18.906 & 3.336 & $(14.144,23.668)$ \\
Thrombin & 9 & 27.860 & 5.995 & $(23.098,32.622)$ \\
Pooled StDev $=7.05687$ & &
\end{tabular}

Grouping Information Using the Tukey Method and 95\% Confidence

\begin{tabular}{lrrrr} 
Group & N & Mean & Grouping \\
\hline Cells & 12 & 29.854 & A & \\
Thrombin & 9 & 27.860 & A & \\
Ligation ONly & 9 & 18.906 & & B \\
Gelatin & 12 & 17.932 & & B
\end{tabular}

Means that do not share a letter are significantly different.

Tukey Simultaneous Tests for Differences of Means

\begin{tabular}{lrrrrr} 
Difference of Levels & Difference of Means & SE of Difference & $95 \% \mathrm{Cl}$ & T-Value & Adjusted P-Value \\
\hline Gelatin-Cells & -11.922 & 2.881 & $(-19.663,-4.181)$ & -4.14 & 0.0010 \\
Ligation ONly-Cells & -10.948 & 3.112 & $(-19.309,-2.587)$ & -3.52 & 0.0060 \\
Thrombin-Cells & -1.994 & 3.112 & $(-10.355,6.368)$ & -0.64 & 0.9181 \\
Ligation ONly-Gelatin & 0.974 & 3.112 & $(-7.388,9.335)$ & 0.31 & 0.9892 \\
Thrombin-Gelatin & 9.928 & 3.112 & $(1.567,18.290)$ & 3.19 & 0.0145 \\
Thrombin-Ligation ONly & 8.954 & 3.327 & $(0.016,17.893)$ & 2.69 & 0.0494
\end{tabular}

Individual confidence level $=98.94 \%$
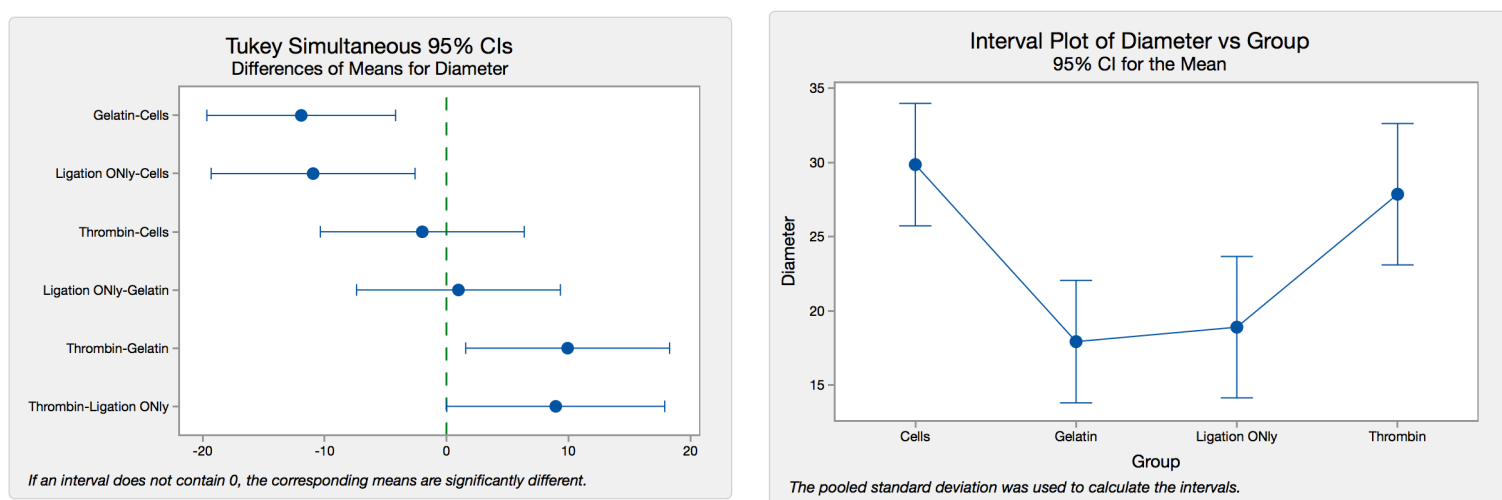


\section{Cell Transplantation Overall CD68)}

Analysis of Variance

\begin{tabular}{lrrrrr} 
Source & DF & Adj SS & Adj MS & F-Value & P-Value \\
\hline Group & 3 & 4.489 & 1.49630 & 0.43 & 0.7328 \\
Error & 62 & 216.178 & 3.48674 & & \\
Total & 65 & 220.667 & & &
\end{tabular}

Model Summary

\begin{tabular}{rrrr} 
S & R-sq & R-sq(adj) & R-sq(pred) \\
\hline 1.86728 & $2.03 \%$ & $0.00 \%$ & $0.00 \%$
\end{tabular}

\section{Means}

\begin{tabular}{lrrrc} 
Group & N & Mean & StDev & $95 \% \mathrm{Cl}$ \\
\hline Cells & 18 & 16.0000 & 1.9704 & $(15.1202,16.8798)$ \\
Gel & 18 & 15.7778 & 1.7675 & $(14.8980,16.6576)$ \\
Ligation Only & 15 & 15.4667 & 1.7674 & $(14.5029,16.4304)$ \\
Thrombin & 15 & 15.3333 & 1.9518 & $(14.3696,16.2971)$
\end{tabular}

Pooled StDev $=1.86728$

Grouping Information Using the Tukey Method and 95\% Confidence

\begin{tabular}{lrrl} 
Group & N & Mean & Grouping \\
\hline Cells & 18 & 16.0000 & A \\
Gel & 18 & 15.7778 & $\mathrm{~A}$ \\
Ligation Only & 15 & 15.4667 & $\mathrm{~A}$ \\
Thrombin & 15 & 15.3333 & $\mathrm{~A}$
\end{tabular}

Means that do not share a letter are significantly different.

Tukey Simultaneous Tests for Differences of Means

\begin{tabular}{lrrrrr} 
Difference of Levels & Difference of Means & SE of Difference & $95 \% \mathrm{Cl}$ & T-Value & Adjusted P-Value \\
\hline Gel-Cells & -0.2222 & 0.6224 & $(-1.8639,1.4194)$ & -0.36 & 0.9843 \\
Ligation Only-Cells & -0.5333 & 0.6528 & $(-2.2551,1.1885)$ & -0.82 & 0.8462 \\
Thrombin-Cells & -0.6667 & 0.6528 & $(-2.3885,1.0551)$ & -1.02 & 0.7377 \\
Ligation Only-Gel & -0.3111 & 0.6528 & $(-2.0329,1.4107)$ & -0.48 & 0.9640 \\
Thrombin-Gel & -0.4444 & 0.6528 & $(-2.1662,1.2773)$ & -0.68 & 0.9040 \\
Thrombin-Ligation Only & -0.1333 & 0.6818 & $(-1.9317,1.6650)$ & -0.20 & 0.9973
\end{tabular}

Individual confidence level $=98.95 \%$
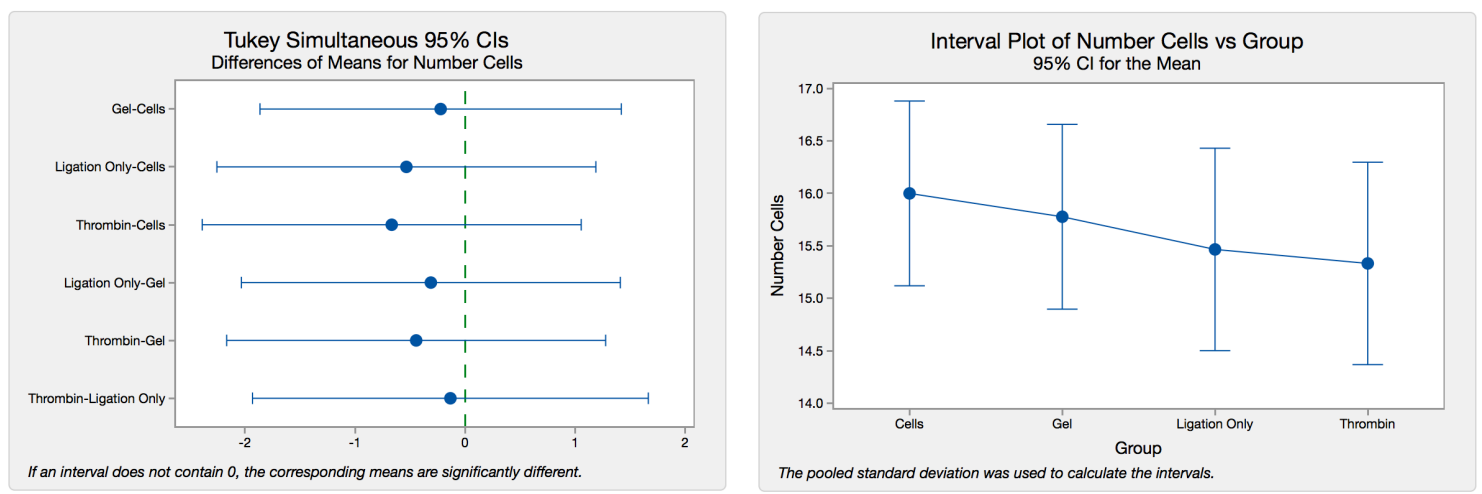


\section{Cell Transplantation Vessel Associated CD68 Cells}

Analysis of Variance

\begin{tabular}{lrrrrr} 
Source & DF & Adj SS & Adj MS & F-Value & P-Value \\
\hline Group & 3 & 68.596 & 22.8653 & 2.39 & 0.0775 \\
Error & 62 & 593.889 & 9.5789 & & \\
Total & 65 & 662.485 & & &
\end{tabular}

Model Summary

\begin{tabular}{rrrr} 
S & R-sq & R-sq(adj) & R-sq(pred) \\
\hline 3.09497 & $10.35 \%$ & $6.02 \%$ & $0.00 \%$
\end{tabular}

Means

\begin{tabular}{lrrrc} 
Group & $\mathrm{N}$ & Mean & StDev & $95 \% \mathrm{Cl}$ \\
\hline Cells & 18 & 16.9444 & 3.5887 & $(15.4862,18.4027)$ \\
Ligation & 15 & 17.1333 & 2.9968 & $(15.5359,18.7307)$ \\
Thrombin & 15 & 16.0667 & 2.8402 & $(14.4693,17.6641)$ \\
Vehicle & 18 & 14.6111 & 2.8313 & $(13.1529,16.0693)$
\end{tabular}

Pooled StDev $=3.09497$

Grouping Information Using the Tukey Method and 95\% Confidence

\begin{tabular}{lrrl} 
Group & $\mathrm{N}$ & Mean & Grouping \\
\hline Ligation & 15 & 17.1333 & $\mathrm{~A}$ \\
Cells & 18 & 16.9444 & $\mathrm{~A}$ \\
Thrombin & 15 & 16.0667 & $\mathrm{~A}$ \\
Vehicle & 18 & 14.6111 & $\mathrm{~A}$
\end{tabular}

Means that do not share a letter are significantly different.

Tukey Simultaneous Tests for Differences of Means

\begin{tabular}{lrrrrr} 
Difference of Levels & Difference of Means & SE of Difference & $95 \% \mathrm{Cl}$ & T-Value & Adjusted P-Value \\
\hline Ligation-Cells & 0.189 & 1.082 & $(-2.665,3.043)$ & 0.17 & 0.9981 \\
Thrombin-Cells & -0.878 & 1.082 & $(-3.732,1.976)$ & -0.81 & 0.8489 \\
Vehicle-Cells & -2.333 & 1.032 & $(-5.054,0.388)$ & -2.26 & 0.1184 \\
Thrombin-Ligation & -1.067 & 1.130 & $(-4.047,1.914)$ & -0.94 & 0.7814 \\
Vehicle-Ligation & -2.522 & 1.082 & $(-5.376,0.332)$ & -2.33 & 0.1020 \\
Vehicle-Thrombin & -1.456 & 1.082 & $(-4.309,1.398)$ & -1.35 & 0.5381
\end{tabular}

Individual confidence level $=98.95 \%$
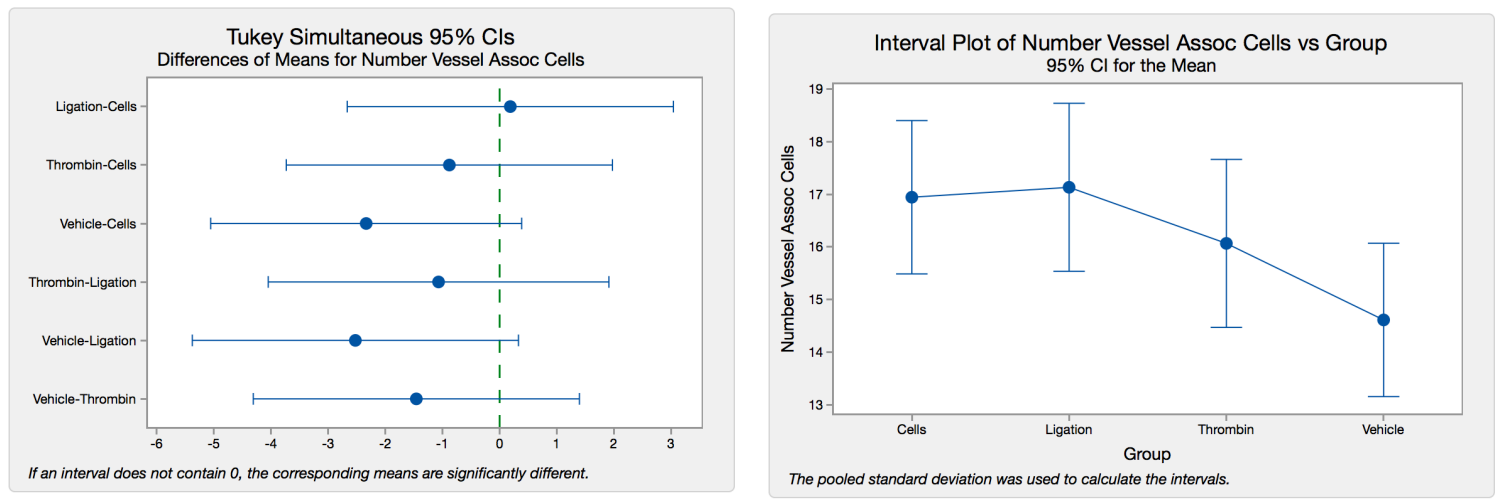
Mice with a PEC Sample Size Power Analysis Graph

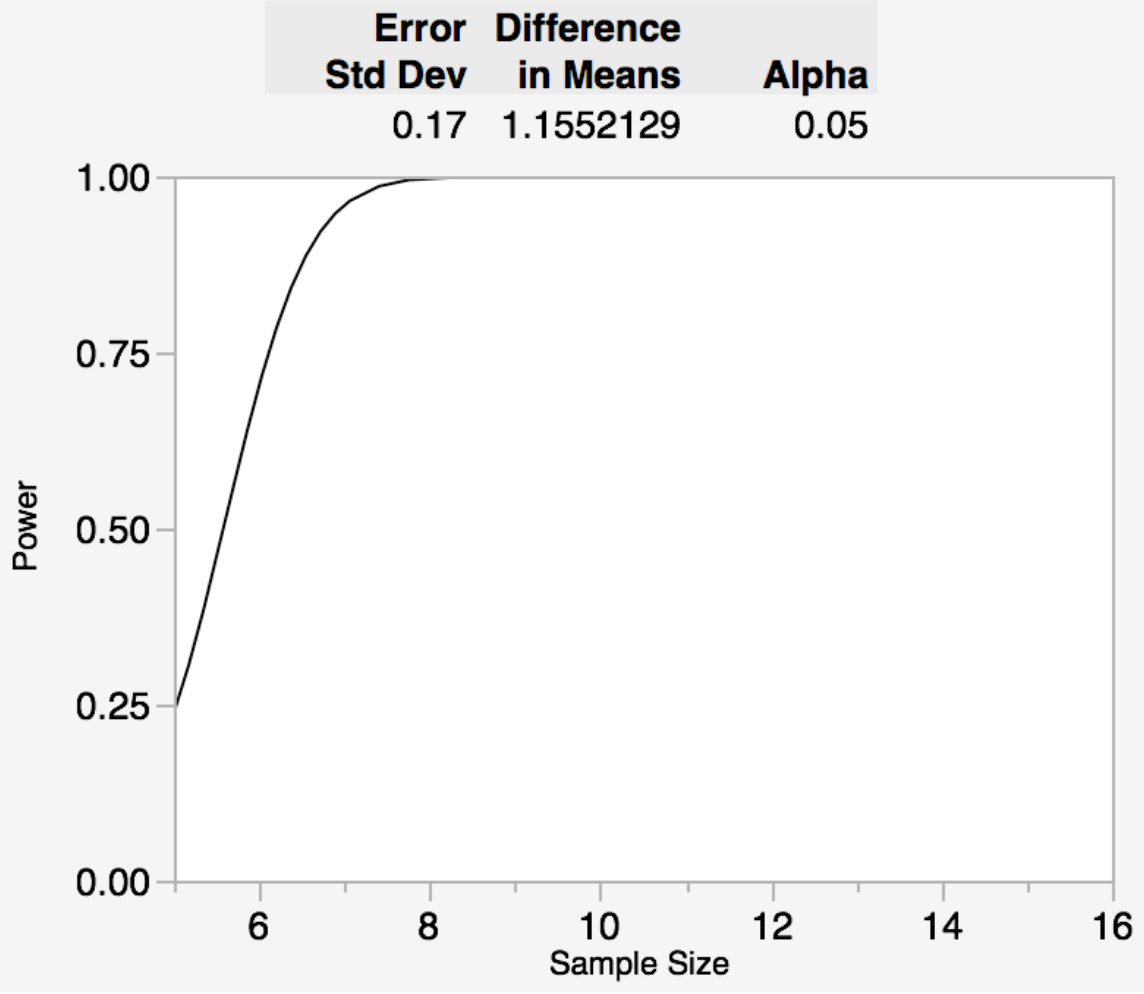

Mice without a PEC Sample Size Power Analysis Graph

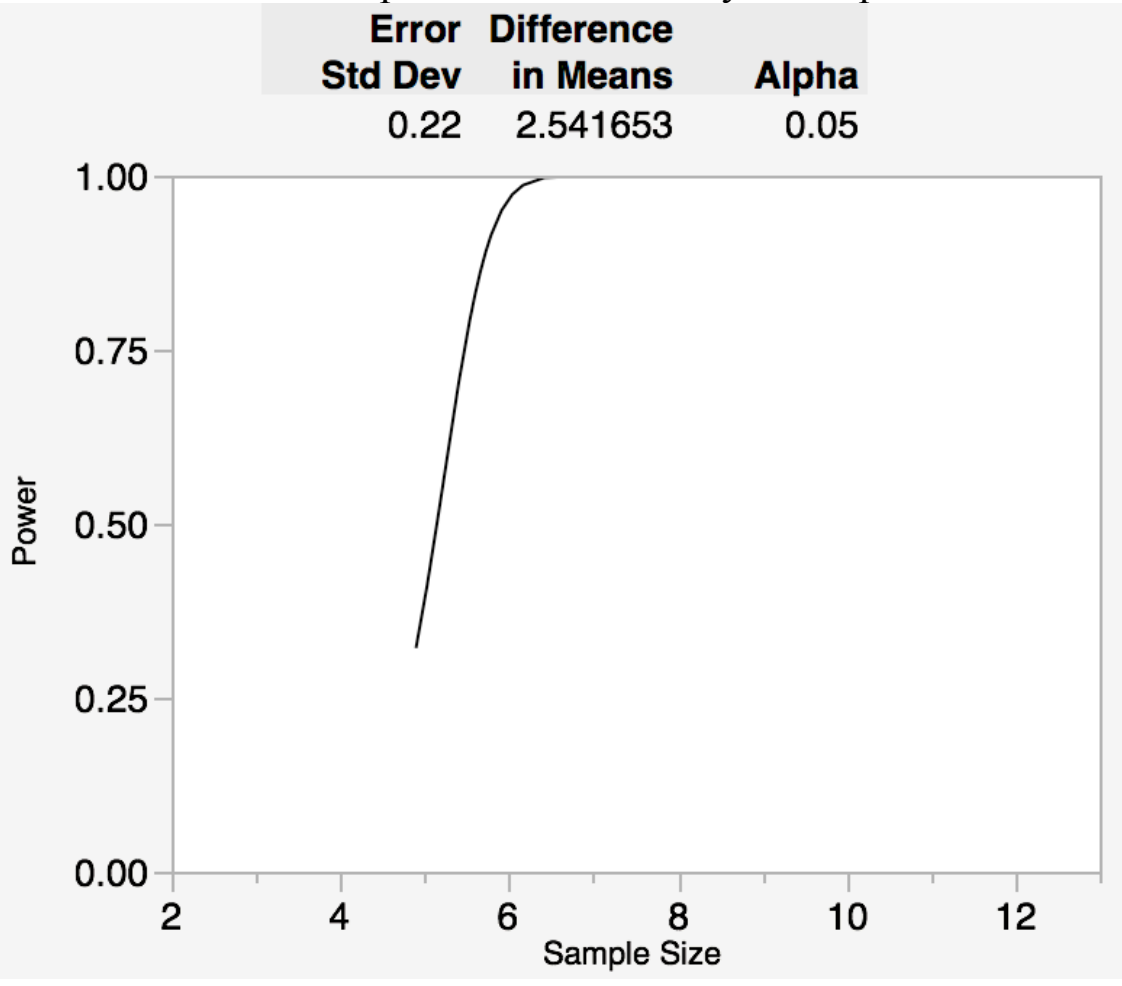

